\title{
Eigenfunctions and nodal sets
}

\author{
Steve Zelditch
}

\begin{abstract}
This is a survey of recent results on nodal sets of eigenfunctions of the Laplacian on Riemannian manifolds. The emphasis is on complex nodal sets of analytic continuations of eigenfunctions.
\end{abstract}

Let $(M, g)$ be a (usually compact) Riemannian manifold of dimension $n$, and let $\left\{\varphi_{j}\right\}$ denote an orthonormal basis of eigenfunctions of its Laplacian,

$$
\Delta_{g} \varphi_{j}=-\lambda_{j}^{2} \varphi_{j} \quad\left\langle\varphi_{j}, \varphi_{k}\right\rangle=\delta_{j k} .
$$

Here $\langle u, v\rangle=\int_{M} u v d V_{g}$ where $d V_{g}$ is the volume form of $(M, g)$. If $\partial M \neq 0$ we impose Dirichlet or Neumann boundary conditions. When $(M, g)$ is compact, the spectrum of $\Delta$ is discrete and can be put in non-decreasing order $\lambda_{0}<\lambda_{1} \leq \lambda_{2} \uparrow \infty$. The eigenvalues $\lambda_{j}^{2}$ are often termed energies while their square roots $\lambda_{j}$ are often termed the frequencies. The nodal set of an eigenfunction $\varphi_{\lambda}$ is the zero set

$$
Z_{\varphi_{\lambda}}{ }^{1}=\left\{x \in M: \varphi_{\lambda}(x)=0\right\} .
$$

The aim of this survey is to review some recent results on the $\mathcal{H}^{n-1}$-surface measure and on the yet more difficult problem of the spatial distribution of the nodal sets, i.e. the behavior of the integrals

$$
\frac{1}{\lambda_{j}} \int_{Z_{\varphi_{\lambda_{j}}}} f d S_{\lambda_{j}}, \quad(f \in C(M))
$$

as $\lambda \rightarrow \infty$. Here, $d S_{\lambda}=d \mathcal{H}^{n-1}$ denotes the Riemannian hypersurface volume form on $Z_{\varphi_{\lambda}}$. More generally, we consider the same problems for any level set

$$
\mathcal{N}_{\varphi_{\lambda}}^{c}:=\left\{\varphi_{\lambda}=c\right\}
$$

where $c$ is a constant (which in general may depend on $\lambda$ ). Nodal sets are special level sets and much more attention has been devoted to them than

Research partially supported by NSF grant \# DMS-0904252.

${ }^{1}$ In difference references we use either the notation $Z$ or $\mathcal{N}$ for the nodal set. Sometimes we use the subscript $\varphi_{\lambda}$ and sometimes only $\lambda$.

(C) 2013 International Press 
other level sets, but it is often of interest to study general level sets and in particular 'high level' sets or excursion sets.

We have recently written surveys $[\mathbf{Z 5}, \mathbf{Z 6}]$ on the global harmonic analysis of eigenfunctions, which include some discussion of nodal sets and critical point sets. To the extent possible, we hope to avoid repeating what is written there, but inevitably there will be some overlap. We refer there and $[\mathbf{H}]$ for background on well-established results. We also decided to cover some results of research in progress (especially from $[\mathbf{Z 3}]$, but also on $L^{\infty}$ quantum ergodic theory). We generally refer to the results as 'Conjectures' even when detailed arguments exist, since they have not yet been carefully examined by others.

There are two basic intuitions underlying many of the conjectures and results on eigenfunctions:

- Eigenfunctions of $\Delta_{g}$-eigenvalue $-\lambda^{2}$ are similar to polynomials of degree $\lambda$. In particular, $Z_{\lambda}$ is similar to a real algebraic variety of degree $\lambda$.

Of course, this intuition is most reliable when $(M, g)$ is real analytic. It is quite unclear at this time how reliable it is for general $C^{\infty}$ metrics, although there are some recent improvements on volumes and equidistribution in the smooth case.

- High frequency behavior of eigenfunctions reflects the dynamics of the geodesic flow $G^{t}: S^{*} M \rightarrow S^{*} M$ of $M$. Here, $S^{*} M$ is the unit co-sphere bundle of $(M, g)$.

When the dynamics is "chaotic" (highly ergodic), then eigenfunctions are de-localized and behave like Gaussian random waves of almost fixed frequency. This motivates the study of Gaussian random wave models for eigenfunctions, and suggests that in the 'chaotic case' nodal sets should be asympotically uniformly distributed.

When $G^{t}$ is completely integrable, model eigenfunctions are highly localized and their nodal sets are often exhibit quite regular patterns. The latter heuristic is not necessarily expected when there exist high multiplicities, as for rational flat tori, and then some weaker randomness can enter.

Both of these general intuitions lead to predictions about nodal sets and critical point sets. Most of the predictions are well beyond current or forseeable techniques to settle. A principal theme of this survey is that the analogues of such 'wild' predictions can sometimes be proved for real analytic $(M, g)$ if one analytically continues eigenfunctions to the complexification of $M$ and studies complex nodal sets instead of real ones.

As with algebraic varieties, nodal sets in the real analytic case are better behaved in the complex domain than the real domain. That is, zero sets of analytic continuations of eigenfunctions to the complexification of $M$ behave 
like complex algebraic varieties and also reflect the dynamics of the geodesic flow.

It is well-known that the complexification of $M$ can be identified with a neighborhood of the zero-section of the phase space $T^{*} M$. That is one reason why dynamics of the geodesic flow has greater impact on the complex nodal set.

We will exhibit a number of relatively recent results (some unpublished elsewhere) which justify this viewpoint:

- Theorem 8.4, which shows that complex methods can be used to give upper bounds on the number of nodal components of Dirichlet or Neumann eigenfunctions which "touch the boundary" of a real analytic plane domain.

- Theorem 9.1 on the limit distribution of the normalized currents of integration

$$
\frac{1}{\lambda_{j_{k}}}\left[Z_{\varphi_{j_{k}}^{\mathbb{C}}}\right]
$$

over the complex zero sets of "ergodic eigenfunctions" in the complex domain.

- Theorem 11.2 and Corollary 11.1, which show that the similar currents for analytic continuations of "Riemannian random waves" tend to the same limit almost surely. Thus, the prediction that zero sets of ergodic eigenfunctions agrees with that of random waves is correct in the complex domain.

- Sharper results on the distribution of intersections points of nodal sets and geodesics on complexified real analytic surfaces (Theorem 10.1).

Our analysis of nodal sets in the complex domain is based on the use of complex Fourier integral techniques (i.e. generalized Paley-Wiener theory). The principal tools are the analytic continuation of the Poissonwave kernel and the Szegö kernel in the complex domain. They become Fourier integral operators with complex phase and with wave fronts along the complexified geodesic flow. One can read off the growth properties of complexified eigenfunctions from mapping properties of such operators. Log moduli of complexified spectral projectors are asymptotically extremal plurisubharmonic functions for all $(M, g)$. These ideas are the basis of the articles $[\mathbf{Z 2}, \mathbf{T Z}, \mathbf{Z 3}, \mathbf{Z 4}, \mathbf{Z 8}, \mathbf{Z 9}, \mathbf{H e}]$. Such ideas have antecedents in work of S. Bernstein, Baouendi- Goulaouic, and Donnelly-Fefferman, Guillemin, F.H. Lin (among others) .

We note that the focus on complex nodal sets only makes sense for real analytic $(M, g)$. It is possible that one can study "almost analytic extensions" of eigenfunctions for general $C^{\infty}$ metrics in a similar spirit, but this is just a speculation and certain key methods break down when $g$ is not real analytic. Hence the results in the $C^{\infty}$ case are much less precise than in the real analytic case. 
It should also be mentioned that much work on eigenfunctions concerns ground states, i.e. the first and second eigenfunctions. Unfortunately, we do not have the space or expertise to review the results on ground states in this survey. For a sample we refer to [Me]. Further, many if not all of the techniques and results surveyed here have generalizations to Schrödinger operators $-\hbar^{2} \Delta+V$. For the sake of brevity we confine the discussion to the Laplacian.

0.1. Notation. The first notational issue is whether to choose $\Delta_{g}$ to be the positive or negative Laplacian. The traditional choice

$$
\Delta_{g}=\frac{1}{\sqrt{g}} \sum_{i, j=1}^{n} \frac{\partial}{\partial x_{i}}\left(g^{i j} \sqrt{g} \frac{\partial}{\partial x_{j}}\right) .
$$

makes $\Delta_{g}$ is negative, but many authors call $-\Delta_{g}$ the Laplacian to avoid the minus signs. Also, the metric $g$ is often fixed and is dropped from the notation.

A less traditional choice is to denote eigenvalues by $\lambda^{2}$ rather than $\lambda$. It is a common convention in microlocal analysis and so we adopt it here. But we warn that $\lambda$ is often used to denote $\Delta$-eigenvalues as is $[\mathbf{D F}, \mathbf{H}]$.

We sometimes denote eigenfunctions of eigenvalue $-\lambda^{2}$ by $\varphi_{\lambda}$ when we only wish to emphasize the corresponding eigenvalue and do not need $\varphi_{\lambda}$ to be part of an orthonormal basis. For instance, when $\Delta_{g}$ has multiplicities as on the standard sphere or rational torus, there are many possible orthonormal bases. But estimates on $\mathcal{H}^{n-1}\left(Z_{\varphi_{\lambda}}\right)$ do not depend on whether $\varphi_{\lambda}$ is included in the orthonormal basis.

Acknowledgments. Thanks to D. Mangoubi, G. Rivière, C. D. Sogge and B. Shiffman for helpful comments/improvements on the exposition, and to S. Dyatlov for a stimulating discussion of $L^{\infty}$ quantum ergodicity.

\section{Basic estimates of eigenfunctions}

We start by collecting some classical elliptic estimates and their applications to eigenfunctions.

First, the general Sobolev estimate: Let $w \in C_{0}^{\infty}(\Omega)$ where $\Omega \subset \mathbb{R}^{n}$ with $n \geq 3$. Then there exists $C>0$ :

$$
\left(\int_{\Omega}|w|^{\frac{2 n}{n-2}}\right)^{\frac{n-2}{n}} \leq C \int_{\Omega}|\nabla w|^{2} .
$$

Next, we recall the Bernstein gradient estimates:

THEOREM 1.1. [DF3] Local eigenfunctions of a Riemannian manifold satisfy: 
(1) $L^{2}$ Bernstein estimate:

$$
\left(\int_{B(p, r)}\left|\nabla \varphi_{\lambda}\right|^{2} d V\right)^{1 / 2} \leq \frac{C \lambda}{r}\left(\int_{B(p, r)}\left|\varphi_{\lambda}\right|^{2} d V\right)^{1 / 2} .
$$

(2) $L^{\infty}$ Bernstein estimate: There exists $K>0$ so that

$$
\max _{x \in B(p, r)}\left|\nabla \varphi_{\lambda}(x)\right| \leq \frac{C \lambda^{K}}{r} \max _{x \in B(p, r)}\left|\varphi_{\lambda}(x)\right| .
$$

(3) Dong's improved bound:

$$
\begin{aligned}
& \max _{B_{r}(p)}\left|\nabla \varphi_{\lambda}\right| \leq \frac{C_{1} \sqrt{\lambda}}{r} \max _{B_{r}(p)}\left|\varphi_{\lambda}\right| \\
& \text { for } r \leq C_{2} \lambda^{-1 / 4} .
\end{aligned}
$$

Another well-known estimate is the doubling estimate:

Theorem 1.2. (Donnelly-Fefferman, Lin) and $[\mathbf{H}]$ (Lemma 6.1.1) Let $\varphi_{\lambda}$ be a global eigenfunction of a $C^{\infty}(M, g)$ there exists $C=C(M, g)$ and $r_{0}$ such that for $0<r<r_{0}$,

$$
\frac{1}{\operatorname{Vol}\left(B_{2 r}(a)\right)} \int_{B_{2 r}(a)}\left|\varphi_{\lambda}\right|^{2} d V_{g} \leq e^{C \lambda} \frac{1}{\operatorname{Vol}\left(B_{r}(a)\right)} \int_{B_{r}(a)}\left|\varphi_{\lambda}\right|^{2} d V_{g} .
$$

Further,

$$
\max _{B(p, r)}\left|\varphi_{\lambda}(x)\right| \leq\left(\frac{r}{r^{\prime}}\right)^{C \lambda} \max _{x \in B\left(p, r^{\prime}\right)}\left|\varphi_{\lambda}(x)\right|, \quad\left(0<r^{\prime}<r\right) .
$$

The doubling estimates imply the vanishing order estimates. Let $a \in M$ and suppose that $u(a)=0$. By the vanishing order $\nu(u, a)$ of $u$ at $a$ is meant the largest positive integer such that $D^{\alpha} u(a)=0$ for all $|\alpha| \leq \nu$.

TheOREM 1.3. Suppose that $M$ is compact and of dimension $n$. In the case of a global eigenfunction, $\nu\left(\varphi_{\lambda}, a\right) \leq C(M, g) \lambda$.

We now recall quantitative lower bound estimates. They follow from doubling estimates and also from Carleman inequalities.

THEOREM 1.4. Suppose that $M$ is compact and that $\varphi_{\lambda}$ is a global eigenfunction, $\Delta \varphi_{\lambda}=\lambda^{2} \varphi_{\lambda}$. Then for all $p, r$, there exist $C, C^{\prime}>0$ so that

$$
\max _{x \in B(p, r)}\left|\varphi_{\lambda}(x)\right| \geq C^{\prime} e^{-C \lambda} .
$$

Local lower bounds on $\frac{1}{\lambda} \log \left|\varphi_{\lambda}^{\mathbb{C}}\right|$ follow from doubling estimates. They imply that there exists $A, \delta>0$ so that, for any $\zeta_{0} \in \overline{M_{\tau / 2}}$,

$$
\sup _{\zeta \in B_{\delta}\left(\zeta_{0}\right)}\left|\varphi_{\lambda}(\zeta)\right| \geq C e^{-A \lambda}
$$

To see how doubling estimates imply Theorem 1.4, we observe that there exists a point $x_{0} \in M$ so that $\left|\varphi_{\lambda}\left(x_{0}\right)\right| \geq 1$. Any point of $\bar{M}_{\tau / 2}$ can be linked 
to this point by a smooth curve of uniformly bounded length. We then choose $\delta$ sufficiently small so that the $\delta$-tube around the curve lies in $M_{\tau}$ and link $B_{\delta}(\zeta)$ to $B_{\delta}\left(x_{0}\right)$ by a chain of $\delta$-balls in $M_{\tau}$ where the number of links in the chain is uniformly bounded above as $\zeta$ varies in $M_{\tau}$. If the balls are denoted $B_{j}$ we have $\sup _{B_{j+1}}\left|\varphi_{\lambda}\right| \leq e^{\beta \lambda} \sup _{B_{j}}\left|\varphi_{\lambda}\right|$ since $B_{j+1} \subset 2 B_{j}$. The growth estimate implies that for any ball $B, \sup _{2 B}\left|\varphi_{\lambda}\right| \leq e^{C \lambda} \sup _{B}\left|\varphi_{\lambda}\right|$. Since the number of balls is uniformly bounded,

$$
1 \leq \sup _{B_{\delta}\left(x_{0}\right)}\left|\varphi_{\lambda}\right| \leq e^{A \lambda} \sup _{B_{\delta}(\zeta)}\left|\varphi_{\lambda}\right|
$$

proving Theorem 1.4.

As an illustration, Gaussian beams such as highest weight spherical harmonics decay at a rate $e^{-C \lambda d^{2}(x, \gamma)}$ away from a stable elliptic orbit $\gamma$. Hence if the closure of an open set is disjoint from $\gamma$, one has a uniform exponential decay rate which saturate the lower bounds.

We now recall sup-norm estimates of eigenfunctions which follow from the local Weyl law:

$$
\Pi_{\lambda}(x, x):=\sum_{\lambda_{\nu} \leq \lambda}\left|\varphi_{\nu}(x)\right|^{2}=(2 \pi)^{-n} \int_{p(x, \xi) \leqslant \lambda} d \xi+R(\lambda, x)
$$

with uniform remainder bounds

$$
|R(\lambda, x)| \leqslant C \lambda^{n-1}, \quad x \in M .
$$

Since the integral in the local Weyl law is a continuous function of $\lambda$ and since the spectrum of the Laplacian is discrete, this immediately gives

$$
\sum_{\lambda_{\nu}=\lambda}\left|\varphi_{\nu}(x)\right|^{2} \leqslant 2 C \lambda^{n-1}
$$

which in turn yields

$$
\left\|\varphi_{\lambda}\right\|_{C^{0}}=O\left(\lambda^{\frac{n-1}{2}}\right)
$$

on any compact Riemannian manifold.

1.1. $L^{p}$ estimates. The classical Sogge estimates state that, for any compact Riemannian manifold of dimension $n$, we have

$$
\frac{\left\|\varphi_{\lambda}\right\|_{p}}{\left\|\varphi_{\lambda}\right\|_{2}}=O\left(\lambda^{\delta(p)}\right), \quad 2 \leqslant p \leqslant \infty
$$

where

$$
\delta(p)=\left\{\begin{array}{l}
n\left(\frac{1}{2}-\frac{1}{p}\right)-\frac{1}{2}, \quad \frac{2(n+1)}{n-1} \leqslant p \leqslant \infty \\
\frac{n-1}{2}\left(\frac{1}{2}-\frac{1}{p}\right), \quad 2 \leqslant p \leqslant \frac{2(n+1)}{n-1} .
\end{array}\right.
$$

Since we often use surfaces as an illustrantion, we note that in dimension 2 one has for $\lambda \geqslant 1$,

$$
\left\|\varphi_{\lambda}\right\|_{L^{p}(M)} \leqslant C \lambda^{\frac{1}{2}\left(\frac{1}{2}-\frac{1}{p}\right)}\left\|\varphi_{\lambda}\right\|_{L^{2}(M)}, \quad 2 \leqslant p \leqslant 6,
$$

and

$$
\left\|\varphi_{\lambda}\right\|_{L^{p}(M)} \leqslant C \lambda^{2\left(\frac{1}{2}-\frac{1}{p}\right)-\frac{1}{2}}\left\|\varphi_{\lambda}\right\|_{L^{2}(M)}, \quad 6 \leqslant p \leqslant \infty .
$$


These estimates are also sharp for the round sphere $S^{2}$. The first estimate, (13), is saturated by highest weight spherical harmonics. The second estimate, (14), is sharp due to the zonal functions on $S^{2}$, which concentrate at points. We go over these examples in $\S 3.2$.

\section{Volume and equidistribution problems on nodal sets and level sets}

We begin the survey by stating some of the principal problems an results regarding nodal sets and more general level sets. Some of the problems are intentionally stated in vague terms that admit a number of rigorous formulations.

2.1. Hypersurface areas of nodal sets. One of the principal problems on nodal sets is to measure their hypersurface volume. In the real analytic case, Donnelly-Fefferman ( $[\mathbf{D F}]$ (see also $[\mathbf{L i n}]))$ proved:

THeORem 2.1. Let $(M, g)$ be a compact real analytic Riemannian manifold, with or without boundary. Then there exist $c_{1}, C_{2}$ depending only on $(M, g)$ such that

$$
c_{1} \lambda \leq \mathcal{H}^{m-1}\left(Z_{\varphi_{\lambda}}\right) \leq C_{2} \lambda, \quad\left(\Delta \varphi_{\lambda}=\lambda^{2} \varphi_{\lambda} ; c_{1}, C_{2}>0\right) .
$$

The bounds were conjectured by S. T. Yau $[\mathbf{Y 1}, \mathbf{Y 2}]$ for all $C^{\infty}(M, g)$, but this remains an open problem. The lower bound was proved for all $C^{\infty}$ metrics for surfaces, i.e. for $n=2$ by Brüning $[\mathbf{B r}]$. For general $C^{\infty}$ metrics the sharp upper and lower bounds are not known, although there has been some recent progress that we consider below.

The nodal hypersurface bounds are consistent with the heuristic that $\varphi_{\lambda}$ is the analogue on a Riemannian manifold of a polynomial of degree $\lambda$, since the hypersurface volume of a real algebraic variety is bounded by its degree.

2.2. Equidistribution of nodal sets in the real domain. The equidistribution problem for nodal sets is to study the behavior of the integrals (3) of general continuous functions $f$ over the nodal set. Here, we normalize the delta-function on the nodal set by the conjectured surface volume of $\S 2.1$. More precisely:

Problem Find the weak* limits of the family of measures $\left\{\frac{1}{\lambda_{j}} d S_{\lambda_{j}}\right\}$.

Note that in the $C^{\infty}$ case we do not even know if this family has uniformly bounded mass. The high-frequency limit is the semi-classical limit and generally signals increasing complexity in the 'topography' of eigenfunctions.

Heuristics from quantum chaos suggests that eigenfunctions of quantum chaotic systems should behave like random waves. The random wave model is defined and studied in $[\mathbf{Z 4}]$ (see $\S 11$ ), and it is proved (see Theorem 11.1) 
that if one picks a random sequence $\left\{\psi_{\lambda_{j}}\right\}$ of random waves of increasing frequency, then almost surely

$$
\frac{1}{\lambda_{j}} \int_{\mathcal{H}_{\psi_{\lambda_{j}}}} f d S_{\lambda_{j}} \rightarrow \frac{1}{\operatorname{Vol}(M)} \int_{M} f d V_{g},
$$

i.e. their nodal sets become equidistributed with respect to the volume form on $M$. Hence the heuristic principle leads to the conjecture that nodal sets of eigenfunctions of quantum chaotic systems should become equidistributed according to the volume form.

The conjecture for eigenfunctions (rather than random waves) is far beyond any current techniques and serves mainly as inspiration for studies of equidistribution of nodal sets.

A yet more speculative conjecture in quantum chaosis that the nodal sets should tend to $C L E_{6}$ curves in critical percolation. CLE refers to conformal loop ensembles, which are closed curves related to $S L E$ curves. As above, this problem is motivated by a comparision to random waves, but for these the problem is also completely open. In $\S 12$ we review the heuristic principles which started in condensed matter physics [KH, KHS, Isi, IsiK, Wei] before migrating to quantum chaos [BS, BS2, FGS, BGS, SS, EGJS]. It is dubious that such speculative conjectures can be studied rigorously in the forseeable future, but we include them to expose the reader to the questions that are relevant to physicists.

2.3. $L^{1}$ norms and nodal sets. Besides nodal sets it is of much current interest to study $L^{p}$ norms of eigenfunctions globally on $(M, g)$ and also of their restrictions to submanifolds. In fact, recent results show that nodal sets and $L^{p}$ norms are related. For instance, in $\S 4$ we will use the identity

$$
\left\|\varphi_{\lambda}\right\|_{L^{1}}=\frac{1}{\lambda^{2}} \int_{Z_{\varphi_{\lambda}}}\left|\nabla \varphi_{\lambda}\right| d S
$$

relating the $L^{1}$ norm of $\varphi_{\lambda}$ to a weighted integral over $Z_{\varphi_{\lambda}}$ to obtain lower bounds on $\mathcal{H}^{n-1}\left(Z_{\varphi_{\lambda}}\right)$. See $(21)$.

Obtaining lower bounds on $L^{1}$ norms of eigenfunctions is closely related to finding upper bounds on $L^{4}$ norms. The current bounds are not sharp enough to improve nodal set bounds.

2.4. Critical points and values. A closely related problem in the 'topography' of Laplace eigenfunctions $\varphi_{\lambda}$ is to determine the asymptotic distribution of their critical points

$$
C\left(\varphi_{\lambda}\right)=\left\{x: \nabla \varphi_{\lambda}(x)=0\right\}
$$

This problem is analogous to that of measuring the hypersurface area $\mathcal{H}^{n-1}\left(Z_{\lambda}\right)$ of the nodal (zero) set of $\varphi_{\lambda}$, but it is yet more complicated due to the instability of the critical point set as the metric varies. For a generic metric, all eigenfunctions are Morse functions and the critical point set is 
discrete. One may ask to count the number of critical points asymptotically as $\lambda \rightarrow \infty$. But there exist metrics (such as the flat metric on the torus, or the round metric on the sphere) for which the eigenfunctions have critical hypersurfaces rather than points. To get around this obstruction, we change the problem from counting critical points to counting critical values

$$
C V\left(\varphi_{\lambda}\right)=\left\{\varphi_{\lambda}(x): \nabla \varphi_{\lambda}(x)=0\right\} .
$$

Since a real analytic function on a compact real analytic manifold has only finitely many critical values, eigenfunctions of real analytic Riemannian manifolds $(M, g)$ have only finitely many critical values and we can ask to count them. See Conjecture 6.2 for an apparently plausible bound. Moreover for generic real analytic metrics, all eigenfunctions are Morse functions and there exists precisely one critical point for each critical value. Thus, in the generic situation, counting critical values is equivalent to counting critical points. To our knowledge, there are no results on this problem, although it is possible to bound the $\mathcal{H}^{n-1}$-measure of $C\left(\varphi_{\lambda}\right)$ (see Theorem [Ba]). However $\mathcal{H}^{n-1}\left(C\left(\varphi_{\lambda}\right)\right)=0$ in the generic case and in special cases where it is not zero the method is almost identical to bounds on the nodal set. Thus, such results bypass all of the difficulties in counting critical values. We will present one new (unpublished) result which generalizes (16) to critical points. But the resulting identity is much more complicated than for zeros.

Singular points are critical points which occur on the nodal sets. We recall (see $[\mathbf{H}, \mathbf{H H L}, \mathbf{H H O N}]$ ) that the the singular set

$$
\Sigma\left(\varphi_{\lambda}\right)=\left\{x \in Z_{\varphi_{\lambda}}: \nabla \varphi_{\lambda}(x)=0\right\}
$$

satisfies $\mathcal{H}^{n-2}\left(\Sigma\left(\varphi_{\lambda}\right)\right)<\infty$. Thus, outside of a codimension one subset, $Z_{\varphi_{\lambda}}$ is a smooth manifold, and the Riemannian surface measure $d S=\iota \frac{\nabla \varphi_{\lambda}}{\left|\nabla \varphi_{\lambda}\right|} d V_{g}$ on $Z_{\varphi_{\lambda}}$ is well-defined. We refer to [HHON, H, HHL, HS] for background.

2.5. Inradius. It is known that in dimension two, the minimal possible area of a nodal domain of a Euclidean eigenfunction is $\pi\left(\frac{j_{1}}{\lambda}\right)^{2}$. This follows from the two-dimensional Faber-Krahn inequality,

$$
\lambda_{k}(\Omega) \operatorname{Area}(D)=\lambda_{1}(D) \operatorname{Area}(D) \geq \pi j_{1}^{2}
$$

where $D$ is a nodal domain in $\Omega$. In higher dimensions, the Faber-Krahn inequality shows that on any Riemannian manifold the volume of any nodal domain is $\geq C \lambda^{-n}[\mathbf{E K}]$.

Another size measure of a nodal domain is its inradius $r_{\lambda}$, i.e. the radius of the largest ball contained inside the nodal domain. As can be seen from computer graphics (see e.g. [HEJ]), there are a variety of 'types' of nodal components. In [Man3], Mangoubi proves that

$$
\frac{C_{1}}{\lambda} \geq r_{\lambda} \geq \frac{C_{2}}{\lambda^{\frac{1}{2} k(n)}(\log \lambda)^{2 n-4}},
$$

where $k(n)=n^{2}-15 n / 8+1 / 4$; note that eigenvalues in [Man] are denoted $\lambda$ while here we denote them by $\lambda^{2}$. In dimension 2 , it is known (loc.cit.) 
that

$$
\frac{C_{1}}{\lambda} \geq r_{\lambda} \geq \frac{C_{2}}{\lambda}
$$

2.6. Decompositions of $M$ with respect to $\varphi_{\lambda}$. There are two natural decompositions (partitions) of $M$ associated to an eigenfunction (or any smooth function).

(i) Nodal domain decomposition.

First is the decomposition of $M$ into nodal domains of $\varphi_{\lambda}$. As in [PS] we denote the collection of nodal domains by $\mathcal{A}\left(\varphi_{\lambda}\right)$ and denote a nodal domain by $A$. Thus,

$$
M \backslash Z_{\varphi_{\lambda}}=\bigcup_{A \in \mathcal{A}\left(\varphi_{\lambda}\right)} A .
$$

When 0 is a regular value of $\varphi_{\lambda}$ the level sets are smooth hypersurfaces and one can ask how many components of $Z_{\varphi_{\lambda}}$ occur, how many components of the complement, the topological types of components or the combinatorics of the set of domains. When 0 is a singular value, the nodal set is a singular hypersurface and can be connected but one may ask similar questions taking multiplicities of the singular points into account.

To be precise, let

$$
\mu\left(\varphi_{\lambda}\right)=\# \mathcal{A}\left(\varphi_{\lambda}\right), \quad \nu\left(\varphi_{\lambda}\right)=\# \text { components of } Z\left(\varphi_{\lambda}\right) .
$$

The best-known problem is to estimate $\mu\left(\varphi_{\lambda}\right)$. According to the Courant nodal domain theorem, $\mu\left(\varphi_{\lambda_{n}}\right) \leq n$. In the case of spherical harmonics, where many orthonormal bases are possible, it is better to estimate the number in terms of the eigenvalue, and the estimate has the form $\mu\left(\varphi_{\lambda}\right) \leq$ $C(g) \lambda^{m}$ where $m=\operatorname{dim} M$ and $C(g)>0$ is a constant depending on $g$. In dimension 2, Pleijel used the Faber-Krahn theorem to improve the bound to

$$
\limsup _{\lambda \rightarrow \infty} \frac{\mu\left(\varphi_{\lambda}\right)}{\lambda^{2}} \leq \frac{4}{j_{0}^{2}}<0.69
$$

where $j_{0}$ is the smallest zero of the $J_{0}$ Bessel function.

A wide variety of behavior is exhibited by spherical harmonics of degree $N$. We review the definitions below. The even degree harmonics are equivalent to real projective plane curves of degree $N$. But each point of $\mathbb{R P}^{2}$ corresponds to a pair of points of $S^{2}$ and at most one component of the nodal set is invariant under the anti-podal map. For other components, the anti-podal map takes a component to a disjoint component. Thus there are essentially twice the number of components in the nodal set as components of the associated plane curve.

As discussed in [Ley], one has:

- Harnack's inequality: the number of components of any irreducible real projective plane curve is bounded by $g+1$ where $g$ is the genus of the curve. 
- If $p$ is a real projective plane curve of degree $N$ then its genus is given by Noether's formula

$$
g=\frac{(N-1)(N-2)}{2}-\sum_{\text {singular points } \mathrm{x}} \frac{\operatorname{ord}_{p}(x)\left(\operatorname{ord}_{p}(x)-1\right)}{2}
$$

where $\operatorname{ord}_{p}(x)$ is the order of vanishing of $\varphi_{\lambda}$ at $x$. Thus, the number of components is $\leq \frac{(N-1)(N-2)}{2}+1$ for a non-singular irreducible plane curve of degree $N$.

Curves which achieve the maximum are called $M$-curves. Also famous are Harnack curves, which are $M$ curves for which there exist three distinct lines $\ell_{j}$ of $\mathbb{R P}^{2}$ and three distinct arcs $a_{j}$ of the curve on one component so that $\# a_{j} \cap \ell_{j}=N$. It follows from Pleijel's bound that nodal sets of spherical harmonics cannot be maximal for large $N$, since half of the Pleijel bound is roughly $.35 N^{2}$ which is below the threshold $.5 N^{2}+O(N)$ for maximal curves.

Associated to the collection of nodal domains is its incidence graph $\Gamma_{\lambda}$, which has one vertex for each nodal domain, and one edge linking each pair of nodal domains with a common boundary component. Here we assume that 0 is a regular value of $\varphi_{\lambda}$ so that the nodal set is a union of embedded submanifolds. The Euler characteristic of the graph is the difference beween the number of nodal domains and nodal components. In the non-singular case, one can convert the nodal decomposition into a cell decomposition by attaching a one cell between two adjacent components, and then one has $\mu\left(\varphi_{\lambda}\right)=\nu\left(\varphi_{\lambda}\right)+1$ (see Lemma 8 of $[$ Ley $]$ ).

The possible topological types of arrangements of nodal components of spherical harmonics is studied in $[\mathbf{E J N}]$. They prove that for any $m \leq N$ with $N-m$ even and for every set of $m$ disjoint closed curves whose union is invariant with respect to the antipodal map, there exists an eigenfunction whose nodal set has the topological type of the union of curves. Note that these spherical harmonics have relatively few nodal domains compared to the Pleijel bound. It is proved in [NS] that random spherical harmonics have $a N^{2}$ nodal components for some (undetermined) $a>0$.

\section{Morse-Smale decomposition}

For generic metrics, all eigenfunctions are Morse functions [U]. Suppose that $f: M \rightarrow \mathbb{R}$ is a Morse function. For each critical point $p$ let $W^{s} p$ (the descending cell through $\mathrm{p}$ ) denote the union of the downward gradient flow lines which have $p$ as their initial point, i.e. their $\alpha$-limit point. Then $W_{p}$ is a cell of dimension $\lambda_{p}=$ number of negative eigenvalues of $H_{p} f$. By the Morse-Smale decomposition we mean the decomposition

$$
M=\bigcup_{p: d f(p)=0} W_{p}^{s}
$$

It is not a good cell decomposition in general. If we change $f$ to $-f$ we get the decomposition into ascending cells $M=\bigcup_{p: d f(p)=0} W_{p}^{u}$. If the intersections 


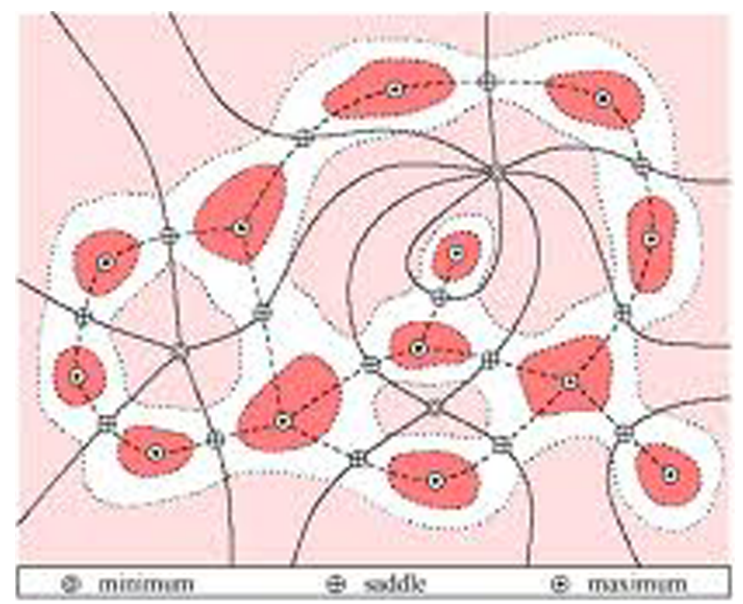

Figure 1. A Morse complex with solid stable 1-manifolds and dashed unstable 1-manifolds. In drawing the dotted isolines we assume that all saddles have height between all minima and all maxima.

$W_{p}^{s} \cap W_{q} u$ are always transversal then $\nabla f$ is said to be transversal. In this case $\operatorname{dim}\left(W_{p}^{s} \cap W_{q}^{u}\right)=\lambda_{p}-\lambda_{q}+1$ and the number of gradient curves joining two critical points whose Morse index differs by 1 is finite.

We are mainly interested in the stable cells of maximum dimension, i.e. basins of attraction of the gradient flow to each local minimum. We then have the partition

$$
M=\bigcup_{p \text { a local min }} W_{p}^{u}
$$

This decomposition is somtimes used in condensed matter physics (see e.g. [Wei]) and in computational shape analysis [Reu]. In dimension two, the surface is partitioned into 'polygons' defined by the basins of attraction of the local minima of $\varphi$. The boundaries of these polygons are gradient lines of $\varphi$ which emanate from saddle points. The vertices occur at local maxima.

An eigenfunction is a Neumann eigenfunction in each basin since the boundary is formed by integral curves of $\nabla \varphi_{\lambda}$. Possibly it is 'often' the first non-constant Neumann eigenfunction (analogously to $\varphi_{\lambda}$ being the lowest Dirichlet eigenfunction in each nodal domain), but this does not seem obvious. Hence it is not clear how to relate the global eigenvalue $\lambda^{2}$ to the Neumann eigenvalues of the basins, which would be useful in understanding the areas or diameters of these domains. Note that

$$
\int_{W_{p}^{u}} \varphi_{j} d V=\int_{\partial W_{p}^{u}} \nabla \varphi_{\lambda} \cdot \nu d S=0
$$

where $\nu$ is the unit normal to $\partial W_{p}^{u}$, since $\nabla \varphi_{\lambda}$ is tangent to the boundary. In particular, the intersection $Z_{\varphi_{\lambda}} \cap W_{p}^{u}$ is non-empty and is a connected 


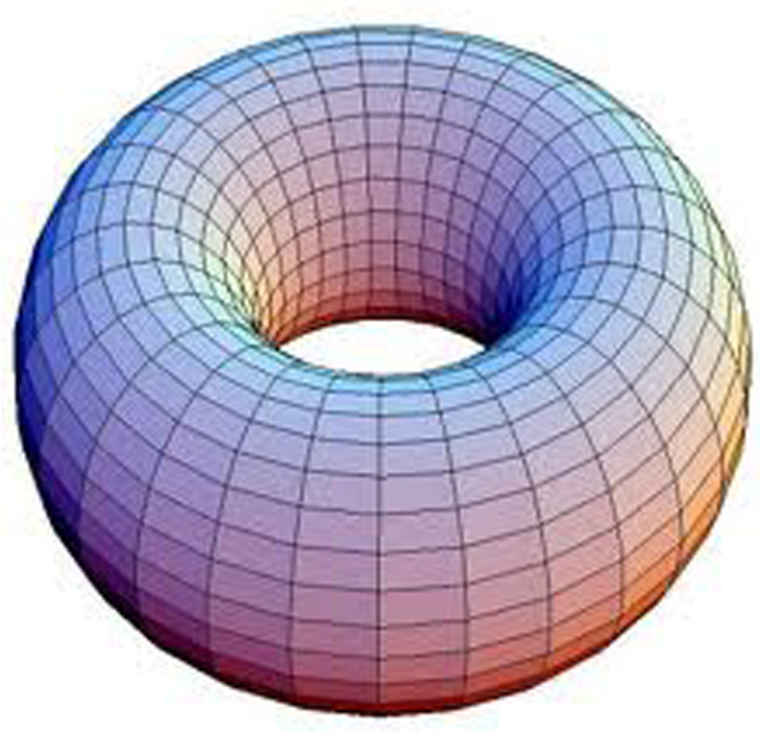

FigURE 2

hypersurface which separates $W_{p}^{s}$ into two components on which $\varphi_{\lambda}$ has a fixed sign. To our knowledge, there do not exist rigorous results bounding the number of local minima from above or below, i.e. there is no analogue of the Courant upper bound for the number of local minima basins. It is possible to obtain statstical results on the asymptotic expected number of local minima, say for random spherical harmonics of degree $N$. The methods of $[\mathbf{D S Z}]$ adapt to this problem if one replaces holomorphic Szegö kernels by spectral projections (see also [Nic].) Thus, in a statistical sense it is much simpler to count the number of "Neumann domains" or Morse-Smale basins than to count nodal domains as in [NS].

\section{Examples}

Before proceeding to rigorous results, we go over a number of explicitly solvable examples. Almost by definition, they are highly non-generic and in fact represent the eigenfunctions of quantum integrable systems. Aside from being explicitly solvable, the eigenfunctions of this section are extremals for a number of problems.

3.1. Flat tori. The basic real valued eigenfunctions are $\varphi_{k}(x)=$ $\sin \langle k, x\rangle$ or $\cos \langle k, x\rangle \quad\left(k \in \mathbb{Z}^{n}\right)$ on the flat torus $\mathbf{T}=\mathbb{R}^{n} / \mathbb{Z}^{n}$. The zero set consists of the hyperplanes $\langle k, x\rangle=0 \bmod 2 \pi$ or in other words $\left\langle x, \frac{k}{|k|}\right\rangle \in \frac{1}{2 \pi|k|} \mathbb{Z}$. Thus the normalized delta function $\left.\frac{1}{|k|} d S\right|_{Z_{\varphi_{k}}}$ tends to uniform distribution along rays in the lattice $\mathbb{Z}^{n}$. The lattice arises as the joint spectrum of the commuting operators $D_{j}=\frac{\partial}{i \partial x_{j}}$ and is a feature of quantum integrable systems. 
The critical point equation for $\cos \langle k, x\rangle$ is $k \sin \langle k, x\rangle=0$ and is thus the same as the nodal equation. In particular, the critical point sets are hypersurfaces in this case. There is just one critical value $=1$.

Instead of the square torus we could consider $\mathbb{R}^{n} / L$ where $L \subset \mathbb{R}^{n}$ is a lattice of full rank. Then the joint spectrum becomes the dual lattice $L^{*}$ and the eigenfunctions are $\cos \langle k, x\rangle, \sin \langle k, x\rangle$ with $k \in L^{*}$.

The real eigenspace $\mathcal{H}_{\lambda}=\mathbb{R}-\operatorname{span}\{\sin \langle k, x\rangle, \cos \langle k, x\rangle:|k|=\lambda\}$ is of multiplicity 2 for generic $L$ but has unbounded multiplicity in the case of $L=\mathbb{Z}^{n}$ and other rational lattices. In that case, one may take linear combinations of the basic eigenfunctions and study their nodal and critcal point sets. For background, some recent results and further references we refer to $[\mathbf{B Z}]$.

3.2. Spherical harmonics on $S^{2}$. The spectral decomposition for the Laplacian is the orthogonal sum of the spaces of spherical harmonics of degree $N$,

$$
L^{2}\left(S^{2}\right)=\bigoplus_{N=0}^{\infty} V_{N},\left.\quad \Delta\right|_{V_{N}}=\lambda_{N} I d .
$$

The eigenvalues are given by $\lambda_{N}^{S^{2}}=N(N+1)$ and the multiplicities are given by $m_{N}=2 N+1$. A standard basis is given by the (complex valued) spherical harmonics $Y_{m}^{N}$ which transform by $e^{i m \theta}$ under rotations preserving the poles.

The $Y_{m}^{N}$ are complex valued, so we study the nodal sets of their real and imaginary parts. They are separable, i.e. factor as $C_{N, m} P_{m}^{N}(r) \sin (m \theta)$ (resp. $\cos (m \theta)$ where $P_{m}^{N}$ is an associated Legendre function. Thus the nodal sets of these special eigenfunctions form a checkerboard pattern that can be explicitly determined from the known behavior of zeros of associated Legendre functions. See the first image in the illustration below.

Among the basic spherical harmonics, there are two special ones: the zonal spherical harmonics (i.e. the rotationally invariant harmonics) and the highest weight spherical harmonics. Their nodal sets and intensity plots are graphed in the bottom two images, respectively.

Since the zonal spherical harmonics $Y_{0}^{N}$ on $S^{2}$ are real-valued and rotationally invariant, their zero sets consist of a union of circles, i.e. orbits of the $S^{1}$ rotation action around the third axis. It is well known that $Y_{0}^{N}(r)=\sqrt{\frac{(2 N+1)}{2 \pi}} P_{N}(\cos r)$, where $P_{N}$ is the $N$ th Legendre function and the normalizing constant is chosen so that $\left\|Y_{0}^{N}\right\|_{L^{2}\left(S^{2}\right)}=1$, i.e. $4 \pi \int_{0}^{\pi / 2}\left|P_{N}(\cos r)\right|^{2} d v(r)=1$, where $d v(r)=\sin r d r$ is the polar part of the area form. Thus the circles occur at values of $r$ so that $P_{N}(\cos r)=0$. All zeros of $P_{N}(x)$ are real and it has $N$ zeros in $[-1,1]$. It is classical that the zeros $r_{1}, \ldots, r_{N}$ of $P_{N}(\cos r)$ in $(0, \pi)$ become uniformly distributed with 

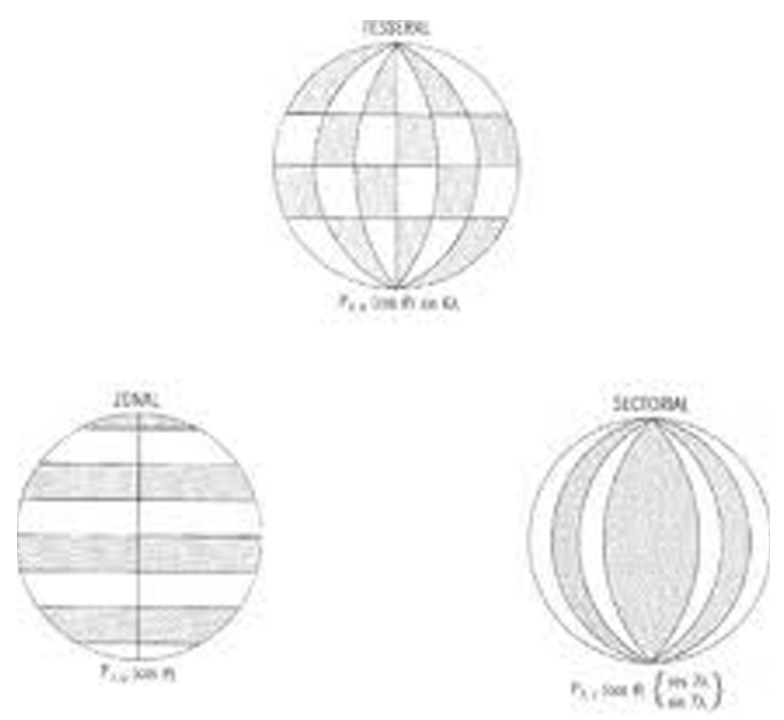

Figure 3. Examples of the different kinds of spherical harmonics.

respect to $d r[\mathbf{S z}]$. It is also known that $P_{N}$ has $N-1$ distinct critical points $[\mathbf{C}, \mathbf{S z 2}]$ and so the critical points of $Y_{0}^{N}$ is a union of $N-1$ lattitude circles.

We now consider real or imaginary parts of highest weight spherical harmonics $Y_{N}^{N}$. Up to a scalar multiple, $Y_{N}\left(x_{1}, x_{2}, x_{2}\right)=\left(x_{1}+i x_{2}\right)^{N}$ as a harmonic polynomial on $\mathbb{R}^{3}$. It is an example of a Gaussian beams along a closed geodesic $\gamma$ (such as exist on equators of convex surfaces of revolution). See $[\mathbf{R}]$ for background on Gaussian beams on Riemannian manifolds.

The real and imaginary parts are of the form $P_{N}^{N}(\cos r) \cos N \theta$, $P_{N}^{N}(\cos r) \sin N \theta$ where $P_{N}^{N}(x)$ is a constant multiple of $\left(1-x^{2}\right)^{N / 2}$ so $P_{N}^{N}(\cos r)=(\sin r)^{N}$. The factors $\sin N \theta, \cos N \theta$ have $N$ zeros on $(0,2 \pi)$. The Legendre funtions satisfy the recursion relation $P_{\ell+1}^{\ell+1}=$ $-(2 \ell+1) \sqrt{1-x^{2}} P_{\ell}^{\ell}(x)$ with $P_{0}^{0}=1$ and therefore have no real zeros away from the poles. Thus, the nodal set consists of $N$ circles of longitude with equally spaced intersections with the equator.

The critical points are solutions of the pair of equations $\frac{d}{d r} P_{N}^{N}(r) \cos N \theta$ $=0, P_{N}^{N} \sin N \theta=0$. Since $P_{N}^{N}$ has no zeros away from the poles, the second equation forces the zeros to occur at zeros of $\sin N \theta$. But then $\cos N \theta \neq 0$ so the zeros must occur at the zeros of $\frac{d}{d r} P_{N}^{N}(r)$. The critical points only occur when $\sin r=0$ or $\cos r=0$ on $(0, \pi)$. There are critical points at the poles where $Y_{N}^{N}$ vanishes to order $N$ and there is a local maximum at the value $r=\frac{\pi}{2}$ of the equator. Thus, $\operatorname{Re} Y_{N}^{N}$ has $N$ isolated critical points on the equator and multiple critical points at the poles.

We note that $\left|\operatorname{Re} Y_{N}^{N}\right|^{2}$ is a Gaussian bump with peak along the equator in the radial direction. Its radial Gaussian decay implies that it extremely small outside a $N^{\frac{1}{2}}$ tube around the equator. The complement of this tube 
is known in physics as the classically forbidden region. We see that the nodal set stretches a long distance into the classically forbidden region. This creates problems for nodal estimates since exponentially small values (in terms of the eigenvalue) are hard to distinguish from zeros. On the other hand, it has only two (highly multiple) critical points away from the equator.

\subsection{Random spherical harmonics and chaotic eigenfunctions.} The examples above exhibit quite disparate behavior but all are eigenfunctions of quantum integrable systems. We do not review the general results in this case but plan to treat this case in an article in preparation $[\mathbf{Z 9}]$.

Figure 4 contrasts the nodal set behavior with that of random spherical harmonics (left) and a chaotic billiard domain (the graphics are due to E. J. Heller).

\section{Lower bounds on hypersurface areas of nodal sets and level sets in the $C^{\infty}$ case}

In this section we review the lower bounds on $\mathcal{H}^{n-1}\left(Z_{\varphi_{\lambda}}\right)$ from $[\mathbf{C M}$, SoZ, SoZa, HS, HW]. Here

$$
\mathcal{H}^{n-1}\left(Z_{\varphi_{\lambda}}\right)=\int_{Z_{\varphi_{\lambda}}} d S
$$

is the Riemannian surface measure, where $d S$ denotes the Riemannian volume element on the nodal set, i.e. the insert $i o t a_{n} d V_{g}$ of the unit normal into the volume form of $(M, g)$. The main result is:

Theorem 4.1. Let $(M, g)$ be a $C^{\infty}$ Riemannian manifold. Then there exists a constant $C$ independent of $\lambda$ such that

$$
C \lambda^{1-\frac{n-1}{2}} \leq \mathcal{H}^{n-1}\left(Z_{\varphi_{\lambda}}\right) .
$$

We sketch the proof of Theorem 4.1 from $[\mathbf{S o Z}, \mathbf{S o Z}$ a]. The starting point is an identity from [SoZ] (inspired by an identity in [Dong]):

Proposition 4.2. For any $f \in C^{2}(M)$,

$$
\int_{M}\left|\varphi_{\lambda}\right|\left(\Delta_{g}+\lambda^{2}\right) f d V_{g}=2 \int_{Z_{\varphi_{\lambda}}}\left|\nabla_{g} \varphi_{\lambda}\right| f d S,
$$

This identity can be used to obtain some rudimentary but non-trivial information on the limit distribution of nodal sets in the $C^{\infty}$ case; see $§ 4.9$. For the moment we only use it to study hypersurface measures of nodal sets. When $f \equiv 1$ we obtain

\section{Corollary 4.3}

$$
\lambda^{2} \int_{M}\left|\varphi_{\lambda}\right| d V_{g}=2 \int_{Z_{\varphi_{\lambda}}}\left|\nabla_{g} \varphi_{\lambda}\right| f d S,
$$

The lower bound of Theorem 4.1 follows from the identity in Corollary 4.3 and the following lemma: 

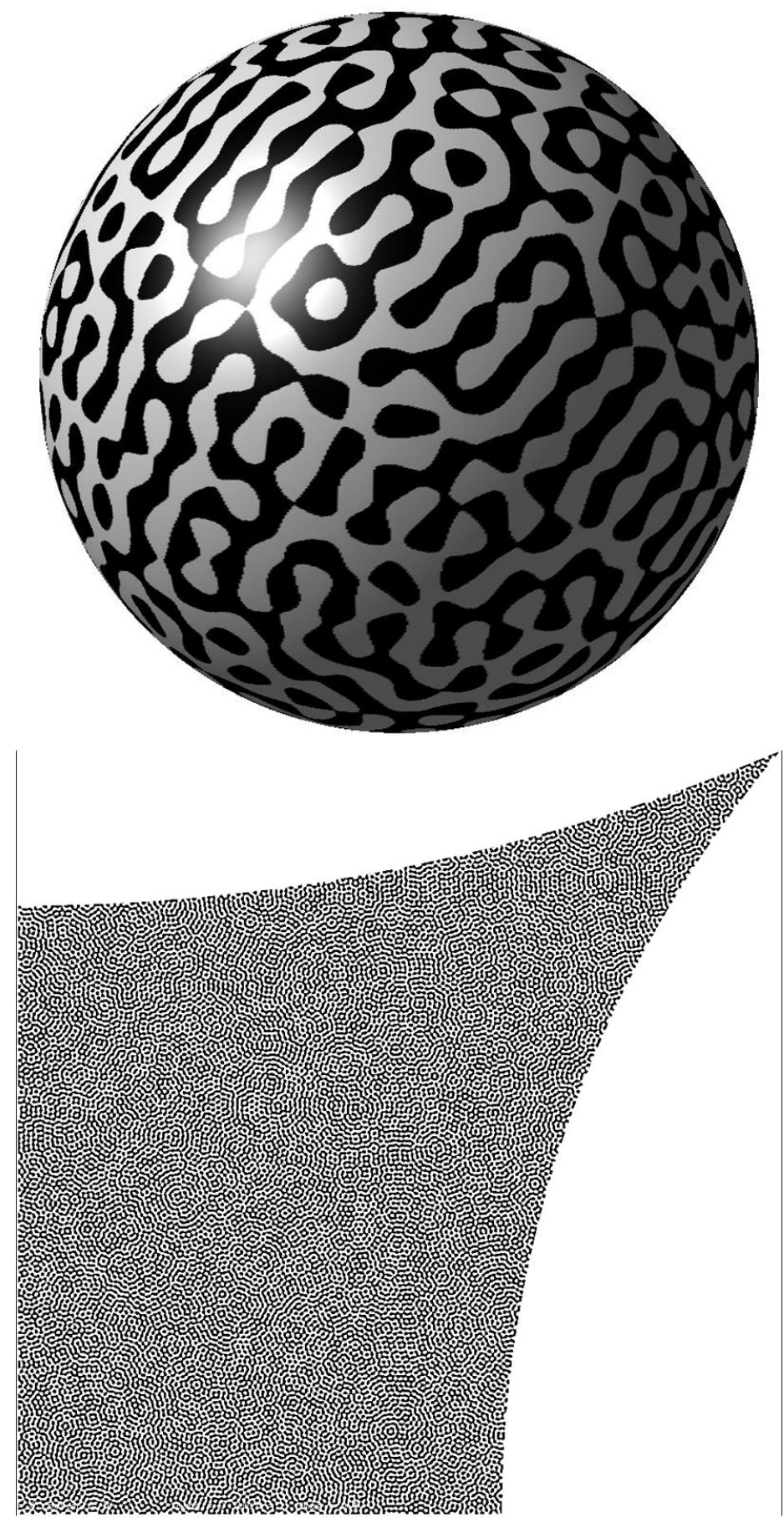

Figure 4 
LEMmA 4.4. If $\lambda>0$ then

$$
\left\|\nabla_{g} \varphi_{\lambda}\right\|_{L^{\infty}(M)} \lesssim \lambda^{1+\frac{n-1}{2}}\left\|\varphi_{\lambda}\right\|_{L^{1}(M)}
$$

Here, $A(\lambda) \lesssim B(\lambda)$ means that there exists a constant independent of $\lambda$ so that $A(\lambda) \leq C B(\lambda)$.

By Lemma 4.4 and Corollary 4.3, we have

$$
\begin{aligned}
\lambda^{2} \int_{M}\left|\varphi_{\lambda}\right| d V=2 \int_{Z_{\lambda}}\left|\nabla_{g} \varphi_{\lambda}\right|_{g} d S & \leqslant 2 \mathcal{H}^{n-1}\left(Z_{\lambda}\right)\left\|\nabla_{g} \varphi_{\lambda}\right\|_{L^{\infty}(M)} \\
& \lesssim 2 \mathcal{H}^{n-1}\left(Z_{\lambda}\right) \lambda^{1+\frac{n-1}{2}}\left\|\varphi_{\lambda}\right\|_{L^{1}(M)} .
\end{aligned}
$$

Thus Theorem 4.1 follows from the somewhat curious cancellation of $\left\|\varphi_{\lambda}\right\|_{L^{1}}$ from the two sides of the inequality.

4.1. Proof of Proposition 4.2. We begin by recalling the co-area formula: Let $f: M \rightarrow \mathbb{R}$ be Lipschitz. Then for any continuous function $u$ on $M$,

$$
\int_{M} u(x) d V=\int_{\mathbb{R}}\left(\int_{f^{-1}(y)} u \frac{d V}{d f}\right) d y
$$

Equivalently,

$$
\int_{M} u(x)\|\nabla f\| d V=\int_{\mathbb{R}}\left(\int_{f^{-1}(y)} u d \mathcal{H}^{n-1}\right) d y .
$$

We refer to $\frac{d V}{d f}$ as the "Leray form" on the level set $\{f=y\}$. Unlike the Riemannian surface measure $d S=d \mathcal{H}^{n-1}$ it depends on the choice of defining function $f$. The surface measures are related by $d \mathcal{H}^{n-1}=|\nabla f| \frac{d V}{d f}$. For background, see Theorem 1.1 of $[\mathbf{H L}]$.

There are several ways to prove the identity of Lemma 4.2. One way to see it is that $d \mu_{\lambda}:=\left(\Delta+\lambda^{2}\right)\left|\varphi_{\lambda}\right| d V=0$ away from $\left\{\varphi_{\lambda}=0\right\}$. Hence this distribution is a positive measure supported on $Z_{\varphi_{\lambda}}$. To determine the coefficient of the surface measure $d S$ we calculate the limit as $\delta \rightarrow 0$ of the integral

$$
\int_{M} f\left(\Delta+\lambda^{2}\right)\left|\varphi_{\lambda}\right| d V=\int_{\left|\varphi_{\lambda}\right| \leq \delta} f\left(\Delta+\lambda^{2}\right)\left|\varphi_{\lambda}\right| d V .
$$

Here $f \in C^{2}(M)$ and with no loss of generality we may assume that $\delta$ is a regular value of $\varphi_{\lambda}$ (by Sard's theorem). By the Gauss-Green theorem,

$$
\begin{gathered}
\int_{\left|\varphi_{\lambda}\right| \leq \delta} f\left(\Delta+\lambda^{2}\right)\left|\varphi_{\lambda}\right| d V-\int_{\left|\varphi_{\lambda}\right| \leq \delta}\left|\varphi_{\lambda}\right|\left(\Delta+\lambda^{2}\right) f d V \\
=\int_{\left|\varphi_{\lambda}\right|=\delta}\left(f \partial_{\nu}\left|\varphi_{\lambda}\right|-\left|\varphi_{\lambda}\right| \partial_{\nu} f\right) d S .
\end{gathered}
$$


Here, $\nu$ is the outer unit normal and $\partial_{\nu}$ is the associated directional derivative. For $\delta>0$, we have

$$
\nu=\frac{\nabla \varphi_{\lambda}}{\left|\nabla \varphi_{\lambda}\right|} \text { on }\left\{\varphi_{\lambda}=\delta\right\}, \quad \nu=-\frac{\nabla \varphi_{\lambda}}{\left|\nabla \varphi_{\lambda}\right|} \text { on }\left\{\varphi_{\lambda}=-\delta\right\}
$$

Letting $\delta \rightarrow 0$ (through the sequence of regular values) we get

$$
\int_{M} f\left(\Delta+\lambda^{2}\right)\left|\varphi_{\lambda}\right| d V=\lim _{\delta \rightarrow 0} \int_{\left|\varphi_{\lambda}\right| \leq \delta} f\left(\Delta+\lambda^{2}\right)\left|\varphi_{\lambda}\right| d V=\lim _{\delta \rightarrow 0} \int_{\left|\varphi_{\lambda}\right|=\delta} f \partial_{\nu}\left|\varphi_{\lambda}\right| d S .
$$

Since $\left|\varphi_{\lambda}\right|= \pm \varphi_{\lambda}$ on $\left\{\varphi_{\lambda}= \pm \delta\right\}$ and by (25), we see that

$$
\begin{aligned}
\int_{M} f\left(\Delta+\lambda^{2}\right)\left|\varphi_{\lambda}\right| d V & =\lim _{\delta \rightarrow 0} \int_{\left|\varphi_{\lambda}\right|=\delta} f \frac{\nabla\left|\varphi_{\lambda}\right|}{|\nabla| \varphi_{\lambda}||} \cdot \nabla\left|\varphi_{\lambda}\right| d S \\
& =\lim _{\delta \rightarrow 0} \sum_{ \pm} \int_{\varphi_{\lambda}= \pm \delta} f\left|\nabla \varphi_{\lambda}\right| d S \\
& =2 \int_{Z_{\varphi_{\lambda}}} f\left|\nabla \varphi_{\lambda}\right| d S
\end{aligned}
$$

The Gauss-Green formula and limit are justified by the fact that the singular set $\Sigma_{\varphi_{\lambda}}$ has codimension two. We refer to $[\mathbf{S o Z}]$ for further details.

\subsection{Proof of Lemma 4.4.}

Proof. The main idea is to construct a designer reproducing kernel for $\varphi_{\lambda}$ of the form

$$
\hat{\rho}\left(\lambda-\sqrt{-\Delta_{g}}\right) f=\int_{-\infty}^{\infty} \rho(t) e^{-i t \lambda} e^{i t \sqrt{-\Delta_{g}}} f d t,
$$

with $\rho \in C_{0}^{\infty}(\mathbb{R})$. It has the spectral expansion,

$$
\chi_{\lambda} f=\sum_{j=0}^{\infty} \hat{\rho}\left(\lambda-\lambda_{j}\right) E_{j} f,
$$

where $E_{j} f$ is the projection of $f$ onto the $\lambda_{j}$ - eigenspace of $\sqrt{-\Delta_{g}}$. Then (26) reproduces $\varphi_{\lambda}$ if $\hat{\rho}(0)=1$. We denote the kernel of $\chi_{\lambda}$ by $K_{\lambda}(x, y)$, i.e.

$$
\chi_{\lambda} f(x)=\int_{M} K_{\lambda}(x, y) f(y) d V(y), \quad(f \in C(M)) .
$$

Assuming $\hat{\rho}(0)=1$, then

$$
\int_{M} K_{\lambda}(x, y) \varphi_{\lambda}(y) d V(y)=\varphi_{\lambda}(x)
$$

To obtain Lemma 4.4, we choose $\rho$ so that the reproducing kernel $K_{\lambda}(x, y)$ is uniformly bounded by $\lambda^{\frac{n-1}{2}}$ on the diagonal as $\lambda \rightarrow+\infty$. It suffices to choose $\rho$ so that $\rho(t)=0$ for $|t| \notin[\varepsilon / 2, \varepsilon]$, with $\varepsilon>0$ less than the injectivity radius of $(M, g)$, then it is proved in Lemma 5.1.3 of [Sog3] that

$$
K_{\lambda}(x, y)=\lambda^{\frac{n-1}{2}} a_{\lambda}(x, y) e^{i \lambda r(x, y)}
$$


where $a_{\lambda}(x, y)$ is bounded with bounded derivatives in $(x, y)$ and where $r(x, y)$ is the Riemannian distance between points. This WKB formula for $K_{\lambda}(x, y)$ is known as a parametrix.

It follows from (28) that

$$
\left|\nabla_{g} K_{\lambda}(x, y)\right| \leqslant C \lambda^{1+\frac{n-1}{2}}
$$

and therefore,

$$
\begin{aligned}
\sup _{x \in M}\left|\nabla_{g} \chi_{\lambda} f(x)\right| & =\sup _{x}\left|\int f(y) \nabla_{g} K_{\lambda}(x, y) d V\right| \\
& \leqslant\left\|\nabla_{g} K_{\lambda}(x, y)\right\|_{L^{\infty}(M \times M)}\|f\|_{L^{1}} \\
& \leqslant C \lambda^{1+\frac{n-1}{2}}\|f\|_{L^{1}} .
\end{aligned}
$$

To complete the proof of Lemma 4.4, we set $f=\varphi_{\lambda}$ and use that $\chi_{\lambda} \varphi_{\lambda}=$ $\varphi_{\lambda}$.

We view $K_{\lambda}(x, y)$ as a designer reproducing kernel, because it is much smaller on the diagonal than kernels of the spectral projection operators $E_{[\lambda, \lambda+1]}=\sum_{j: \lambda_{j} \in[\lambda, \lambda+1]} E_{j}$. The restriction on the support of $\rho$ removes the big singularity on the diagonal at $t=0$. As discussed in [SoZa], it is possible to use this kernel because we only need it to reproduce one eigenfunction and not a whole spectral interval of eigenfunctions.

4.3. Modifications. After an initial modification in $[\mathbf{H W}]$, an interesting application of Proposition 4.2 was used in [HS] to prove

TheOREM 4.5. [HS] For any $C^{\infty}$ compact Riemannian manifold, the $L^{2}$-normalized eigenfunctions satisfy

$$
\mathcal{H}^{n-1}\left(Z_{\varphi_{\lambda}}\right) \geq C \lambda\left\|\varphi_{\lambda}\right\|_{L^{1}}^{2} .
$$

They first apply the Schwarz inequality to get

$$
\lambda^{2} \int_{M}\left|\varphi_{\lambda}\right| d V_{g} \leqslant 2\left(\mathcal{H}^{n-1}\left(Z_{\varphi_{\lambda}}\right)\right)^{1 / 2}\left(\int_{Z_{\varphi_{\lambda}}}\left|\nabla_{g} \varphi_{\lambda}\right|^{2} d S\right)^{1 / 2} .
$$

They then use the test function

$$
f=\left(1+\lambda^{2} \varphi_{\lambda}^{2}+\left|\nabla_{g} \varphi_{\lambda}\right|_{g}^{2}\right)^{\frac{1}{2}}
$$

in Proposition 4.2 to show that

$$
\int_{Z_{\varphi_{\lambda}}}\left|\nabla_{g} \varphi_{\lambda}\right|^{2} d S \leq \lambda^{3}
$$

A simpler approach to the last step was suggested by W. Minicozzi, who pointed out that the result also follows from the identity

$$
2 \int_{Z_{\lambda}}\left|\nabla_{g} e_{\lambda}\right|^{2} d S_{g}=-\int_{M} \operatorname{sgn}\left(\varphi_{\lambda}\right) \operatorname{div}_{g}\left(\left|\nabla_{g} e_{\lambda}\right| \nabla_{g} e_{\lambda}\right) d V_{g}
$$


This approach is used in $[\mathbf{A r}]$ to generalize the nodal bounds to Dirichlet and Neumann eigenfunctions of bounded domains.

Theorem 4.5 shows that Yau's conjectured lower bound would follow for a sequence of eigenfunctions satisfying $\left\|\varphi_{\lambda}\right\|_{L^{1}} \geq C>0$ for some positive constant $C$.

4.4. More general identities. It is possible to further generalize the identity of Proposition 4.2 and we pause to record an obvious one. For any function $\chi$, we have

$$
\Delta \chi(\varphi)=\chi^{\prime \prime}(\varphi)|\nabla \varphi|^{2}-\lambda^{2} \chi^{\prime}(\varphi) \varphi .
$$

We then take $\chi$ to be the meromorphic family of homogeneous distribution $x_{+}^{s}$. We recall that for $\operatorname{Re} a>-1$,

$$
x_{+}^{a}:=\left\{\begin{array}{cc}
x^{a}, & x \geq 0 \\
0, & x<0 .
\end{array}\right.
$$

The family extends to $a \in \mathbb{C}$ as a meromorphic family of distributions with simple poles at $a=-1,-2, \ldots,-k, \ldots$ using the equation $\frac{d}{d x} x_{+} s=s x_{+}^{s-1}$ to extend it one unit strip at a time. One can convert $x_{+}^{s}$ to the holomorphic family

$$
\chi_{+}^{\alpha}=\frac{x_{+}^{\alpha}}{\Gamma(\alpha+1)}, \quad \text { with } \chi_{+}^{-k}=\delta_{0}^{(k-1)}
$$

The identity we used above belongs to the family,

$$
\left(\Delta+s \lambda^{2}\right) \varphi_{+}^{s}=s(s-1)|\nabla \varphi|^{2} \varphi_{+}^{s-2} .
$$

Here $\varphi_{+}^{s}=\varphi^{*} x_{+}^{s}$ has poles at $s=-1,-2, \cdots$. The calculation in (21) used $|\varphi|$ but is equivalent to using (34) when $s=1$. Then $\varphi_{+}^{s-2}$ has a pole when $s=1$ with residue $\delta_{0}(\varphi)=\left.\frac{d S}{|\nabla \varphi|} d S\right|_{Z_{\varphi_{\lambda}}}$; it is cancelled by the factor $s-1$ and we obtain (21). This calculation is formal because the pullback formulae are only valid when $d \varphi \neq 0$ when $\varphi=0$, but as above they can be justified because the singular set has codimension 2 . The right side also has a pole at $s=0$ and we get $\Delta \varphi_{+}^{0}=-|\nabla \varphi|^{2} \delta^{\prime}(\varphi)$, which is equivalent to the divergence identity above. There are further poles at $s=-1,-2, \ldots$ but they now occur on both sides of the formulae. It is possible that they have further uses.

Such identities appear to be related to the Bernstein-Kashiwara theorem that for any real analytic function $f$ one may meromorphically extend $f_{+}^{s}$ to $\mathbb{C}$ by constructing a family $P_{s}(D)$ of differential operators with analytic coefficients and a meromorphic function $b(s)$ so that $P_{s}(D) f^{s+1}=b(s) f^{s}$. In the case $f=\varphi_{\lambda}$, the operator $|\nabla \varphi|^{-2}\left(\Delta+s \lambda^{2}\right)$ accomplishes something like this, although it does not have analytic coefficients due to poles at the critical points of $\varphi$. 
4.5. Other level sets. These results generalize easily to any level set $\mathcal{N}_{\varphi_{\lambda}}^{c}:=\left\{\varphi_{\lambda}=c\right\}$. Let $\operatorname{sgn}(x)=\frac{x}{|x|}$.

Proposition 4.6. For any $C^{\infty}$ Riemannian manifold, and any $f \in$ $C(M)$ we have,

$$
\int_{M} f\left(\Delta+\lambda^{2}\right)\left|\varphi_{\lambda}-c\right| d V+\lambda^{2} c \int f \operatorname{sgn}\left(\varphi_{\lambda}-c\right) d V=2 \int_{\mathcal{N}_{\varphi_{\lambda}}^{c}} f\left|\nabla \varphi_{\lambda}\right| d S
$$

This identity has similar implications for $\mathcal{H}^{n-1}\left(\mathcal{N}_{\varphi_{\lambda}}^{c}\right)$ and for the equidistribution of level sets. Note that if $c>\sup \left|\varphi_{\lambda}(x)\right|$ then indeed both sides are zero.

Corollary 4.7. For $c \in \mathbb{R}$

$$
\lambda^{2} \int_{\varphi_{\lambda} \geqslant c} \varphi_{\lambda} d V=\int_{\mathcal{N}_{\varphi_{\lambda}}^{c}}\left|\nabla \varphi_{\lambda}\right| d S \leq \lambda^{2} \operatorname{Vol}(M)^{1 / 2}
$$

Consequently, if $c>0$

$$
\mathcal{H}^{n-1}\left(\mathcal{N}_{\varphi_{\lambda}}^{c}\right)+\mathcal{H}^{n-1}\left(\mathcal{N}_{\varphi_{\lambda}}^{-c}\right) \geq C_{g} \lambda^{2-\frac{n+1}{2}} \int_{\left|\varphi_{\lambda}\right| \geq c}\left|\varphi_{\lambda}\right| d V .
$$

The Corollary follows by integrating $\Delta$ by parts, and by using the identity,

$$
\begin{aligned}
\int_{M}\left|\varphi_{\lambda}-c\right|+c \operatorname{sgn}\left(\varphi_{\lambda}-c\right) d V & =\int_{\varphi_{\lambda}>c} \varphi_{\lambda} d V-\int_{\varphi_{\lambda}<c} \varphi_{\lambda} d V \\
& =2 \int_{\varphi_{\lambda}>c} \varphi_{\lambda} d V
\end{aligned}
$$

since $0=\int_{M} \varphi_{\lambda} d V=\int_{\varphi_{\lambda}>c} \varphi_{\lambda} d V+\int_{\varphi_{\lambda}<c} \varphi_{\lambda} d V$.

4.6. Examples. The lower bound of Theorem 4.1 is far from the lower bound conjectured by Yau, which by Theorem 2.1 is correct at least in the real analytic case. In this section we go over the model examples to understand why the methds are not always getting sharp results.

4.7. Flat tori. We have, $|\nabla \sin \langle k, x\rangle|^{2}=\cos ^{2}\langle k, x\rangle|k|^{2}$. Since $\cos \langle k, x\rangle$ $=1$ when $\sin \langle k, x\rangle=0$ the integral is simply $|k|$ times the surface volume of the nodal set, which is known to be of size $|k|$. Also, we have $\int_{\mathbf{T}}|\sin \langle k, x\rangle| d x \geq C$. Thus, our method gives the sharp lower bound $\mathcal{H}^{n-1}\left(Z_{\varphi_{\lambda}}\right) \geq C \lambda^{1}$ in this example.

So the upper bound is achieved in this example. Also, we have $\int_{\mathbf{T}}|\sin \langle k, x\rangle| d x \geq C$. Thus, our method gives the sharp lower bound $\mathcal{H}^{n-1}\left(Z_{\varphi_{\lambda}}\right) \geq C \lambda^{1}$ in this example. Since $\cos \langle k, x\rangle=1$ when $\sin \langle k, x\rangle=0$ the integral is simply $|k|$ times the surface volume of the nodal set, which is known to be of size $|k|$. 
4.8. Spherical harmonics on $S^{2}$. The $L^{1}$ of $Y_{0}^{N}$ norm can be derived from the asymptotics of Legendre polynomials

$$
P_{N}(\cos \theta)=\sqrt{2}(\pi N \sin \theta)^{-\frac{1}{2}} \cos \left(\left(N+\frac{1}{2}\right) \theta-\frac{\pi}{4}\right)+O\left(N^{-3 / 2}\right)
$$

where the remainder is uniform on any interval $\epsilon<\theta<\pi-\epsilon$. We have

$$
\left\|Y_{0}^{N}\right\|_{L^{1}}=4 \pi \sqrt{\frac{(2 N+1)}{2 \pi}} \int_{0}^{\pi / 2}\left|P_{N}(\cos r)\right| d v(r) \sim C_{0}>0,
$$

i.e. the $L^{1}$ norm is asymptotically a positive constant. Hence $\int_{Z_{Y_{0}^{N}}}\left|\nabla Y_{0}^{N}\right| d s$ $\simeq C_{0} N^{2}$. In this example $\left|\nabla Y_{0}^{N}\right|_{L^{\infty}}=N^{\frac{3}{2}}$ saturates the sup norm bound. The length of the nodal line of $Y_{0}^{N}$ is of order $\lambda$, as one sees from the rotational invariance and by the fact that $P_{N}$ has $N$ zeros. The defect in the argument is that the bound $\left|\nabla Y_{0}^{N}\right|_{L^{\infty}}=N^{\frac{3}{2}}$ is only obtained on the nodal components near the poles, where each component has length $\simeq \frac{1}{N}$.

\section{Gaussian beams}

Gaussian beams are Gaussian shaped lumps which are concentrated on $\lambda^{-\frac{1}{2}}$ tubes $\mathcal{T}_{\lambda^{-\frac{1}{2}}}(\gamma)$ around closed geodesics and have height $\lambda^{\frac{n-1}{4}}$. We note that their $L^{1}$ norms decrease like $\lambda^{-\frac{(n-1)}{4}}$, i.e. they saturate the $L^{p}$ bounds of $[\mathbf{S o g}]$ for small $p$. In such cases we have $\int_{Z_{\varphi_{\lambda}}}\left|\nabla \varphi_{\lambda}\right| d S \simeq$ $\lambda^{2}\left\|\varphi_{\lambda}\right\|_{L^{1}} \simeq \lambda^{2-\frac{n-1}{4}}$. Gaussian beams are minimizers of the $L^{1}$ norm among $L^{2}$-normalized eigenfunctions of Riemannian manifolds. Also, the gradient bound $\left\|\nabla \varphi_{\lambda}\right\|_{L^{\infty}}=O\left(\lambda^{\frac{n+1}{2}}\right)$ is far off for Gaussian beams, the correct upper bound being $\lambda^{1+\frac{n-1}{4}}$. If we use these estimates on $\left\|\varphi_{\lambda}\right\|_{L^{1}}$ and $\left\|\nabla \varphi_{\lambda}\right\|_{L^{\infty}}$, our method gives $\mathcal{H}^{n-1}\left(Z_{\varphi_{\lambda}}\right) \geq C \lambda^{1-\frac{n-1}{2}}$, while $\lambda$ is the correct lower bound for Gaussian beams in the case of surfaces of revolution (or any real analytic case). The defect is again that the gradient estimate is achieved only very close to the closed geodesic of the Gaussian beam. Outside of the tube $\mathcal{T}_{\lambda^{-\frac{1}{2}}}(\gamma)$ of radius $\lambda^{-\frac{1}{2}}$ around the geodesic, the Gaussian beam and all of its derivatives decay like $e^{-\lambda d^{2}}$ where $d$ is the distance to the geodesic. Hence $\int_{Z_{\varphi_{\lambda}}}\left|\nabla \varphi_{\lambda}\right| d S \simeq \int_{Z_{\varphi_{\lambda}} \cap \mathcal{T}_{\lambda^{-\frac{1}{2}}}(\gamma)}\left|\nabla \varphi_{\lambda}\right| d S$. Applying the gradient bound for Gaussian beams to the latter integral gives $\mathcal{H}^{n-1}\left(Z_{\varphi_{\lambda}} \cap \mathcal{T}_{\lambda^{-\frac{1}{2}}}(\gamma)\right) \geq$ $C \lambda^{1-\frac{n-1}{2}}$, which is sharp since the intersection $Z_{\varphi_{\lambda}} \cap \mathcal{T}_{\lambda^{-\frac{1}{2}}}(\gamma)$ cuts across $\gamma$ in $\simeq \lambda$ equally spaced points (as one sees from the Gaussian beam approximation).

4.9. Non-scarring of nodal sets on $(M, g)$ with ergodic geodesic flow. The identity of Lemma 4.2 for general $f \in C^{2}(M)$ can be used to investigate the equidistribution of nodal sets equipped with the surface measure $\left|\nabla \varphi_{\lambda}\right| d S$. We denote the normalized measure by $\left.\lambda^{-2}\left|\nabla \varphi_{\lambda_{j}}\right| d S\right|_{Z_{\varphi_{\lambda}}}$. 
We first prove a rather simple (unpublished) result on nodal sets when the geodesic flow of $(M, g)$ is ergodic. Since there exist many expositions of quantum ergodic eigenfunctions, we only briefly recall the main facts and definitions and refer to $[\mathbf{Z 5}, \mathbf{Z 6}]$ for further background.

Quantum ergodicity concerns the semi-classical (large $\lambda$ ) asymptotics of eigenfunctions in the case where the geodesic flow $G^{t}$ of $(M, g)$ is ergodic. We recall that the geodesic flow is the Hamiltonian flow of the Hamiltonian $H(x, \xi)=|\xi|_{g}^{2}$ (the length squared) and that ergodicity means that the only $G^{t}$-invariant subsets of the unit cosphere bundle $S^{*} M$ have either full Liouville measure or zero Liouville measure (Liouville measure is the natural measure on the level set $H=1$ induced by the symplectic volume measure of $\left.T^{*} M\right)$.

We will say that a sequence $\left\{\varphi_{j_{k}}\right\}$ of $L^{2}$-normalized eigenfunctions is quantum ergodic if

$$
\left\langle A \varphi_{j_{k}}, \varphi_{j_{k}}\right\rangle \rightarrow \frac{1}{\mu\left(S^{*} M\right)} \int_{S^{*} M} \sigma_{A} d \mu, \quad \forall A \in \Psi^{0}(M) .
$$

Here, $\Psi^{s}(M)$ denotes the space of pseudodifferential operators of order $s$, $\sigma_{A}$ denotes the principal symbol of $A$, and $d \mu$ denotes Liouville measure on the unit cosphere bundle $S^{*} M$ of $(M, g)$. More generally, we denote by $d \mu_{r}$ the (surface) Liouville measure on $\partial B_{r}^{*} M$, defined by

$$
d \mu_{r}=\frac{\omega^{m}}{d|\xi|_{g}} \text { on } \partial B_{r}^{*} M
$$

We also denote by $\alpha$ the canonical action 1 -form of $T^{*} M$.

The main result is that there exists a subsequence $\left\{\varphi_{j_{k}}\right\}$ of eigenfunctions whose indices $j_{k}$ have counting density one for which $\rho_{j_{k}}(A):=$ $\left\langle A \varphi_{j_{k}}, \varphi_{j_{k}}\right\rangle \rightarrow \omega(A)$ (where as above $\omega(A)=\frac{1}{\mu\left(S^{*} M\right)} \int_{S^{*} M} \sigma_{A} d \mu$ is the normalized Liouville average of $\left.\sigma_{A}\right)$. The key quantities to study are the quantum variances

$$
V_{A}(\lambda):=\frac{1}{N(\lambda)} \sum_{j: \lambda_{j} \leq \lambda}\left|\left\langle A \varphi_{j}, \varphi_{j}\right\rangle-\omega(A)\right|^{2} .
$$

The following result is the culmination of the results in $[\mathbf{S h . 1}, \mathbf{Z 1}, \mathbf{C V}$, ZZw, GL].

THEOREM 4.8. Let $(M, g)$ be a compact Riemannian manifold (possibly with boundary), and let $\left\{\lambda_{j}, \varphi_{j}\right\}$ be the spectral data of its Laplacian $\Delta$. Then the geodesic flow $G^{t}$ is ergodic on $\left(S^{*} M, d \mu\right)$ if and only if, for every $A \in \Psi^{o}(M)$, we have:

(1) $\lim _{\lambda \rightarrow \infty} V_{A}(\lambda)=0$.

(2) $(\forall \epsilon)(\exists \delta) \lim \sup _{\lambda \rightarrow \infty} \frac{1}{N(\lambda)} \sum_{\substack{j \neq k: \lambda_{j}, \lambda_{k} \leq \lambda \\\left|\lambda_{j}-\lambda_{k}\right|<\delta}}\left|\left(A \varphi_{j}, \varphi_{k}\right)\right|^{2}<\epsilon$ 
Since all the terms in (1) are positive, no cancellation is possible, hence (1) is equivalent to the existence of a subset $\mathcal{S} \subset \mathbb{N}$ of density one such that $\mathcal{Q}_{\mathcal{S}}:=\left\{d \Phi_{k}: k \in \mathcal{S}\right\}$ has only $\omega$ as a weak* limit point.

We now consider nodal sets of quantum ergodic eigenfunctions. The following result says that if we equip nodal sets with the measure $\frac{1}{\lambda_{j}^{2}}\left|\nabla \varphi_{\lambda_{j}}\right| d S$, then nodal sets cannot 'scar', i.e. concentrate singularly as $\lambda_{j} \rightarrow \infty$.

Proposition 4.9. Suppose that $\left\{\varphi_{\lambda_{j}}\right\}$ is a quantum ergodic sequence. Then any weak limit of $\left\{\frac{1}{\lambda_{j}^{2}}\left|\nabla \varphi_{\lambda_{j}}\right| d S\right\}$ must be absolutely continuous with respect to $d V_{g}$.

(In fact this will be improved below in Corollary 4.13).

The first point is the following

Lemma 4.10. The weak * limits of the sequence $\left\{\left.\lambda^{-2}\left|\nabla \varphi_{\lambda_{j}}\right| d S\right|_{Z_{\varphi_{\lambda}}}\right\}$ of bounded positive measures are the same as the weak * limits of $\left\{\left|\varphi_{\lambda_{j}}\right|\right\}$ (against $f \in C(M)$.)

We let $f \in C^{2}(M)$ and multiply the identity of Proposition 4.2 by $\lambda^{-2}$. We then integrate by parts to put $\Delta$ on $f$. This shows that for $f \in C^{2}(M)$, we have

$$
\int_{M} f\left|\varphi_{\lambda}\right| d V=\lambda^{-2} \int_{Z_{\varphi_{\lambda}}} f\left|\nabla_{\lambda}\right| d S+O\left(\lambda^{-2}\right)
$$

Letting $f=1$, we see that the family of measures $\left\{\lambda^{-2}\left|\nabla \varphi_{\lambda_{j}}\right|^{2} \delta\left(\varphi_{\lambda_{j}}\right)\right\}$ is bounded. By uniform approximation of $f \in C(M)$ by elements of $C^{2}(M)$, we see that the weak* limit formula extends to $C(M)$.

Lemma 4.11. Suppose that $\left\{\varphi_{\lambda_{j}}\right\}$ is a quantum ergodic sequence. Then any weak limit of $\left\{\left|\varphi_{\lambda_{j}}\right| d S\right\}$ must be absolutely continuous with respect to $d V$.

We recall that a sequence of measures $\mu_{n}$ converges weak $*$ to $\mu$ if $\int_{M} f d \mu_{n} \rightarrow \int f d \mu$ for all continuous $f$. A basic fact about weak * convergence of measures is that $\int f d \mu_{n} \rightarrow \int f d \mu$ for all $f \in C(M)$ implies that $\mu_{n}(E) \rightarrow \mu(E)$ for all sets $E$ with $\mu(\partial E)=0$ (Portmanteau theorem).

We also recall that a sequence of eigenfunctions is called quantum ergodic (in the base) if

$$
\int f\left|\varphi_{\lambda_{j}}\right|^{2} d V \rightarrow \frac{1}{V o l(M)} \int_{M} f d V
$$

In other words, $\varphi_{\lambda}^{2} \rightarrow 1$ in the weak $*$ topology, i.e. the vague topology on measures. We now prove Lemma 4.11.

Proof. Suppose that $\left|\varphi_{\lambda_{j_{k}}}\right| d V \rightarrow d \mu$ and assume that $d \mu=c d V+d \nu$ where $d \nu$ is singular with respect to $d V$. Let $\Sigma=\operatorname{supp} \nu$, and let $\sigma=\mu(\Sigma)=\nu(\Sigma)$. Let $\mathcal{T}_{\epsilon}$ be the $\epsilon$-tube around $\Sigma$. Then

$$
\lim _{k \rightarrow \infty} \int_{\mathcal{T}_{\epsilon}}\left|\varphi_{\lambda_{j_{k}}}\right| d V=\operatorname{cVol}\left(T_{\epsilon}\right)+\nu(\Sigma)=\sigma+O(\epsilon)
$$


But for any set $\Omega \subset M, \int_{\Omega}\left|\varphi_{\lambda_{j}}\right| d V \leq \sqrt{\operatorname{Vol}(\Omega)} \sqrt{\int_{\Omega}\left|\varphi_{\lambda_{j}}\right|^{2} d V}$. Hence if $\operatorname{Vol}(\partial \Omega)=0, \limsup _{j \rightarrow \infty} \int_{\Omega}\left|\varphi_{\lambda_{j}}\right| d V \leq \operatorname{Vol}(\Omega)$. Letting $\Omega=\mathcal{T}_{\epsilon}(\Sigma)$ we get $\sigma+O(\epsilon) \leq \operatorname{Vol}\left(T_{\epsilon}(\Sigma)\right)=O(\epsilon)$ since $\lim _{k \rightarrow \infty} \int_{\mathcal{T}_{\epsilon}}\left|\varphi_{\lambda_{j_{k}}}\right|^{2} d V=\operatorname{Vol}\left(\mathcal{T}_{\epsilon}\right)=O(\epsilon)$. Letting $\epsilon \rightarrow 0$ gives a contradiction.

Of course, it is possible that the only weak* limit is zero.

4.10. A stronger non-scarring result. G. Rivière $[\mathbf{R i}]$ pointed out some improvements to Proposition 4.9.

Proposition 4.12. Suppose that $\left\{\varphi_{j_{k}}\right\}$ is a sequence of $L^{2}$ normalized eigenfunctions satisfying the following 'weak quantum ergodic' condition:

$(W Q E):\left|\varphi_{j_{k}}\right|^{2} d V_{g} \rightarrow \rho d V_{g}$ weak*, with $\rho \in L^{\infty}\left(M, d V_{g}\right)$.

Suppose also that

$$
\left|\varphi_{j_{k}}\right| d V_{g} \rightarrow d \mu
$$

where $\mu$ is a probability measure on $M$. Then $\mu=F d V_{g}$ with $F \in$ $L^{\infty}\left(M, d V_{g}\right)$.

In fact, the same is true for weak limits of $\left|\varphi_{j_{k}}\right|^{p} d V_{g}$ for any $1 \leq p<2$, but we only treat the case $p=1$.

Proof. If $f \in C(M)$ then

$$
\left|\int_{M} f\right| \varphi_{j_{k}}\left|d V_{g}\right| \leq\left.\left.\left|\int_{M}\left(|f|^{\frac{1}{2}}\left|\varphi_{j_{k}}\right|\right)\right| f\right|^{\frac{1}{2}} d V_{g}|\leq| \int_{M}|f|\left|\varphi_{j_{k}}\right|^{2} d V_{g}\right|^{\frac{1}{2}} \|\left. f\right|_{L^{1}} ^{\frac{1}{2}} .
$$

Let $k \rightarrow \infty$ and we get

$$
\left|\int_{M} f d \mu\right| \leq\|\rho\|_{L^{\infty}}\|f\|_{L^{1}}
$$

Hence $f \rightarrow \int f d \mu$ is a continuous linear functional on $L^{1}$ and must have the form $\mu=F d V_{g}$ where $\|F\|_{\infty} \leq\|\rho\|_{\infty}$.

Corollary 4.13. Suppose that $\left\{\varphi_{\lambda_{j}}\right\}$ is a quantum ergodic sequence. Then any weak limit of $\left\{\frac{1}{\lambda_{j}^{2}}\left|\nabla \varphi_{\lambda_{j}}\right| d S\right\}$ must be of the form $F d V_{g}$ with $|F| \leq 1$.

4.11. Weak* limits for $L^{\infty}$ quantum ergodic sequences. To our knowledge, the question whether the limit (4.8) holds $f \in L^{\infty}$ when $(M, g)$ has ergodic geodesic flow has not been studied. It is equivalent to strengthening the Portmanteau statement to all measurable sets $E$, and is equivalent to the statement that $\left\{\varphi_{\lambda_{j}}^{2}\right\} \rightarrow 1$ weakly in $L^{1}$. We call such sequences $L^{\infty}$ quantum ergodic on the base. The term 'on the base' refers to the fact that we only demand quantum ergodicity for the projections of the 'microlocal lifts' to the base $M$. For instance, the exponential eigenfunctions of flat tori are $L^{\infty}$ quantum ergodic in this sense. 
LEMma 4.14. Suppose that $\left\{\varphi_{j}\right\}$ is an $L^{\infty}$ - quantum ergodic sequence. Then there exists $\epsilon>0$ so that $\left\|\varphi_{j}\right\|_{L^{1}} \geq \epsilon>0$ for all $j$.

Proof. We argue by contradiction. If the conclusion were false, there would exist a subsequence $\varphi_{j_{k}} \rightarrow 0$ strongly in $L^{1}$, but with $\varphi_{j_{k}}^{2} d V \rightarrow d V$ weakly in $L^{1}$. The first assumption implies the existence of a subsequence (which we continue to denote by $\varphi_{j_{k}}$ ) satisfying $\varphi_{j_{k}} \rightarrow 0$ a.e. $d V$. But $L^{1}$ has the weak Banach-Saks property: any weakly convergent sequence in $L^{1}$ has a subsequence whose arithmetic means converge strongly (Szlenk's weak Banach-Saks theorem for $L^{1}$ ). We choose such a subsequence for $\varphi_{j_{k}}$ and continue to denote it as $\varphi_{j_{k}}$. This subsequence has the properties that

(1) $\varphi_{j_{k}} \rightarrow 0$ a.e.

(2) $\psi_{N}:=\frac{1}{N} \sum_{k \leq N} \varphi_{j_{k}}^{2} \rightarrow 1$ strongly in $L^{1}$.

But $\psi_{N}(x) \rightarrow 0$ on the same set where $\varphi_{j_{k}}(x) \rightarrow 0$, hence by $(1) \psi_{N} \rightarrow 0$ a.s. This contradicts (2) and completes the proof.

Combining with the above, we have

Corollary 4.15. Suppose that $\left\{\varphi_{\lambda_{j}}\right\}$ is an $L^{\infty}$ quantum ergodic sequence on the base. Then the conjectured Yau lower bound holds: $\mathcal{H}^{n-1}\left(Z_{\varphi_{\lambda}}\right)$ $\geq C_{g} \lambda$ for some $C_{g}>0$.

We also see that the limits in Proposition 4.9 are non-zero:

Corollary 4.16. Suppose that $\left\{\varphi_{\lambda_{j}}\right\}$ is an $L^{\infty}$ quantum ergodic sequence on the base. Then there exists $C>0$ so that any weak limit of the sequence $\left.\frac{1}{\lambda^{2}}\left|\nabla \varphi_{\lambda_{j}}\right| d S\right|_{Z_{\varphi_{\lambda_{j}}}}$ has mass $\geq C>0$.

Of course, such an abstract functional analysis argument only serves a purpose if we can prove that eigenfunctions of $\Delta$ are $L^{\infty}$ quantum ergodic on the base in interesting cases. It is natural to conjecture that this condition holds on negatively curved manifolds, since the expected $L^{1}$ norm of a random wave is bounded below by a positive constant. The main problem is that $L^{\infty}(M)$ is a non-separable Banach space. The standard quantum ergodicity arguments show that (when quantum ergodicity is valid), for any Borel set $E$ there exists a subsequence $\mathcal{S}_{E}$ of density one so that

$$
\lim _{k \rightarrow \infty, j_{k} \in \mathcal{S}_{E}} \int_{E} \varphi_{j_{k}}^{2} d V=\operatorname{Vol}(E) .
$$

However, the non-separability of $L^{\infty}(M)$ means that one cannot use the diagonalization argument of $[\mathbf{Z 1}, \mathbf{C V}]$ to show that there exists a density one subsequence independent of $E$ so that (41) holds. If $L^{\infty}$ quantum ergodicity fails, then zero-density subsequences of eigenfunctions would 'scar' along Cantor sets $\mathrm{C}$ of positive measure. That is, the mass $\int_{C} \varphi_{j_{k}}^{2} d V$ may tend to a larger value than $\operatorname{Vol}(C)$. 


\section{Equidistributed sums of Gaussian beams and quantum ergodicity}

We briefly consider the question whether it is possible to have a quantum ergodic sequence of eigenfunctions for which $\left\|\varphi_{j}\right\|_{L^{1}} \rightarrow 0$.

First, we observe that there do exist sequences of quantum ergodic functions (not eigenfunctions) with this property: $\sum_{j=1}^{M(n)} \sqrt{\frac{n}{M(n)}} \chi_{\left[x_{j}(n), x_{j}(n)+\frac{1}{n}\right]}$ $\rightarrow 0$ in $L^{1}([0,1], d x)$ as long as $M(n)=o(n)$. But its square is the probability measure $\frac{1}{M(n)} \sum_{j=1}^{M(n)} n \chi_{\left[x_{j}(n), x_{j}(n)+\frac{1}{n}\right]}$ and if the $\left\{x_{j}(n)\right\}$ are uniformly distribution in $[0,1]$ (w.r.t. $d x$ ), this tends weakly to $d x$.

It is tempting to construct sequences of eigenfunctions with the same property: a Gaussian beam $Y_{\gamma}^{N}$ on the standard $S^{2}$ associated to a closed geodesic $\gamma$ (i.e. a rotate of $Y_{N}^{N}$ ) is of height $\lambda^{\frac{1}{2}}$ in a tube of radius $\sqrt{\lambda}$ around $\gamma$. If we let $M(N)=o\left(N^{\frac{1}{2}}\right)$ and choose $M(N)$ closed geodesics which are $\frac{1}{\sqrt{M(N)}}$-separated, and become equidistributed in the space of closed geodesics, then $\varphi_{N}=\frac{1}{\sqrt{M(N)}} \sum_{j=1}^{M(N)} Y_{\gamma_{j}}^{N}$ is an eigenfunction whose $L^{1}$-norm tends to zero like $\sqrt{M(N)} N^{-\frac{1}{4}}$ but whose $L^{2}$ norm is asymptotic to 1 and whose modulus square tends weak ${ }^{*}$ to 1 . More precisely, $\frac{1}{M} \sum_{j=1}^{M(N)}\left|Y_{\gamma_{j}}^{N}\right|^{2} \rightarrow 1$ weakly. To prove that $\left|\varphi_{N}\right|^{2} \rightarrow 1$ requires proving that $\frac{1}{M(N)} \sum_{j \neq k} Y_{\gamma_{j}}^{N} \overline{Y_{\gamma_{k}}^{N}} \rightarrow 0$. The sum is over $\sim M(N)^{2}$ terms which are exponentially outside the tube intersections $T_{\lambda^{-\frac{1}{2}}}\left(\gamma_{j}\right) \cap T_{\lambda^{-\frac{1}{2}}}\left(\gamma_{k}\right)$. In the sum we may fix $j=j_{0}$ and multiply by $M(N)$. So we need then to show that $\sum_{k \neq j_{0}}\left|\left\langle Y_{\gamma_{j_{0}}}^{N}, Y_{\gamma_{k}}^{N}\right\rangle\right| \rightarrow 0$. The geodesics are well-separated if the distance in the space of geodesics between them is $\geq \frac{1}{\sqrt{M(N)}}$, which means that the angle between $\gamma_{j}$ and $\gamma_{k}$ is at least this amount. When the angle is $\geq \epsilon$ then the inner product $\left|\left\langle Y_{\gamma_{j}}^{N}, Y_{\gamma_{k}}^{N}\right\rangle\right| \leq \frac{1}{\epsilon} N^{-1}$ since the area of $T_{\lambda^{-\frac{1}{2}}}\left(\gamma_{j}\right) \cap T_{\lambda^{-\frac{1}{2}}}\left(\gamma_{k}\right)$ is bounded by this amount. For any $\epsilon$ the sum over geodesics separated by $\epsilon$ is $O\left(\frac{1}{\epsilon} M(N) N^{-1}\right)$. The remaining number of terms is $O\left(\epsilon^{2} M(N)\right)$. So if $\epsilon=o(\sqrt{M(N)})$ both terms tend to zero.

4.12. Intersections of nodal sets of orthogonal eigenfunctions. A related question is whether nodal sets of orthogonal eigenfunctions of the same eigenvalue must intersect. Of course, this question only arises when the eigenvalue has multiplicity $>1$. A result of this kind was obtained by V. Gichev under a topological condition on $M$.

ThEOREM 4.17. [Gi] Suppose that $H^{1}(M)=0$ and that $\varphi_{\lambda, 1}, \varphi_{\lambda, 2}$ are orthogonal eigenfunctions with the same eigenvalue $\lambda^{2}$. Then $Z_{\varphi_{\lambda, 1}} \cap Z_{\varphi_{\lambda, 2}} \neq$ $\emptyset$.

We briefly sketch the proof: Let $\mathcal{A}_{1}$ resp. $\mathcal{A}_{2}$ be the family of nodal domains of $\varphi_{\lambda, 1}$ resp. $\varphi_{\lambda, 2}$. Each union $\bigcup_{W \in \mathcal{A}_{j}} W$ covers $M$ up to the 
nodal set of $\varphi_{\lambda, j}$. If the nodal sets do not intersect then the nodal set of $\varphi_{\lambda, 2}$ is contained in $\bigcup_{W \in \mathcal{A}_{1}} W$, for instance; similarly if the indices are reversed. Hence the nodal sets have empty intersection if and only if $\bigcup_{W \in \mathcal{A}_{1}} W \cup \bigcup_{W \in \mathcal{A}_{2}} W$ covers $M$. Under this condition, Gichev constructs a closed 1-form which is not exact by showing that the incidence graph of the cover obtained from the union of the nodal domains of $\varphi_{\lambda, 1}$ and $\varphi_{\lambda, 2}$ contains a cycle. He then considers a nodal domain $U$ of $\varphi_{\lambda, 1}$ and a nodal domain $V$ of $\varphi_{\lambda, 2}$ which intersect. Let $Q=\partial U \cap V$. Since $Q \cap \partial V \neq \emptyset$ there exists a smooth function $f$ on $M$ such that $f \equiv 1$ in a neighborhood of $Q$ and $f=0$ near $\partial U \backslash Q$. Let $\eta$ be the one form which equals $d f$ on $U$ and 0 on the complement of $U$. Clearly $\eta$ is closed and it is verified in $[\mathbf{G i}]$ that $\eta$ is not exact.

Givech also proves that for $S^{2}$, if 0 is a regular value of $\varphi_{\lambda, 1}$ then $\# Z_{\varphi_{\lambda, 1}} \cap Z_{\varphi_{\lambda, 2}} \geq 2$ for every orthogonal eigenfunction $\varphi_{\lambda, 2}$ with the same eigenvalue. The proof is simply to use Green's formula for a nodal domain for $\varphi_{\lambda, 1}$ and note that the integral of $\varphi_{\lambda, 2} \frac{\partial}{\partial \nu} \varphi_{\lambda, 1}$ equals zero on its boundary.

A related observation is the curious identity of [SoZ], which holds for any $(M, g)$ : for any pair of eigenfunctions,

$$
\left(\lambda_{j}^{2}-\lambda_{k}^{2}\right) \int_{M} \varphi_{\lambda_{k}}\left|\varphi_{\lambda_{j}}\right| d V=2 \int_{Z_{\varphi_{\lambda_{j}}}} \varphi_{\lambda_{k}}\left|\nabla \varphi_{\lambda_{j}}\right| d S .
$$

Hence for a pair of orthogonal eigenfunctions of the same eigenvalue,

$$
\int_{Z_{\varphi_{\lambda_{j}}}} \varphi_{\lambda_{k}}\left|\nabla \varphi_{\lambda_{j}}\right| d S=0
$$

\section{Norms and nodal sets}

Studies of nodal sets often involve dual studies of $L^{p}$ norms of eigenfunctions. In this section, we review a number of relatively recent results on $L^{p}$ norms, both in the global manifold $M$ and for restrictions of eigenfunctions to submanifolds.

5.1. Polterovich-Sodin on norms and nodal sets. Let $\mathcal{A}\left(\varphi_{\lambda}\right)$ denote the collection of nodal domains of $\varphi_{\lambda}$. For $A \in \mathcal{A}\left(\varphi_{\lambda}\right)$ let $m_{A}=$ $\max _{A}\left|\varphi_{\lambda}\right|$. In [PS] the following is proved (see Corollary 1.7):

Theorem 5.1. [PS] Let $(M, g)$ be a $C^{\infty}$ Riemannian surface. For every $\varphi_{\lambda}$ with $\left\|\varphi_{\lambda}\right\|=1$,

$$
\sum_{A \in \mathcal{A}} m_{A}^{6} \leq k_{g} \lambda^{3}
$$

Hence, for each $a>0$, the number of nodal domains $A$ of $\varphi_{\lambda}$ where the maximal bound $m_{A} \geq a \lambda^{1 / 2}$ is achieved in order of magnitude does not exceed $k_{g} a^{-6}$. In particular, for fixed a, it remains bounded as $\lambda \rightarrow \infty$. 
The proof uses a certain Bananch indicatrix, the Sogge $L^{6}$ bounds, and estimates on the inradius of nodal domains. For a continuous function $u \in C(\mathbb{R})$, the generalized Banach indicatrix is defined by

$$
B(u, f)=\int_{-\infty}^{+\infty} u(c) \beta(c, f) d c
$$

where for a regular value $c \in \mathbb{R}$ of $f, \beta(c, f)$ is the number of connected components of $f^{-1}(c)$. In [PS], the integral $\left.B(u, f)\right)$ is bounded from above through the $L^{2}$-norms of the function $f$ and $\Delta f$. I.e.. in Theorem 1.3. For any $f \in \mathcal{F}_{\lambda}$ and any continuous function $u$ on $\mathbb{R}$,

$$
B(u, f) \leq k_{g}\|u \circ f\|(\|f\|+\|\Delta f\|) .
$$

The proof is roughly as follows: Let $p_{i}$ be a point of $A_{i}$ where the maximum is achieved. By the inradius bound [Man3], there exists $\mu>0$ so that the disc $D\left(p_{j}, \frac{\mu}{\lambda}\right) \subset A_{i}$. One can then express $\varphi_{\lambda}$ in $D\left(p_{j}, \frac{\mu}{\lambda}\right)$ by the sum of a Green's integral and Poisson integral with respect to the Euclidean Dirichlet Green's function of a slightly smaller disc. In particular one may express $\varphi_{\lambda}\left(p_{j}\right)$ by such an integral. Apply Hölder's inequality one gets

$$
m_{j}^{6} \leq k_{g} \lambda^{2} \int_{D\left(p_{j}, r\right)} \varphi_{\lambda}^{6} d V, \quad\left(r=\mu \lambda^{-\frac{1}{2}}\right) .
$$

Since the discs are disjoint one can sum in $j$ and apply the Sogge $L^{6}$ bound to include the proof. Thus, the only fact one used about nodal domains was lower bound on the inradius.

This result bears a curious comparison to the results of $[\mathbf{S T Z}]$ giving new constraints on $(M, g)$ which are of maximal eigenfunction growth, i.e. possess eigenfunctions such that $m_{A} \geq C \lambda^{\frac{1}{2}}$ for some sequence of eigenfunctions $\varphi_{\lambda_{j}}$ with $\lambda_{j} \rightarrow \infty$. The result (building on older results of Sogge and the author) states that such a sequence can exist only if $(M, g)$ possesses a 'pole' $p$ for which the set of geodesic loops $\mathcal{L}_{p}$ based at $p$ has positive measure in $S_{p}^{*} M$ (with respect to the natural spherical volume measure) and such that the first return map has a recurrence property. In fact, the only known surfaces where the bounds are achieved are surfaces of revolution, and in this case the first return map is the identity. It is quite plausible that if $(M, g)$ has maximal eigenfunction growth, then the first return map must be the identity map on a set of positive measure in $\mathcal{L}_{p}$.

Combined with the Polterovich-Sodin result above, we see that such 'poles' $p$, when they exist, can only occur in a uniformly bounded number of nodal domains of a surface. It would be interesting to know if there can exist only a finite number of such points at all if one additionally assumes that the set of smoothly closed geodesics has measure zero. For instance, in that case, there might be a unique pole in each of the finite number of possible nodal domains. This finitude problem would be useful in strengthening the condition on $(M, g)$ of maximal eigenfunction growth. 
5.2. Norms of restrictions. A problem of current interest is to consider $L^{p}$ norms of restrictions of eigenfunctions to hypersurfaces or higher codimension submanifolds. For expository purposes we only consider geodesics on surfaces here. Following earlier work of A. Reznikov, Burq, Gérard and Tzvetkov [BGT] proved

THEOREM 5.2. [BGT] Suppose that $(M, g)$ is a compact surface, then there exists $\lambda_{0}(\epsilon), C>0$ so that, for any geodesic segment $\gamma$ of length $L_{\gamma}$ and any eigenfunction $\varphi_{\lambda}$ with $\lambda \geq \lambda_{0}$ we have

$$
\frac{1}{L_{\gamma}} \int_{\gamma}\left|\varphi_{\lambda}\right|^{2} d s \leq C \lambda^{\frac{1}{2}}\left\|\varphi_{\lambda}\right\|^{2}
$$

Their estimate is sharp for the round sphere $S^{2}$ because of the highest weight spherical harmonics They also showed that for all geodesic segments $\gamma$ of unit length,

$$
\left(\frac{1}{L_{\gamma}} \int_{\gamma}\left|\varphi_{\lambda}\right|^{4} d s\right)^{1 / 4} \leqslant C \lambda^{\frac{1}{4}}\left\|e_{\lambda}\right\|_{L^{2}(M)},
$$

The estimate is only known to be achieved when the geodesic is elliptic, and quite likely it can be improved if the geodesic is hyperbolic. A result in this direction is:

TheOrem 5.3. [SoZ2] Suppose that $(M, g)$ is a compact surface of nonpositive curvature. Then for all $\epsilon$, there exists $\lambda_{0}(\epsilon), C>0$ so that, for any geodesic segment $\gamma$ of length $L_{\gamma}$ and any eigenfunction $\varphi_{\lambda}$ with $\lambda \geq \lambda_{0}(\epsilon)$, we have

$$
\frac{1}{L_{\gamma}} \int_{\gamma}\left|\varphi_{\lambda}\right|^{2} d s \leq C \epsilon \lambda^{\frac{1}{2}}\left\|\varphi_{\lambda}\right\|^{2}
$$

A related result on $L^{4}$ norms is,

Theorem 5.4. [SoZ3] Let $(M, g)$ be a surface and assume that the set

$$
\mathcal{P}=\left\{(x, \xi) \in S^{*} M: g^{t}(x, \xi)=(x, \xi), \text { some } t>0\right\}
$$

of periodic points has Liouville measure zero in $S^{*} M$. Then there is a subsequence of eigenvalues $\lambda_{j_{k}}$ of density one so that

$$
\left\|e_{\lambda_{j_{k}}}\right\|_{L^{4}(M)}=o\left(\lambda_{j_{k}}^{1 / 8}\right) \text {. }
$$

The results are based in part on a relatively new Kakeya-Nikodym maximal function estimate of Bourgain [Bourg], as improved by Sogge [Sog2]. We believe that it can be improved the following phase space Kakeya-Nikodym theorem. Let $T_{\delta}(\gamma)$ be the tube of radius $\delta$ around a geodesic arc in $M$, and let $\chi_{\delta, \gamma}$ be a smooth cutoff to a phase space tube of its lift to $S^{*} M$. Then for all $\epsilon$, there exists $\delta(\epsilon)$ such that

$$
\limsup _{\lambda \rightarrow \infty} \frac{1}{N(\lambda)} \sum_{\lambda_{j} \leqslant \lambda} \sup _{\gamma \in \Pi} \int_{T_{\delta(\epsilon)}(\gamma)}\left|\varphi_{\lambda}\right|^{2} d s<\epsilon .
$$


We expect the sup occurs when $\gamma$ is the orbit of $(x, \xi)$. But then it is easy to estimate the right side and one should be able to get a quantitative improvement of Theorem 5.4.

5.3. Quantum ergodic restriction (QER) theorems. In this section we briefly review a recent series of results [TZ2, TZ3, DZ, CTZ] on quantum ergodic restriction theorems. They are used in section $\S 10$ to determine the limit distribution of intersections of nodal lines and geodesics on real analytic surfaces (in the complex domain).

Let $H \subset M$ be a hypersurface and consider the Cauchy data $\left(\left.\varphi_{j}\right|_{H},\left.\lambda_{j}^{-1} \partial_{\nu} \varphi_{j}\right|_{H}\right)$ of eigenfunctions along $H$; here $\partial_{\nu}$ is the normal derivative. We refer to $\left.\varphi_{j}\right|_{H}$ as the Dirichlet data and to $\left.\lambda_{j}^{-1} \partial_{\nu} \varphi_{j}\right|_{H}$ as the Neumann data. A QER (quantum ergodic restriction) theorem seeks to find limits of matrix elements of this data along $H$ with respect to pseudodifferential operators $O p_{H}(a)$ on $H$. The main idea is that $S_{H}^{*} M$, the set of unit covectors with footpoints on $H$, is a cross-section to the geodesic flow and the first return map of the geodesic flow for $S_{H}^{*} M$ is ergodic. The Cauchy data should be the quantum analogue of such a cross section and therefore should be quantum ergodic on $H$.

For applications to nodal sets and other problems, it is important to know if the Dirichlet data alone satisfies a QER theorem. The answer is obviously 'no' in general. For instance if $(M, g)$ has an isometric involution and with a hypersurface $H$ of fixed points, then any eigenfunction which is odd with respect to the involution vanishes on $H$. But in [TZ2, TZ3] a sufficient condition is given for quantum ergodic restriction, which rules out this and more general situations. The symmetry condition is that geodesics emanating from the 'left side' of $H$ have a different return map from geodesics on the 'right side' when the initial conditions are reflections of each other through $T H$. To take the simplest example of the circle, the restriction of $\sin k x$ to a point is never quantum ergodic but the full Cauchy data $(\cos k x, \sin k x)$ of course satisfies $\cos ^{2} k x+\sin ^{2} k x=1$. In [CTZ] it is proved that Cauchy data always satisfies QER for any hypersurface. This has implications for (at least complex) zeros of even or odd eigenfunctions along an axis of symmetry, e.g. for the case of Maass forms for the modular domain $S L(2, \mathbb{Z}) / \mathcal{H}^{2}$ (see $\left.\S 10\right)$.

To state the QER theorem, we introduce some notation. We put

$$
T_{H}^{*} M=\left\{(q, \xi) \in T_{q}^{*} M, \quad q \in H\right\}, \quad T^{*} H=\left\{(q, \eta) \in T_{q}^{*} H, \quad q \in H\right\} .
$$

We further denote by $\pi_{H}: T_{H}^{*} M \rightarrow T^{*} H$ the restriction map,

$$
\pi_{H}(x, \xi)=\left.\xi\right|_{T H} .
$$

For any orientable (embedded) hypersurface $H \subset M$, there exists two unit normal co-vector fields $\nu_{ \pm}$to $H$ which span half ray bundles $N_{ \pm}=$ $\mathbb{R}_{+} \nu_{ \pm} \subset N^{*} H$. Infinitesimally, they define two 'sides' of $H$, indeed they are the two components of $T_{H}^{*} M \backslash T^{*} H$. We use Fermi normal coordinates $\left(s, y_{n}\right)$ 
along $H$ with $s \in H$ and with $x=\exp _{x} y_{n} \nu$ and let $\sigma, \eta_{n}$ denote the dual symplectic coordinates. For $(s, \sigma) \in B^{*} H$ (the co-ball bundle), there exist two unit covectors $\xi_{ \pm}(s, \sigma) \in S_{s}^{*} M$ such that $\left|\xi_{ \pm}(s, \sigma)\right|=1$ and $\left.\xi\right|_{T_{s} H}=\sigma$. In the above orthogonal decomposition, they are given by

$$
\xi_{ \pm}(s, \sigma)=\sigma \pm \sqrt{1-|\sigma|^{2}} \nu_{+}(s) .
$$

We define the reflection involution through $T^{*} H$ by

$$
r_{H}: T_{H}^{*} M \rightarrow T_{H}^{*} M, \quad r_{H}\left(s, \mu \xi_{ \pm}(s, \sigma)\right)=\left(s, \mu \xi_{\mp}(s, \sigma)\right), \mu \in \mathbb{R}_{+} .
$$

Its fixed point set is $T^{*} H$.

We denote by $G^{t}$ the homogeneous geodesic flow of $(M, g)$, i.e. Hamiltonian flow on $T^{*} M-0$ generated by $|\xi|_{g}$. We define the first return time $T(s, \xi)$ on $S_{H}^{*} M$ by,

$$
\left.T(s, \xi)=\inf \left\{t>0: G^{t}(s, \xi) \in S_{H}^{*} M, \quad(s, \xi) \in S_{H}^{*} M\right)\right\} .
$$

By definition $T(s, \xi)=+\infty$ if the trajectory through $(s, \xi)$ fails to return to $H$. Inductively, we define the jth return time $T^{(j)}(s, \xi)$ to $S_{H}^{*} M$ and the jth return map $\Phi^{j}$ when the return times are finite.

We define the first return map on the same domain by

$$
\Phi: S_{H}^{*} M \rightarrow S_{H}^{*} M, \quad \Phi(s, \xi)=G^{T(s, \xi)}(s, \xi)
$$

When $G^{t}$ is ergodic, $\Phi$ is defined almost everywhere and is also ergodic with respect to Liouville measure $\mu_{L, H}$ on $S_{H}^{*} M$.

Definition: We say that $H$ has a positive measure of microlocal reflection symmetry if

$$
\mu_{L, H}\left(\bigcup_{j \neq 0}^{\infty}\left\{(s, \xi) \in S_{H}^{*} M: r_{H} G^{T^{(j)}(s, \xi)}(s, \xi)=G^{T^{(j)}(s, \xi)} r_{H}(s, \xi)\right\}\right)>0 .
$$

Otherwise we say that $H$ is asymmetric with respect to the geodesic flow.

The QER theorem we state below holds for both poly-homogeneous (Kohn-Nirenberg) pseudo-differential operators as in [HoI-IV] and also for semi-classical pseudo-differential operators on $H[\mathbf{Z w}]$ with essentially the same proof. To avoid confusion between pseudodifferential operators on the ambient manifold $M$ and those on $H$, we denote the latter by $O p_{H}(a)$ where $a \in S_{c l}^{0}\left(T^{*} H\right)$. By Kohn-Nirenberg pseudo-differential operators we mean operators with classical poly-homogeneous symbols $a(s, \sigma) \in C^{\infty}\left(T^{*} H\right)$,

$$
a(s, \sigma) \sim \sum_{k=0}^{\infty} a_{-k}(s, \sigma),\left(a_{-k} \text { positive homogeneous of order }-k\right)
$$

as $|\sigma| \rightarrow \infty$ on $T^{*} H$ as in [HoI-IV]. By semi-classical pseudo-differential operators we mean $h$-quantizations of semi-classical symbols $a \in S^{0,0}\left(T^{*} H \times\right.$ 
$\left.\left(0, h_{0}\right]\right)$ of the form

$$
a_{h}(s, \sigma) \sim \sum_{k=0}^{\infty} h^{k} a_{-k}(s, \sigma),\left(a_{-k} \in S_{1,0}^{0}\left(T^{*} H\right)\right)
$$

as in $[\mathbf{Z w}, \mathbf{H Z}, \mathbf{T Z}]$.

We further introduce the zeroth order homogeneous function

$$
\gamma\left(s, y_{n}, \sigma, \eta_{n}\right)=\frac{\left|\eta_{n}\right|}{\sqrt{|\sigma|^{2}+\left|\eta_{n}\right|^{2}}}=\left(1-\frac{|\sigma|^{2}}{r^{2}}\right)^{\frac{1}{2}}, \quad\left(r^{2}=|\sigma|^{2}+\left|\eta_{n}\right|^{2}\right)
$$

on $T_{H}^{*} M$ and also denote by

$$
\gamma_{B^{*} H}=\left(1-|\sigma|^{2}\right)^{\frac{1}{2}}
$$

its restriction to $S_{H}^{*} M=\{r=1\}$.

For homogeneous pseudo-differential operators, the QER theorem is as follows:

TheOREM 5.5. [TZ, TZ2, DZ] Let $(M, g)$ be a compact manifold with ergodic geodesic flow, and let $H \subset M$ be a hypersurface. Let $\varphi_{\lambda_{j}} ; j=1,2, \ldots$ denote the $L^{2}$-normalized eigenfunctions of $\Delta_{g}$. If $H$ has a zero measure of microlocal symmetry, then there exists a density-one subset $S$ of $\mathbb{N}$ such that for $\lambda_{0}>0$ and $a(s, \sigma) \in S_{c l}^{0}\left(T^{*} H\right)$

$$
\lim _{\lambda_{j} \rightarrow \infty ; j \in S}\left\langle O p_{H}(a) \gamma_{H} \varphi_{\lambda_{j}}, \gamma_{H} \varphi_{\lambda_{j}}\right\rangle_{L^{2}(H)}=\omega(a),
$$

where

$$
\omega(a)=\frac{2}{\operatorname{vol}\left(S^{*} M\right)} \int_{B^{*} H} a_{0}(s, \sigma) \gamma_{B^{*} H}^{-1}(s, \sigma) d s d \sigma .
$$

Alternatively, one can write $\omega(a)=\frac{1}{\operatorname{vol}\left(S^{*} M\right)} \int_{S_{H}^{*} M} a_{0}\left(s, \pi_{H}(\xi)\right) d \mu_{L, H}(\xi)$. Note that $a_{0}(s, \sigma)$ is bounded but is not defined for $\sigma=0$, hence $a_{0}\left(s, \pi_{H}(\xi)\right)$ is not defined for $\xi \in N^{*} H$ if $a_{0}(s, \sigma)$ is homogeneous of order zero on $T^{*} H$. The analogous result for semi-classical pseudo-differential operators is:

TheOrem 5.6. [TZ, TZ2, DZ] Let $(M, g)$ be a compact manifold with ergodic geodesic flow, and let $H \subset M$ be a hypersurface. If $H$ has a zero measure of microlocal symmetry, then there exists a density-one subset $S$ of $\mathbb{N}$ such that for $a \in S^{0,0}\left(T^{*} H \times\left[0, h_{0}\right)\right)$,

$$
\lim _{h_{j} \rightarrow 0^{+} ; j \in S}\left\langle O p_{h_{j}}(a) \gamma_{H} \varphi_{h_{j}}, \gamma_{H} \varphi_{h_{j}}\right\rangle_{L^{2}(H)}=\omega(a),
$$

where

$$
\omega(a)=\frac{2}{\operatorname{vol}\left(S^{*} M\right)} \int_{B^{*} H} a_{0}(s, \sigma) \gamma_{B^{*} H}^{-1}(s, \sigma) d s d \sigma .
$$

Examples of asymmetric curves on surfaces in the case where $(M, g)$ is a finite area hyperbolic surface are the following:

- $H$ is a geodesic circle; 
- $H$ is a closed horocycle of radius $r<\operatorname{inj}(M, g)$, the injectivity radius.

- $H$ is a generic closed geodesic or an arc of a generic non-closed geodesic.

\section{Critical points}

In this section, we briefly discuss some analogues of (16) and (21) for critical points on surfaces. To be sure, it is not hard to generate many identities; the main problem is to derive information from them.

We denote the gradient of a function $\varphi$ by $\nabla \varphi$ and its Hessian by $\nabla^{2} \varphi:=\nabla d \varphi$, where $\nabla$ is the Riemannian connection. We also denote the area form by $d A$ and the scalar curvature by $K$. The results are based on unpublished work in progress of the author. It is often said that measuring critical point sets and values is much more difficult than measuring nodal sets; the identities reflect this difficulty in that the identities become signed:

Proposition 6.1. Suppose that $(M, g)$ is a Riemannian surface, and that $\varphi$ is a Morse eigenfunction with $\left(\Delta+\lambda^{2}\right) \varphi=0$. Let $V \in C^{2}(M)$. Then

$$
\begin{aligned}
2 \pi \sum_{p: d \varphi(p)=0} \operatorname{sign}\left(\operatorname{det} \nabla^{2} \varphi(p)\right) V(p)= & 2 \lambda^{2} \int_{M} \frac{\varphi}{|\nabla \varphi|} \frac{\nabla V \cdot \nabla \varphi}{|\nabla \varphi|} d A+2 \int_{M} K V d A \\
& -\int_{M}(\Delta V) \log |\nabla \varphi|^{2} d A .
\end{aligned}
$$

Here, $\operatorname{sign}\left(\operatorname{det} \nabla^{2} \varphi(p)\right)=1$ if $p$ is a local maximum or minimum and $=-1$ if $p$ is a saddle point. When $V \equiv 1$, the identity reduces to the Gauss-Bonnet theorem $\int K d A=2 \pi \chi(M)$ and the Hopf index formula $\chi(M)=\sum_{x: \nabla \varphi(x)=0} \operatorname{sign}\left(\operatorname{det} \nabla^{2} \varphi(p)\right)$. As this indicates, the main problem with applying the identity to counting critical points is that the left side is an alternating sum over critical points rather than a positive sum. In [Dong] a related identity using $|\nabla \varphi|^{2}+\lambda^{2} \varphi^{2}$ produced a sum of constant sign over the singular points of $\varphi$, but singular points are always saddle points of index -1 and hence of constant sign. Note that under the Morse assumption, $\log |\nabla \varphi|,|\nabla \varphi|^{-1} \in L^{1}(M, d A)$, so that the right side is a well defined measure integrated against $V$.

We now make some interesting choices of $V$. As mentioned above, (weighted) counting of critical values should be simpler than weighted counting of critical points. Hence we put $V=f(\varphi)$ for smooth $f$. This choice does give cancellation of the 'bad factor' $|\nabla \varphi|^{-1}$ and (using that $\left.\Delta f(\varphi)=f^{\prime \prime}(\varphi)|\nabla \varphi|^{2}-f^{\prime}(\varphi) \lambda^{2} \varphi\right)$ we get 
Corollary 1. With the assumptions of Proposition 6.1, if $f \in C^{2}(\mathbb{R})$, then

$$
\begin{aligned}
2 \pi \sum_{p: d \varphi(p)=0} \operatorname{sign}\left(\operatorname{det} \nabla^{2} \varphi(p)\right) f(\varphi(p))= & 2 \lambda^{2} \int_{M} \varphi f^{\prime}(\varphi) d A+2 \int_{M} K f(\varphi) d A \\
& \left.-\int_{M}\left(f^{\prime \prime}(\varphi)|\nabla \varphi|^{2}-f^{\prime}(\varphi) \lambda^{2} \varphi\right)\right) \log |\nabla \varphi|^{2} d A .
\end{aligned}
$$

Of course, this still has the defect that the left side is an oscillating sum, and the factor $f(\varphi)$ in the sum damps out the critical points in regions of exponential decay. To illustrate, if $f(x)=x$ we get

$$
2 \pi \sum_{p: d \varphi(p)=0} \operatorname{sign}\left(\operatorname{det} \nabla^{2} \varphi(p)\right) \varphi(p)=2 \int_{M} K \varphi d A+\lambda^{2} \int_{M} \varphi \log |\nabla \varphi|^{2} d A .
$$

To highlight the sign issue, we break up the sum into the sub-sum over maxima/minima and the sub-sum over saddle points, denoting the set of local maxima (resp. minima) by $\max$ (resp. min) and the set of saddle

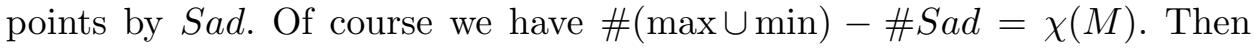
(55) is equivalent to

$$
\begin{aligned}
2 \pi \sum_{p \in \max \cup \min } f(\varphi(p))= & 2 \pi \sum_{p \in S a d} f(\varphi(p))+2 \lambda^{2} \int_{M} \varphi f^{\prime}(\varphi) d A \\
& +2 \int_{M} K f(\varphi) d A-\int_{M}\left(f^{\prime \prime}(\varphi)|\nabla \varphi|^{2}\right. \\
& \left.\left.-f^{\prime}(\varphi) \lambda^{2} \varphi\right)\right) \log |\nabla \varphi|^{2} d A .
\end{aligned}
$$

We write $\log r=\log _{+} r-\log _{-} r$ where $\log _{+} r=\max \{\log r, 0\}$. We note that on any compact Riemannian manifold, $\log _{+}|\nabla \varphi|^{2}=O(\log \lambda)$ uniformly in $x$ as $\lambda \rightarrow \infty$ while $\log _{-}|\nabla \varphi|^{2}$ can be quite complicated to estimate. When $f=x^{2}$ we get,

$$
\begin{aligned}
2 \pi \sum_{p \in \max , \min } \varphi^{2}(p)= & 2 \pi \sum_{p \in \text { Sad }} \varphi(p)^{2}+4 \lambda^{2} \\
& +2 \int_{M}\left(\lambda^{2} \varphi^{2}-|\nabla \varphi|^{2}\right) \log |\nabla \varphi|^{2} d A+2 \int_{M} K \varphi^{2} d A .
\end{aligned}
$$

Assuming $\varphi$ is a Morse eigenfunction, this implies

$$
\sum_{p \in \max , \min } \varphi^{2}(p) \leq \sum_{p \in \text { Sad }} \varphi(p)^{2}+O\left(\lambda^{2} \log \lambda\right) .
$$

To get rid of the signs in the sum, we could choose $V=W \operatorname{det} \nabla^{2} \varphi$, where the determinant is defined by the metric. Since $\left(\operatorname{sign} \operatorname{det} \nabla^{2} \varphi\right) \operatorname{det} \nabla^{2} \varphi=$ 
$\left|\operatorname{det} \nabla^{2} \varphi\right|$ we obtain

$$
\begin{aligned}
\left.2 \pi \sum_{p: d \varphi(p)=0}\left|\operatorname{det} \nabla^{2} \varphi(p)\right| W(p)\right)= & 2 \lambda^{2} \int_{M} \frac{\varphi}{|\nabla \varphi|} \frac{\nabla\left(W \operatorname{det} \nabla^{2} \varphi\right) \cdot \nabla \varphi}{|\nabla \varphi|} d A \\
& +2 \int_{M} K W \operatorname{det} \nabla^{2} \varphi d A \\
& -\int_{M}\left(\Delta W \operatorname{det} \nabla^{2} \varphi\right) \log |\nabla \varphi|^{2} d A
\end{aligned}
$$

But the first term appears to be difficult to estimate.

The optimist might conjecture the following Bézout bound for the number of critical values of eigenfunctions in the real analytic case:

Conjecture 6.2. If $(M, g)$ is real analytic then the number $\# C V\left(\varphi_{\lambda}\right)$ of critical values of $\varphi_{\lambda}$ satisfies $\# C V\left(\varphi_{\lambda}\right) \leq C_{g} \lambda^{n}($ where $n=\operatorname{dim} M)$.

Note that the critical point set could have codimension 1 (e.g. rotationally invariant eigenfunctions on a surface of revolution), so that in general we cannot count critical points. The number of critical values is generically the same as the number of connected components, although there could exist high multiplicities in the number of components of a give critical level.

The conjecture is motivated by Bézout's theorem for the number of intersection points of $n$ real algebraic varieties of degree $\lambda$ in dimension $n$. But it is difficult to control intersections in the real analytic case and it is not very clear at present how plausible the conjecture is.

\section{Analytic continuation of eigenfunctions for real analytic $(M, g)$}

We now take up the theme mentioned in the introduction of analytically continuing eigenfunctions on real analytic $(M, g)$ to the complex domain. In the next sections we apply the analytic continuation to the study of nodal of eigenfunctions in the real analytic case. For background we refer to [LS1, LS2, GS1, GS2, GLS, Z8].

A real analytic manifold $M$ always possesses a unique complexification $M_{\mathbb{C}}$ generalizing the complexification of $\mathbb{R}^{m}$ as $\mathbb{C}^{m}$. The complexification is an open complex manifold in which $M$ embeds $\iota: M \rightarrow M_{\mathbb{C}}$ as a totally real submanifold (Bruhat-Whitney). As examples, we have:

- $M=\mathbb{R}^{m} / \mathbb{Z}^{m}$ is $M_{\mathbb{C}}=\mathbb{C}^{m} / \mathbb{Z}^{m}$.

- The unit sphere $S^{n}$ defined by $x_{1}^{2}+\cdots+x_{n+1}^{2}=1$ in $\mathbb{R}^{n+1}$ is complexified as the complex quadric $S_{\mathbb{C}}^{2}=\left\{\left(z_{1}, \ldots, z_{n}\right) \in \mathbb{C}^{n+1}\right.$ : $\left.z_{1}^{2}+\cdots+z_{n+1}^{2}=1\right\}$.

- The hyperboloid model of hyperbolic space is the hypersurface in $\mathbb{R}^{n+1}$ defined by

$$
\mathbb{H}^{n}=\left\{x_{1}^{2}+\cdots x_{n}^{2}-x_{n+1}^{2}=-1, \quad x_{n}>0\right\} .
$$

Then,

$$
H_{\mathbb{C}}^{n}=\left\{\left(z_{1}, \ldots, z_{n+1}\right) \in \mathbb{C}^{n+1}: z_{1}^{2}+\cdots z_{n}^{2}-z_{n+1}^{2}=-1\right\} .
$$


- Any real algebraic subvariety of $\mathbb{R}^{m}$ has a similar complexification.

- Any Lie group $G$ (or symmetric space) admits a complexification $G_{\mathbb{C}}$.

The Riemannian metric determines a special kind of distance function on $M_{\mathbb{C}}$ known as a Grauert tube function. It is the plurisubharmonic function $\sqrt{\rho}=\sqrt{\rho}_{g}$ on $M_{\mathbb{C}}$ defined as the unique solution of the Monge-Ampère equation

$$
(\partial \bar{\partial} \sqrt{\rho})^{m}=\delta_{M_{\mathbb{R}}, d V_{g}}, \quad \iota^{*}(i \partial \bar{\partial} \rho)=g .
$$

Here, $\delta_{M_{\mathbb{R}}, d V_{g}}$ is the delta-function on the real $M$ with respect to the volume form $d V_{g}$, i.e. $f \rightarrow \int_{M} f d V_{g}$. In fact, it is observed in [GS1, GLS] that the Grauert tube function is obtained from the distance function by setting $\sqrt{\rho}(\zeta)=i \sqrt{r^{2}(\zeta, \bar{\zeta})}$ where $r^{2}(x, y)$ is the squared distance function in a neighborhood of the diagonal in $M \times M$.

One defines the Grauert tubes $M_{\tau}=\left\{\zeta \in M_{\mathbb{C}}: \sqrt{\rho}(\zeta) \leq \tau\right\}$. There exists a maximal $\tau_{0}$ for which $\sqrt{\rho}$ is well defined, known as the Grauert tube radius. For $\tau \leq \tau_{0}, M_{\tau}$ is a strictly pseudo-convex domain in $M_{\mathbb{C}}$.

The complexified exponential map $(x, \xi) \rightarrow \exp _{x} i \xi$ defines a diffeomorphism from $B_{\tau}^{*} M$ to $M_{\tau}$ and pulls back $\sqrt{\rho}$ to $|\xi|_{g}$. The one-complex dimensional null foliation of $\partial \bar{\partial} \sqrt{\rho}$, known as the 'Monge-Ampère' or Riemann foliation, are the complex curves $t+i \tau \rightarrow \tau \dot{\gamma}(t)$, where $\gamma$ is a geodesic, where $\tau>0$ and where $\tau \dot{\gamma}(t)$ denotes multiplication of the tangent vector to $\gamma$ by $\tau$. We refer to $[\mathbf{L S 1}, \mathbf{G L S}, \mathbf{Z} 8]$ for further discussion.

\subsection{Poisson operator and analytic Continuation of eigenfunc-} tions. The half-wave group of $(M, g)$ is the unitary group $U(t)=e^{i t \sqrt{\Delta}}$ generated by the square root of the positive Laplacian. Its Schwartz kernel is a distribution on $\mathbb{R} \times M \times M$ with the eigenfunction expansion

$$
U(t, x, y)=\sum_{j=0}^{\infty} e^{i t \lambda_{j}} \varphi_{j}(x) \varphi_{j}(y) .
$$

By the Poisson operator we mean the analytic continuation of $U(t)$ to positive imaginary time,

$$
e^{-\tau \sqrt{\Delta}}=U(i \tau)
$$

The eigenfunction expansion then converges absolutely to a real analytic function on $\mathbb{R}_{+} \times M \times M$.

Let $A(\tau)$ denote the operator of analytic continuation of a function on $M$ to the Grauert tube $M_{\tau}$. Since

$$
U_{\mathbb{C}}(i \tau) \varphi_{\lambda}=e^{-\tau \lambda} \varphi_{\lambda}^{\mathbb{C}},
$$

it is simple to see that

$$
A(\tau)=U_{\mathbb{C}}(i \tau) e^{\tau \sqrt{\Delta}}
$$


where $U_{\mathbb{C}}(i \tau, \zeta, y)$ is the analytic continuation of the Poisson kernel in $x$ to $M_{\tau}$. In terms of the eigenfunction expansion, one has

$$
U_{\mathbb{C}}(i \tau, \zeta, y)=\sum_{j=0}^{\infty} e^{-\tau \lambda_{j}} \varphi_{j}^{\mathbb{C}}(\zeta) \varphi_{j}(y), \quad(\zeta, y) \in M_{\epsilon} \times M
$$

This is a very useful observation because $U_{\mathbb{C}}(i \tau)(66)$ is a Fourier integral operator with complex phase and can be related to the geodesic flow. The analytic continuability of the Poisson operator to $M_{\tau}$ implies that every eigenfunction analytically continues to the same Grauert tube.

7.2. Analytic continuation of the Poisson wave group. The analytic continuation of the Poisson-wave kernel to $M_{\tau}$ in the $x$ variable is discussed in detail in $[\mathbf{Z 8}]$ and ultimately derives from the analysis by Hadamard of his parametrix construction. We only briefly discuss it here and refer to $[\mathbf{Z 8}]$ for further details. In the case of Euclidean $\mathbb{R}^{n}$ and its wave kernel $U(t, x, y)=\int_{\mathbb{R}^{n}} e^{i t|\xi|} e^{i\langle\xi, x-y\rangle} d \xi$ which analytically continues to $t+i \tau, \zeta=x+i p \in \mathbb{C}_{+} \times \mathbb{C}^{n}$ as the integral

$$
U_{\mathbb{C}}(t+i \tau, x+i p, y)=\int_{\mathbb{R}^{n}} e^{i(t+i \tau)|\xi|} e^{i\langle\xi, x+i p-y\rangle} d \xi
$$

The integral clearly converges absolutely for $|p|<\tau$.

Exact formulae of this kind exist for $S^{m}$ and $\mathbf{H}^{m}$. For a general real analytic Riemannian manifold, there exists an oscillatry integral expression for the wave kernel of the form,

$$
U(t, x, y)=\int_{T_{y}^{*} M} e^{i t|\xi|_{g}} e^{i\left\langle\xi, \exp _{y}^{-1}(x)\right\rangle} A(t, x, y, \xi) d \xi
$$

where $A(t, x, y, \xi)$ is a polyhomogeneous amplitude of order 0 . The holomorphic extension of (67) to the Grauert tube $|\zeta|<\tau$ in $x$ at time $t=i \tau$ then has the form

$$
U_{\mathbb{C}}(i \tau, \zeta, y)=\int_{T_{y}^{*}} e^{-\tau|\xi| g_{y}} e^{i\left\langle\xi, \exp _{y}^{-1}(\zeta)\right\rangle} A(t, \zeta, y, \xi) d \xi \quad(\zeta=x+i p) .
$$

7.3. Analytic continuation of eigenfunctions. A function $f \in$ $C^{\infty}(M)$ has a holomorphic extension to the closed tube $\sqrt{\rho}(\zeta) \leq \tau$ if and only if $f \in \operatorname{Dom}\left(e^{\tau \sqrt{\Delta}}\right)$, where $e^{\tau \sqrt{\Delta}}$ is the backwards 'heat operator' generated by $\sqrt{\Delta}$ (rather than $\Delta$ ). That is, $f=\sum_{n=0}^{\infty} a_{n} \varphi_{\lambda_{n}}$ admits an analytic continuation to the open Grauert tube $M_{\tau}$ if and only if $f$ is in the domain of $e^{\tau \sqrt{\Delta}}$, i.e. if $\sum_{n}\left|a_{n}\right|^{2} e^{2 \tau \lambda_{n}}<\infty$. Indeed, the analytic continuation is $U_{\mathbb{C}}(i \tau) e^{\tau \sqrt{\Delta}} f$. The subtlety is in the nature of the restriction to the boundary of the maximal Grauert tube.

This result generalizes one of the classical Paley-Wiener theorems to real analytic Riemannian manifolds [Bou, GS2]. In the simplest case of $M=S^{1}, f \sim \sum_{n \in \mathbb{Z}} a_{n} e^{i n \theta} \in C^{\omega}\left(S^{1}\right)$ is the restriction of a holomorphic function $F \sim \sum_{n \in \mathbb{Z}} a_{n} z^{n}$ on the annulus $S_{\tau}^{1}=\{|\log | z||<\tau\}$ and with 
$F \in L^{2}\left(\partial S_{\tau}^{1}\right)$ if and only if $\sum_{n}|\hat{f}(n)|^{2} e^{2|n| \tau}<\infty$. The case of $\mathbb{R}^{m}$ is more complicated since it is non-compact. We are mainly concerned with compact manifolds and so the complications are not very relevant here. But we recall that one of the classical Paley-Wiener theorems states that a real analytic function $f$ on $\mathbb{R}^{n}$ is the restriction of a holomorphic function on the closed tube $|\operatorname{Im} \zeta| \leq \tau$ which satisfies $\int_{\mathbb{R}^{m}}|F(x+i \xi)|^{2} d x \leq C$ for $\xi \leq \tau$ if and only if $\hat{f} e^{\tau|\operatorname{Im} \zeta|} \in L^{2}\left(\mathbb{R}^{n}\right)$.

Let us consider examples of holomorphic continuations of eigenfunctions:

- On the flat torus $\mathbb{R}^{m} / \mathbb{Z}^{m}$, the real eigenfunctions are $\cos \langle k, x\rangle$, $\sin \langle k, x\rangle$ with $k \in 2 \pi \mathbb{Z}^{m}$. The complexified torus is $\mathbb{C}^{m} / \mathbb{Z}^{m}$ and the complexified eigenfunctions are $\cos \langle k, \zeta\rangle, \sin \langle k, \zeta\rangle$ with $\zeta=x+i \xi$.

- On the unit sphere $S^{m}$, eigenfunctions are restrictions of homogeneous harmonic functions on $\mathbb{R}^{m+1}$. The latter extend holomorphically to holomorphic harmonic polynomials on $\mathbb{C}^{m+1}$ and restrict to holomorphic function on $S_{\mathbb{C}}^{m}$.

- On $\mathbf{H}^{m}$, one may use the hyperbolic plane waves $e^{(i \lambda+1)\langle z, b\rangle}$, where $\langle z, b\rangle$ is the (signed) hyperbolic distance of the horocycle passing through $z$ and $b$ to 0 . They may be holomorphically extended to the maximal tube of radius $\pi / 2$.

- On compact hyperbolic quotients $\mathbf{H}^{m} / \Gamma$, eigenfunctions can be then represented by Helgason's generalized Poisson integral formula $[\mathbf{H}]$,

$$
\varphi_{\lambda}(z)=\int_{B} e^{(i \lambda+1)\langle z, b\rangle} d T_{\lambda}(b) .
$$

Here, $z \in D$ (the unit disc), $B=\partial D$, and $d T_{\lambda} \in \mathcal{D}^{\prime}(B)$ is the boundary value of $\varphi_{\lambda}$, taken in a weak sense along circles centered at the origin 0 . To analytically continue $\varphi_{\lambda}$ it suffices to analytically continue $\langle z, b\rangle$. Writing the latter as $\langle\zeta, b\rangle$, we have:

$$
\varphi_{\lambda}^{\mathbb{C}}(\zeta)=\int_{B} e^{(i \lambda+1)\langle\zeta, b\rangle} d T_{\lambda}(b)
$$

7.4. Complexified spectral projections. The next step is to holomorphically extend the spectral projectors $d \Pi_{[0, \lambda]}(x, y)=\sum_{j} \delta(\lambda-$ $\left.\lambda_{j}\right) \varphi_{j}(x) \varphi_{j}(y)$ of $\sqrt{\Delta}$. The complexified diagonal spectral projections measure is defined by

$$
d_{\lambda} \Pi_{[0, \lambda]}^{\mathbb{C}}(\zeta, \bar{\zeta})=\sum_{j} \delta\left(\lambda-\lambda_{j}\right)\left|\varphi_{j}^{\mathbb{C}}(\zeta)\right|^{2}
$$

Henceforth, we generally omit the superscript and write the kernel as $\Pi_{[0, \lambda]}^{\mathbb{C}}(\zeta, \bar{\zeta})$. This kernel is not a tempered distribution due to the exponential growth of $\left|\varphi_{j}^{\mathbb{C}}(\zeta)\right|^{2}$. Since many asymptotic techniques assume spectral functions are of polynomial growth, we simultaneously consider the damped 
spectral projections measure

$$
d_{\lambda} P_{[0, \lambda]}^{\tau}(\zeta, \bar{\zeta})=\sum_{j} \delta\left(\lambda-\lambda_{j}\right) e^{-2 \tau \lambda_{j}}\left|\varphi_{j}^{\mathbb{C}}(\zeta)\right|^{2}
$$

which is a temperate distribution as long as $\sqrt{\rho}(\zeta) \leq \tau$. When we set $\tau=\sqrt{\rho}(\zeta)$ we omit the $\tau$ and put

$$
d_{\lambda} P_{[0, \lambda]}(\zeta, \bar{\zeta})=\sum_{j} \delta\left(\lambda-\lambda_{j}\right) e^{-2 \sqrt{\rho}(\zeta) \lambda_{j}}\left|\varphi_{j}^{\mathbb{C}}(\zeta)\right|^{2} .
$$

The integral of the spectral measure over an interval $I$ gives

$$
\Pi_{I}(x, y)=\sum_{j: \lambda_{j} \in I} \varphi_{j}(x) \varphi_{j}(y) .
$$

Its complexification gives the kernel (121) along the diagonal,

$$
\Pi_{I}(\zeta, \bar{\zeta})=\sum_{j: \lambda_{j} \in I}\left|\varphi_{j}^{\mathbb{C}}(\zeta)\right|^{2},
$$

and the integral of (71) gives its temperate version

$$
P_{I}^{\tau}(\zeta, \bar{\zeta})=\sum_{j: \lambda_{j} \in I} e^{-2 \tau \lambda_{j}}\left|\varphi_{j}^{\mathbb{C}}(\zeta)\right|^{2},
$$

or in the crucial case of $\tau=\sqrt{\rho}(\zeta)$,

$$
P_{I}(\zeta, \bar{\zeta})=\sum_{j: \lambda_{j} \in I} e^{-2 \sqrt{\rho}(\zeta) \lambda_{j}}\left|\varphi_{j}^{\mathbb{C}}(\zeta)\right|^{2}
$$

7.5. Poisson operator as a complex Fourier integral operator. The damped spectral projection measure $d_{\lambda} P_{[0, \lambda]}^{\tau}(\zeta, \bar{\zeta})(71)$ is dual under the real Fourier transform in the $t$ variable to the restriction

$$
U(t+2 i \tau, \zeta, \bar{\zeta})=\sum_{j} e^{(-2 \tau+i t) \lambda_{j}}\left|\varphi_{j}^{\mathbb{C}}(\zeta)\right|^{2}
$$

to the anti-diagonal of the mixed Poisson-wave group. The adjoint of the Poisson kernel $U(i \tau, x, y)$ also admits an anti-holomorphic extension in the $y$ variable. The sum (76) are the diagonal values of the complexified wave kernel

$$
\begin{aligned}
U\left(t+2 i \tau, \zeta, \bar{\zeta}^{\prime}\right)= & \int_{M} U(t+i \tau, \zeta, y) E\left(i \tau, y, \bar{\zeta}^{\prime}\right) d V_{g}(x) \\
& =\sum_{j} e^{(-2 \tau+i t) \lambda_{j}} \varphi_{j}^{\mathbb{C}}(\zeta) \overline{\varphi_{j}^{\mathbb{C}}\left(\zeta^{\prime}\right)} .
\end{aligned}
$$

We obtain (77) by orthogonality of the real eigenfunctions on $M$.

Since $U(t+2 i \tau, \zeta, y)$ takes its values in the CR holomorphic functions on $\partial M_{\tau}$, we consider the Sobolev spaces $\mathcal{O}^{s+\frac{n-1}{4}}\left(\partial M_{\tau}\right)$ of CR holomorphic functions on the boundaries of the strictly pseudo-convex domains $M_{\epsilon}$, i.e.

$$
\mathcal{O}^{s+\frac{m-1}{4}}\left(\partial M_{\tau}\right)=W^{s+\frac{m-1}{4}}\left(\partial M_{\tau}\right) \cap \mathcal{O}\left(\partial M_{\tau}\right),
$$


where $W_{s}$ is the sth Sobolev space and where $\mathcal{O}\left(\partial M_{\epsilon}\right)$ is the space of boundary values of holomorphic functions. The inner product on $\mathcal{O}^{0}\left(\partial M_{\tau}\right)$ is with respect to the Liouville measure

$$
d \mu_{\tau}=(i \partial \bar{\partial} \sqrt{\rho})^{m-1} \wedge d^{c} \sqrt{\rho} .
$$

We then regard $U(t+i \tau, \zeta, y)$ as the kernel of an operator from $L^{2}(M) \rightarrow$ $\mathcal{O}^{0}\left(\partial M_{\tau}\right)$. It equals its composition $\Pi_{\tau} \circ U(t+i \tau)$ with the Szegöprojector

$$
\Pi_{\tau}: L^{2}\left(\partial M_{\tau}\right) \rightarrow \mathcal{O}^{0}\left(\partial M_{\tau}\right)
$$

for the tube $M_{\tau}$, i.e. the orthogonal projection onto boundary values of holomorphic functions in the tube.

This is a useful expression for the complexified wave kernel, because $\tilde{\Pi}_{\tau}$ is a complex Fourier integral operator with a small wave front relation. More precisely, the real points of its canonical relation form the graph $\Delta_{\Sigma}$ of the identity map on the symplectic one $\Sigma_{\tau} \subset T^{*} \partial M_{\tau}$ spanned by the real one-form $d^{c} \rho$, i.e.

$$
\Sigma_{\tau}=\left\{\left(\zeta ; r d^{c} \rho(\zeta)\right), \quad \zeta \in \partial M_{\tau}, r>0\right\} \subset T^{*}\left(\partial M_{\tau}\right) .
$$

We note that for each $\tau$, there exists a symplectic equivalence $\Sigma_{\tau} \simeq T^{*} M$ by the map $\left(\zeta, r d^{c} \rho(\zeta)\right) \rightarrow\left(E_{\mathbb{C}}^{-1}(\zeta), r \alpha\right)$, where $\alpha=\xi \cdot d x$ is the action form (cf. [GS2]).

The following result was first stated by Boutet de Monvel (for more details, see also $[\mathbf{G S 2}, \mathbf{Z 8}])$.

TheOrem 7.1. [Bou, GS2] $\Pi_{\epsilon} \circ U(i \epsilon): L^{2}(M) \rightarrow \mathcal{O}\left(\partial M_{\epsilon}\right)$ is a complex Fourier integral operator of order $-\frac{m-1}{4}$ associated to the canonical relation

$$
\Gamma=\left\{\left(y, \eta, \iota_{\epsilon}(y, \eta)\right\} \subset T^{*} M \times \Sigma_{\epsilon} .\right.
$$

Moreover, for any s,

$$
\Pi_{\epsilon} \circ U(i \epsilon): W^{s}(M) \rightarrow \mathcal{O}^{s+\frac{m-1}{4}}\left(\partial M_{\epsilon}\right)
$$

is a continuous isomorphism.

In $[\mathbf{Z 8}]$ we give the following sharpening of the sup norm estimates of [Bou, GLS]:

Proposition 7.2. Suppose $(M, g)$ is real analytic. Then

$$
\sup _{\zeta \in M_{\tau}}\left|\varphi_{\lambda}^{\mathbb{C}}(\zeta)\right| \leq C \lambda^{\frac{m+1}{2}} e^{\tau \lambda}, \quad \sup _{\zeta \in M_{\tau}}\left|\frac{\partial \varphi_{\lambda}^{\mathbb{C}}(\zeta)}{\partial \zeta_{j}}\right| \leq C \lambda^{\frac{m+3}{2}} e^{\tau \lambda}
$$

The proof follows easily from the fact that the complexified Poisson kernel is a complex Fourier integral operator of finite order. The estimates can be improved further. 
7.6. Maximal plurisubharmonic functions and growth of $\varphi_{\lambda}^{\mathbb{C}}$. In $[\mathbf{Z 8}]$, we discussed analogues in the setting of Gruaert tubes for the basic notions of pluripotential theory on domains in $\mathbb{C}^{m}$. Of relevance here is that the Grauert tube function $\sqrt{\rho}$ is the analogue of the pluricomplex Green's function. We recall that the maximal PSH function (or pluri-complex Green's function) relative to a subset $E \subset \Omega$ is defined by

$$
V_{E}(\zeta)=\sup \left\{u(z): u \in P S H(\Omega),\left.u\right|_{E} \leq 0,\left.u\right|_{\partial \Omega} \leq 1\right\} .
$$

On a real analytic Riemannian manifold, the natural analogue of $\mathcal{P}^{N}$ is the space

$$
\mathcal{H}^{\lambda}=\left\{p=\sum_{j: \lambda_{j} \leq \lambda} a_{j} \varphi_{\lambda_{j}}, a_{1}, \ldots, a_{N(\lambda)} \in \mathbb{R}\right\}
$$

spanned by eigenfunctions with frequencies $\leq \lambda$. Rather than using the sup norm, it is convenient to work with $L^{2}$ based norms than sup norms, and so we define

$$
\mathcal{H}_{M}^{\lambda}=\left\{p=\sum_{j: \lambda_{j} \leq \lambda} a_{j} \varphi_{\lambda_{j}}, \quad\|p\|_{L^{2}(M)}^{2}=\sum_{j=1}^{N(\lambda)}\left|a_{j}\right|^{2}=1\right\} .
$$

We define the $\lambda$-Siciak extremal function by

$$
\Phi_{M}^{\lambda}(z)=\sup \left\{|\psi(z)|^{1 / \lambda}: \psi \in \mathcal{H}_{\lambda} ;\|\psi\|_{M} \leqslant 1\right\},
$$

and the extremal function by

$$
\Phi_{M}(z)=\sup _{\lambda} \Phi_{M}^{\lambda}(z) .
$$

The extremal PSH function is defined by

$$
V_{g}(\zeta ; \tau)=\sup \left\{u(z): u \in P S H\left(M_{\tau}\right),\left.u\right|_{M} \leq 0,\left.u\right|_{\partial M_{\tau}} \leq \tau\right\} .
$$

In $[\mathbf{Z 8}]$ we proved that $V_{g}=\sqrt{\rho}$ and that

$$
\Phi_{M}=V_{g} .
$$

The proof is based on the properties of (73). By using a Bernstein-Walsh inequality

$$
\frac{1}{N(\lambda)} \leq \frac{\Pi_{[0, \lambda]}(\zeta, \bar{\zeta})}{\Phi_{M}^{\lambda}(\zeta)^{2}} \leq C N(\lambda) e^{\epsilon N(\lambda)}
$$

it is not hard to show that

$$
\Phi_{M}(z)=\lim _{\lambda \rightarrow \infty} \frac{1}{\lambda} \log \Pi_{[0, \lambda}(\zeta, \bar{\zeta}) .
$$

To evaluate the logarithm, one can show that the kernel is essentially $e^{\lambda \sqrt{\rho}}$ times the temperate projection defined by the Poisson operator,

$$
P_{[0, \lambda]}(\zeta, \bar{\zeta})=\sum_{j: \lambda_{j} \in[0, \lambda]} e^{-2 \sqrt{\rho}(\zeta) \lambda_{j}}\left|\varphi_{j}^{\mathbb{C}}(\zeta)\right|^{2}
$$

The equality (80) follows from the fact that $\lim _{\lambda \rightarrow \infty} \frac{1}{\lambda} \log P_{[0, \lambda]}(\zeta, \bar{\zeta})=0$. 
We now return to nodal sets, where we will see the same extremal functions arise.

\section{Counting nodal lines which touch the boundary in analytic plane domains}

It is often possible to obtain more refined results on nodal sets by studying their intersections with some fixed (and often special) hypersurface. This has been most successful in dimension two. In this section, we review the results of $[\mathbf{T Z}]$ giving upper bounds on the number of intersections of the nodal set with the boundary of an analytic (or more generally piecewise analytic) plane domain. One may expect that the results of this section can also be generalized to higher dimensions by measuring codimension two nodal hypersurface volumes within the boundary.

Thus we would like to count the number of nodal lines (i.e. components of the nodal set) which touch the boundary. Here we assume that 0 is a regular value so that components of the nodal set are either loops in the interior (closed nodal loops) or curves which touch the boundary in two points (open nodal lines). It is known that for generic piecewise analytic plane domains, zero is a regular value of all the eigenfunctions $\varphi_{\lambda_{j}}$, i.e. $\nabla \varphi_{\lambda_{j}} \neq 0$ on $Z_{\varphi_{\lambda_{j}}}$ $[\mathbf{U}]$; we then call the nodal set regular. Since the boundary lies in the nodal set for Dirichlet boundary conditions, we remove it from the nodal set before counting components. Henceforth, the number of components of the nodal set in the Dirichlet case means the number of components of $Z_{\varphi_{\lambda_{j}}} \backslash \partial \Omega$.

In the following, and henceforth, $C_{\Omega}>0$ denotes a positive constant depending only on the domain $\Omega$.

THEOREM 8.1. Let $\Omega$ be a piecewise analytic domain and let $n_{\partial \Omega}\left(\lambda_{j}\right)$ be the number of components of the nodal set of the jth Neumann or Dirichlet eigenfunction which intersect $\partial \Omega$. Then there exists $C_{\Omega}$ such that $n_{\partial \Omega}\left(\lambda_{j}\right) \leq C_{\Omega} \lambda_{j}$.

By a piecewise analytic domain $\Omega^{2} \subset \mathbb{R}^{2}$, we mean a compact domain with piecewise analytic boundary, i.e. $\partial \Omega$ is a union of a finite number of piecewise analytic curves which intersect only at their common endpoints. Such domains are often studied as archtypes of domains with ergodic billiards and quantum chaotic eigenfunctions, in particular the Bunimovich stadium or Sinai billiard. Their nodal sets have been the subject of a number of numerical studies (e.g. [BGS, FGS]).

In general, there does not exist a non-trivial lower bound for the number of components touching the boundary. E.g. in a disc, the zero sets of the eigenfunctions are unions of circles concentric with the origin and spokes emanating from the center. Only the spokes intersect the boundary and their number reflects the angular momentum rather than the eigenvalue of the eigenfunction. But we conjecture that for piecewise analytic domains 
with ergodic billiards, the the number of complex zeros of $\left.\varphi_{\lambda_{j}}^{\mathbb{C}}\right|_{\partial \Omega_{\mathbb{C}}}$ is bounded below by $C_{\Omega} \lambda_{j}$. We discuss work in progress on this conjecture in $\S 10$.

In comparison to the order $O\left(\lambda_{j}\right)$ of the number of boundary nodal points, the total number of connected components of $Z_{\varphi_{\lambda_{j}}}$ has the upper bound $O\left(\lambda_{j}^{2}\right)$ by the Courant nodal domain theorem. It is not known in general whether the Courant upper bound is achieved, but we expect that it is often achieved in order of magnitude. In $[\mathbf{N S}]$ it is proved that the average number of nodal components of a random spherical harmonic is of order of magnitude $\lambda_{j}^{2}$. Thus, the number of components touching the boundary is one order of magnitude below the total number of components.

8.1. Boundary critical points. The article $[\mathbf{T Z}]$ also contains a similar estimate on the number of critical points of $\varphi_{\lambda_{j}}$ which occur on the boundary. We denote the boundary critical set by

$$
\mathcal{C}_{\varphi_{\lambda_{j}}}=\left\{q \in \partial \Omega:\left(d \varphi_{\lambda_{j}}\right)(q)=0\right\} .
$$

In the case of Neumann eigenfunctions, $q \in \mathcal{C}_{\varphi_{\lambda_{j}}} \Longleftrightarrow d\left(\left.\varphi_{\lambda_{j}}\right|_{\partial \Omega}(q)\right)=0$ since the normal derivative automatically equals zero on the boundary, while in the Dirichlet case $q \in \mathcal{C}_{\varphi_{\lambda_{j}}} \Longleftrightarrow \partial_{\nu} \varphi_{\lambda_{j}}(q)=0$ since the boundary is a level set.

We observe that radial eigenfunctions on the disc are constant on the boundary; thus, boundary critical point sets need not be isolated. We therefore impose a non-degeneracy condition on the tangential derivative $\partial_{t}\left(\left.\varphi_{\lambda_{j}}\right|_{\partial \Omega}\right)$ to ensure that its zeros are isolated and can be counted. We say that the Neumann problem for a bounded domain has the asymptotic Schiffer property if there exists $C>0$ such that, for all Neumann eigenfunctions $\varphi_{\lambda_{j}}$ with sufficiently large $\lambda_{j}$,

$$
\frac{\left\|\partial_{t} \varphi_{\lambda_{j}}\right\|_{L^{2}(\partial \Omega)}}{\left\|\varphi_{\lambda_{j}}\right\|_{L^{2}(\partial \Omega)}} \geq e^{-C \lambda_{j}} .
$$

Here, $\partial_{t}$ is the unit tangential derivative, and the $L^{2}$ norms refer to the restrictions of the eigenfunction to $\partial \Omega$.

TheORem 8.2. Let $\Omega \subset \mathbb{R}^{2}$ be piecewise real analytic. Suppose that $\left.\varphi_{\lambda_{j}}\right|_{\partial \Omega}$ satisfies the asymptotic Schiffer condition (83) in the Neumann case. Then the number of $n_{\text {crit }}\left(\lambda_{j}\right)=\# \mathcal{C}_{\varphi_{\lambda_{j}}}$ of critical points of a Neumann or Dirichlet eigenfunction $\varphi_{\lambda_{j}}$ which lie on $\partial \Omega$ satisfies $n_{\text {crit }}\left(\lambda_{j}\right) \leq C_{\Omega} \lambda_{j}$ for some $C_{\Omega}>0$

In the case of Dirichlet eigenfunctions, endpoints of open nodal lines are always boundary critical points, since they must be singular points of $\varphi_{\lambda_{j}}$. Hence, an upper bound for $n_{\text {crit }}\left(\lambda_{j}\right)$ also gives an upper bound for the number of open nodal lines.

Corollary 8.3. Suppose that $\Omega \subset \mathbb{R}^{2}$ is a piecewise real analytic plane domain. Let $n_{\partial \Omega}\left(\lambda_{j}\right)$ be the number of open nodal lines of the $j$ th Dirichlet 
eigenfunction, i.e. connected components of $\left\{\varphi_{\lambda_{j}}=0\right\} \subset \Omega^{o}$ whose closure intersects $\partial \Omega$. Then there exists $C_{\Omega}>0$ such that $n_{\partial \Omega}\left(\lambda_{j}\right) \leq C_{\Omega} \lambda_{j}$.

There does not exist a non-trivial lower bound on the number of interior critical points $[\mathbf{J N}]$.

8.2. Proof by analytic continuation. For the Neumann problem, the boundary nodal points are the same as the zeros of the boundary values $\left.\varphi_{\lambda_{j}}\right|_{\partial \Omega}$ of the eigenfunctions. The number of boundary nodal points is thus twice the number of open nodal lines. Hence in the Neumann case, Theorem 8.1 follows from:

THEOREM 8.4. Suppose that $\Omega \subset \mathbb{R}^{2}$ is a piecewise real analytic plane domain. Then the number $n\left(\lambda_{j}\right)=\# Z_{\varphi_{\lambda_{j}}} \cap \partial \Omega$ of zeros of the boundary values $\left.\varphi_{\lambda_{j}}\right|_{\partial \Omega}$ of the $j$ th Neumann eigenfunction satisfies $n\left(\lambda_{j}\right) \leq C_{\Omega} \lambda_{j}$, for some $C_{\Omega}>0$.

This is a more precise version of Theorem 8.1 since it does not assume that 0 is a regular value. In keeping with the theme of this survey, we prove Theorem 8.4 by analytically continuing the boundary values of the eigenfunctions and counting complex zeros and critical points of analytic continuations of Cauchy data of eigenfunctions. When $\partial \Omega \in C^{\omega}$, the eigenfunctions can be holomorphically continued to an open tube domain in $\mathbb{C}^{2}$ projecting over an open neighborhood $W$ in $\mathbb{R}^{2}$ of $\Omega$ which is independent of the eigenvalue. We denote by $\Omega_{\mathbb{C}} \subset \mathbb{C}^{2}$ the points $\zeta=x+i \xi \in \mathbb{C}^{2}$ with $x \in \Omega$. Then $\varphi_{\lambda_{j}}(x)$ extends to a holomorphic function $\varphi_{\lambda_{j}}^{\mathbb{C}}(\zeta)$ where $x \in W$ and where $|\xi| \leq \epsilon_{0}$ for some $\epsilon_{0}>0$.

Assuming $\partial \Omega$ real analytic, we define the (interior) complex nodal set by

$$
Z_{\varphi_{\lambda_{j}}}^{\mathbb{C}}=\left\{\zeta \in \Omega_{\mathbb{C}}: \varphi_{\lambda_{j}}^{\mathbb{C}}(\zeta)=0\right\}
$$

and the (interior) complex critical point set by

$$
\mathcal{C}_{\varphi_{\lambda_{j}}}^{\mathbb{C}}=\left\{\zeta \in \Omega_{\mathbb{C}}: d \varphi_{\lambda_{j}}^{\mathbb{C}}(\zeta)=0\right\} .
$$

THEOREM 8.5. Suppose that $\Omega \subset \mathbb{R}^{2}$ is a piecewise real analytic plane domain, and denote by $(\partial \Omega)_{\mathbb{C}}$ the union of the complexifications of its real analytic boundary components.

(1) Let $n\left(\lambda_{j}, \partial \Omega_{\mathbb{C}}\right)=\# Z_{\varphi_{\lambda_{j}}}^{\partial \Omega_{\mathbb{C}}}$ be the number of complex zeros on the complex boundary. Then there exists a constant $C_{\Omega}>0$ independent of the radius of $(\partial \Omega)_{\mathbb{C}}$ such that $n\left(\lambda_{j}, \partial \Omega_{\mathbb{C}}\right) \leq C_{\Omega} \lambda_{j}$.

(2) Suppose that the Neumann eigenfunctions satisfy (83) and let $n_{\text {crit }}\left(\lambda_{j}, \partial \Omega_{\mathbb{C}}\right)=\# \mathcal{C}_{\varphi_{\lambda_{j}}}^{\partial \Omega_{\mathbb{C}}}$. Then there exists $C_{\Omega}>0$ independent of the radius of $(\partial \Omega)_{\mathbb{C}}$ such that $n_{\text {crit }}\left(\lambda_{j}, \partial \Omega_{\mathbb{C}}\right) \leq C_{\Omega} \lambda_{j}$.

The theorems on real nodal lines and critical points follow from the fact that real zeros and critical points are also complex zeros and critical points, 
hence

$$
n\left(\lambda_{j}\right) \leq n\left(\lambda_{j}, \partial \Omega_{\mathbb{C}}\right) ; \quad n_{\text {crit }}\left(\lambda_{j}\right) \leq n_{\text {crit }}\left(\lambda_{j}, \partial \Omega_{\mathbb{C}}\right) .
$$

All of the results are sharp, and are already obtained for certain sequences of eigenfunctions on a disc (see $\S 4.6$ ). If the condition (83) is not satisfied, the boundary value of $\varphi_{\lambda_{j}}$ must equal a constant $C_{j}$ modulo an error of the form $o\left(e^{-C \lambda_{j}}\right)$. We conjecture that this forces the boundary values to be constant.

The method of proof of Theorem 8.5 generalizes from $\partial \Omega$ to a rather large class of real analytic curves $C \subset \Omega$, even when $\partial \Omega$ is not real analytic. Let us call a real analytic curve $C$ a good curve if there exists a constant $a>0$ so that for all $\lambda_{j}$ sufficiently large,

$$
\frac{\left\|\varphi_{\lambda_{j}}\right\|_{L^{2}(\partial \Omega)}}{\left\|\varphi_{\lambda_{j}}\right\|_{L^{2}(C)}} \leq e^{a \lambda_{j}}
$$

Here, the $L^{2}$ norms refer to the restrictions of the eigenfunction to $C$ and to $\partial \Omega$. The following result deals with the case where $C \subset \partial \Omega$ is an interior realanalytic curve. The real curve $C$ may then be holomorphically continued to a complex curve $C_{\mathbb{C}} \subset \mathbb{C}^{2}$ obtained by analytically continuing a real analytic parametrization of $C$.

Theorem 8.6. Suppose that $\Omega \subset \mathbb{R}^{2}$ is a $C^{\infty}$ plane domain, and let $C \subset \Omega$ be a good interior real analytic curve in the sense of (85). Let $n\left(\lambda_{j}, C\right)=\# Z_{\varphi_{\lambda_{j}}} \cap C$ be the number of intersection points of the nodal set of the $j$-th Neumann (or Dirichlet) eigenfunction with $C$. Then there exists $A_{C, \Omega}>0$ depending only on $C, \Omega$ such that $n\left(\lambda_{j}, C\right) \leq A_{C, \Omega} \lambda_{j}$.

A recent paper of J. Jung shows that many natural curves in the hyperbolic plane are 'good' $[\mathbf{J J}]$.

8.3. Application to Pleijel's conjecture. We also note an interesting application due to I. Polterovich [Po] of Theorem 8.1 to an old conjecture of A. Pleijel regarding Courant's nodal domain theorem, which says that the number $n_{k}$ of nodal domains (components of $\Omega \backslash Z_{\varphi_{\lambda_{k}}}$ ) of the $k$ th eigenfunction satisfies $n_{k} \leq k$. Pleijel $[\mathbf{P}]$ improved this result for Dirichlet eigefunctions of plane domains: For any plane domain with Dirichlet boundary conditions, $\lim \sup _{k \rightarrow \infty} \frac{n_{k}}{k} \leq \frac{4}{j_{1}^{2}} \simeq 0.691 \ldots$, where $j_{1}$ is the first zero of the $J_{0}$ Bessel function. He conjectured that the same result should be true for a free membrane, i.e. for Neumann boundary conditions. This was recently proved in the real analytic case by I. Polterovich $[\mathbf{P o}]$. His argument is roughly the following: Pleijel's original argument applies to all nodal domains which do not touch the boundary, since the eigenfunction is a Dirichlet eigenfunction in such a nodal domain. The argument does not apply to nodal domains which touch the boundary, but by Theorem 8.1 the number of such domains is negligible for the Pleijel bound. 


\section{Equidistribution of complex nodal sets of real ergodic eigenfunctions on analytic $(M, g)$ with ergodic geodesic flow}

We now consider global results when hypotheses are made on the dynamics of the geodesic flow. Use of the global wave operator brings into play the relation between the geodesic flow and the complexified eigenfunctions, and this allows one to prove gobal results on nodal hypersurfaces that reflect the dynamics of the geodesic flow. In some cases, one can determine not just the volume, but the limit distribution of complex nodal hypersurfaces. Since we have discussed this result elsewhere $[\mathbf{Z 6}]$ we only briefly review it here.

The complex nodal hypersurface of an eigenfunction is defined by

$$
Z_{\varphi_{\lambda}^{\mathbb{C}}}=\left\{\zeta \in B_{\epsilon_{0}}^{*} M: \varphi_{\lambda}^{\mathbb{C}}(\zeta)=0\right\} .
$$

There exists a natural current of integration over the nodal hypersurface in any ball bundle $B_{\epsilon}^{*} M$ with $\epsilon<\epsilon_{0}$, given by

$$
\left\langle\left[Z_{\varphi_{\lambda}^{\mathbb{C}}}\right], \varphi\right\rangle=\frac{i}{2 \pi} \int_{B_{\epsilon}^{*} M} \partial \bar{\partial} \log \left|\varphi_{\lambda}^{\mathbb{C}}\right|^{2} \wedge \varphi=\int_{Z_{\varphi_{\lambda}^{\mathbb{C}}}} \varphi, \quad \varphi \in \mathcal{D}^{(m-1, m-1)}\left(B_{\epsilon}^{*} M\right) .
$$

In the second equality we used the Poincaré-Lelong formula. The notation $\mathcal{D}^{(m-1, m-1)}\left(B_{\epsilon}^{*} M\right)$ stands for smooth test $(m-1, m-1)$-forms with support in $B_{\epsilon}^{*} M$.

The nodal hypersurface $Z_{\varphi_{\lambda}^{\mathbb{C}}}$ also carries a natural volume form $\left|Z_{\varphi_{\lambda}^{\mathbb{C}}}\right|$ as a complex hypersurface in a Kähler manifold. By Wirtinger's formula, it equals the restriction of $\frac{\omega_{g}^{m-1}}{(m-1) !}$ to $Z_{\varphi_{\lambda}^{\mathbb{C}}}$. Hence, one can regard $Z_{\varphi_{\lambda}^{\mathbb{C}}}$ as defining the measure

$$
\left\langle\left|Z_{\varphi_{\lambda}^{\mathbb{C}}}\right|, \varphi\right\rangle=\int_{Z_{\varphi_{\lambda}^{\mathbb{C}}}} \varphi \frac{\omega_{g}^{m-1}}{(m-1) !}, \quad \varphi \in C\left(B_{\epsilon}^{*} M\right) .
$$

We prefer to state results in terms of the current $\left[Z_{\varphi_{\lambda}^{\mathbb{C}}}\right]$ since it carries more information.

Theorem 9.1. Let $(M, g)$ be real analytic, and let $\left\{\varphi_{j_{k}}\right\}$ denote a quantum ergodic sequence of eigenfunctions of its Laplacian $\Delta$. Let $\left(B_{\epsilon_{0}}^{*} M, J\right)$ be the maximal Grauert tube around $M$ with complex structure $J_{g}$ adapted to g. Let $\epsilon<\epsilon_{0}$. Then:

$$
\frac{1}{\lambda_{j_{k}}}\left[Z_{\varphi_{j_{k}}^{\mathbb{C}}}\right] \rightarrow \frac{i}{\pi} \partial \bar{\partial} \sqrt{\rho} \text { weakly in } \mathcal{D}^{(1,1)}\left(B_{\epsilon}^{*} M\right)
$$

in the sense that, for any continuous test form $\psi \in \mathcal{D}^{(m-1, m-1)}\left(B_{\epsilon}^{*} M\right)$, we have

$$
\frac{1}{\lambda_{j_{k}}} \int_{Z_{\varphi_{j_{k}}^{\mathbb{C}}}} \psi \rightarrow \frac{i}{\pi} \int_{B_{\epsilon}^{*} M} \psi \wedge \partial \bar{\partial} \sqrt{\rho}
$$


Equivalently, for any $\varphi \in C\left(B_{\epsilon}^{*} M\right)$,

$$
\frac{1}{\lambda_{j_{k}}} \int_{Z_{\varphi_{j_{k}}^{\mathbb{C}}}} \varphi \frac{\omega_{g}^{m-1}}{(m-1) !} \rightarrow \frac{i}{\pi} \int_{B_{\epsilon}^{*} M} \varphi \partial \bar{\partial} \sqrt{\rho} \wedge \frac{\omega_{g}^{m-1}}{(m-1) !} .
$$

Corollary 9.2. Let $(M, g)$ be a real analytic with ergodic geodesic flow. Let $\left\{\varphi_{j_{k}}\right\}$ denote a full density ergodic sequence. Then for all $\epsilon<\epsilon_{0}$,

$$
\frac{1}{\lambda_{j_{k}}}\left[Z_{\varphi_{j_{k}}^{\mathbb{C}}}\right] \rightarrow \frac{i}{\pi} \partial \bar{\partial} \sqrt{\rho} \text {, weakly in } \mathcal{D}^{(1,1)}\left(B_{\epsilon}^{*} M\right) \text {. }
$$

The proof consists of three ingredients:

(1) By the Poincaré-Lelong formula, $\left[Z_{\varphi_{\lambda}^{\mathbb{C}}}\right]=i \partial \bar{\partial} \log \left|\varphi_{\lambda}^{\mathbb{C}}\right|$. This reduces the theorem to determining the limit of $\frac{1}{\lambda} \log \left|\varphi_{\lambda}^{\mathbb{C}}\right|$.

(2) $\frac{1}{\lambda} \log \left|\varphi_{\lambda}^{\mathbb{C}}\right|$ is a sequence of PSH functions which are uniformly bounded above by $\sqrt{\rho}$. By a standard compactness theorem, the sequence is pre-compact in $L^{1}$ : every sequence from the family has an $L^{1}$ convergent subsequence.

(3) $\left|\varphi_{\lambda}^{\mathbb{C}}\right|^{2}$, when properly $L^{2}$ normalized on each $\partial M_{\tau}$ is a quantum ergodic sequence on $\partial M_{\tau}$. This property implies that the $L^{2}$ norm of $\left|\varphi_{\lambda}\right|^{2}$ on $\partial M_{\tau}$ is asymptotically $e^{\lambda_{j} \tau}$.

(4) Ergodicity and the calculation of the $L^{2}$ norm imply that the only possible $L^{1}$ limit of $\frac{1}{\lambda} \log \left|\varphi_{\lambda}^{\mathbb{C}}\right|$ is $\sqrt{\rho}$. This concludes the proof.

We note that the first two steps are valid on any real analytic $(M, g)$. The difference is that the $L^{2}$ norms of $\varphi_{\lambda}^{\mathbb{C}}$ may depend on the subsequence and can often not equal $\sqrt{\rho}$. That is, $\frac{1}{\lambda}\left|\varphi_{\lambda}^{\mathbb{C}}\right|$ behaves like the maximal PSH function in the ergodic case, but not in general. For instance, on a flat torus, the complex zero sets of ladders of eigenfunctions concentrate on a real hypersurface in $M_{\mathbb{C}}$. This may be seen from the complexified real eigenfunctions $\sin \langle k, x+i \xi\rangle$, which vanish if and only if $\langle k, x\rangle \in 2 \pi \mathbb{Z}$ and $\langle k, \xi\rangle=0$. Here, $k \in \mathbb{N}^{m}$ is a lattice point. The exact limit distribution depends on which ray or ladder of lattice points one takes in the limit. The result reflects the quantum integrability of the flat torus, and a similar (but more complicated) description of the zeros exists in all quantum integrable cases. The fact that $\frac{1}{\lambda} \log \left|\varphi_{\lambda}^{\mathbb{C}}\right|$ is pre-compact on a Grauert tube of any real analytic Riemannian manifold confirms the upper bound on complex nodal hypersurface volumes.

\section{Intersections of nodal sets and gedoesics on real analytic surfaces}

In $\S 8$ we discussed upper bounds on the number of intersection points of the nodal set with the bounary of a real analytic plane domain and more general 'good' analytic curves. In this section, we discuss work in progress on intersections of nodal sets and geodesics on surfaces with ergodic geodesic flow. Of course, the results are only tentative but it seems worthwhile at 
this point in time to explain the role of ergodicity in obtaining lower bounds and asymptotics. We restrict to geodesic curves because they have rather special properties that makes the analysis somewhat different than for more general curves such as distance circles. The dimensional restriction is due to the fact that the results are partly based on the quantum ergodic restriction theorems of [TZ2, TZ3], which concern restrictions of eigenfunctions to hypersurfaces. Nodal sets and geodesics have complementary dimensions and intersect in points, and therefore it makes sense to count the number of intersections.

We fix $(x, \xi) \in S^{*} M$ and let

$$
\gamma_{x, \xi}: \mathbb{R} \rightarrow M, \quad \gamma_{x, \xi}(0)=x, \quad \gamma_{x, \xi}^{\prime}(0)=\xi \in T_{x} M
$$

denote the corresponding parametrized geodesic. Our goal is to determine the asymptotic distribution of intersection points of $\gamma_{x, \xi}$ with the nodal set of a highly eigenfunction. As usual, we cannot cope with this problem in the real domain and therefore analytically continue it to the complex domain. Thus, we consider the intersections

$$
\mathcal{N}_{\lambda_{j}}^{\gamma_{x, \xi}^{\mathbb{C}}}=Z_{\varphi_{j}^{\mathbb{C}}} \cap \gamma_{x, \xi}^{\mathbb{C}}
$$

of the complex nodal set with the (image of the) complexification of a generic geodesic If

$$
S_{\epsilon}=\{(t+i \tau \in \mathbb{C}:|\tau| \leq \epsilon\}
$$

then $\gamma_{x, \xi}$ admits an analytic continuation

$$
\gamma_{x, \xi}^{\mathbb{C}}: S_{\epsilon} \rightarrow M_{\epsilon}
$$

In other words, we consider the zeros of the pullback,

$$
\left\{\gamma_{x, \xi}^{*} \varphi_{\lambda}^{\mathbb{C}}=0\right\} \subset S_{\epsilon} .
$$

We encode the discrete set by the measure

$$
\left[\mathcal{N}_{\lambda_{j}}^{\gamma_{x, \xi}^{\mathbb{C}}}\right]=\sum_{(t+i \tau): \varphi_{j}^{\mathbb{C}}\left(\gamma_{x, \xi}^{\mathbb{C}}(t+i \tau)\right)=0} \delta_{t+i \tau} .
$$

We would like to show that for generic geodesics, the complex zeros on the complexified geodesic condense on the real points and become uniformly distributed with respect to arc-length. This does not always occur: as in our discussion of QER theorems, if $\gamma_{x, \xi}$ is the fixed point set of an isometric involution, then "odd" eigenfunctions under the involution will vanish on the geodesic. The additional hypothesis is that QER holds for $\gamma_{x, \xi}$, i.e. that Theorem 5.6 is valid. The following is proved $([\mathbf{Z 3}])$ :

THEOREM 10.1. Let $\left(M^{2}, g\right)$ be a real analytic Riemannian surface with ergodic geodesic flow. Let $\gamma_{x, \xi}$ satisfy the QER hypothesis. Then there exists 
a subsequence of eigenvalues $\lambda_{j_{k}}$ of density one such that for any $f \in C_{c}\left(S_{\epsilon}\right)$,

$$
\lim _{k \rightarrow \infty} \sum_{(t+i \tau): \varphi_{j}^{\mathbb{C}}\left(\gamma_{x, \xi}^{\mathbb{C}}(t+i \tau)\right)=0} f(t+i \tau)=\int_{\mathbb{R}} f(t) d t .
$$

In other words,

$$
\text { weak }^{*} \lim _{k \rightarrow \infty} \frac{i}{\pi \lambda_{j_{k}}}\left[\mathcal{N}_{\lambda_{j}}^{\gamma_{x, \xi}^{\mathbb{C}}}\right]=\delta_{\tau=0},
$$

in the sense of weak* convergence on $C_{c}\left(S_{\epsilon}\right)$. Thus, the complex nodal set intersects the (parametrized) complexified geodesic in a discrete set which is asymptotically (as $\lambda \rightarrow \infty$ ) concentrated along the real geodesic with respect to its arclength.

This concentration- equidistribution result is a 'restricted' version of the result of $\S 9$. As noted there, the limit distribution of complex nodal sets in the ergodic case is a singular current $d d^{c} \sqrt{\rho}$. The motivation for restricting to geodesics is that restriction magnifies the singularity of this current. In the case of a geodesic, the singularity is magnified to a delta-function; for other curves there is additionally a smooth background measure.

The assumption of ergodicity is crucial. For instance, in the case of a flat torus, say $\mathbb{R}^{2} / L$ where $L \subset \mathbb{R}^{2}$ is a generic lattice, the real eigenfunctions are $\cos \langle\lambda, x\rangle, \sin \langle\lambda, x\rangle$ where $\lambda \in L^{*}$, the dual lattice, with eigenvalue $-|\lambda|^{2}$. Consider a geodesic $\gamma_{x, \xi}(t)=x+t \xi$. Due to the flatness, the restriction $\sin \left\langle\lambda, x_{0}+t \xi_{0}\right\rangle$ of the eigenfunction to a geodesic is an eigenfunction of the Laplacian $-\frac{d^{2}}{d t^{2}}$ of submanifold metric along the geodesic with eigenvalue $-\left\langle\lambda, \xi_{0}\right\rangle^{2}$. The complexification of the restricted eigenfunction is $\sin \left\langle\lambda, x_{0}+\right.$ $\left.(t+i \tau) \xi_{0}\right\rangle \mid$ and its exponent of its growth is $\tau\left|\left\langle\frac{\lambda}{|\lambda|}, \xi_{0}\right\rangle\right|$, which can have a wide range of values as the eigenvalue moves along different rays in $L^{*}$. The limit current is $i \partial \bar{\partial}$ applied to the limit and thus also has many limits

The proof involves several new principles which played no role in the global result of $\S 9$ and which are specific to geodesics. However, the first steps in the proof are the same as in the global case. By the Poincaré-Lelong formula, we may express the current of summation over the intersection points in (92) in the form,

$$
\left[\mathcal{N}_{\lambda_{j}}^{\gamma_{x, \xi}^{\mathbb{C}}}\right]=i \partial \bar{\partial}_{t+i \tau} \log \left|\gamma_{x, \xi}^{*} \varphi_{\lambda_{j}}^{\mathbb{C}}(t+i \tau)\right|^{2}
$$

Thus, the main point of the proof is to determine the asymptotics of $\frac{1}{\lambda_{j}} \log \left|\gamma_{x, \xi}^{*} \varphi_{\lambda_{j}}^{\mathbb{C}}(t+i \tau)\right|^{2}$. When we freeze $\tau$ we put

$$
\gamma_{x, \xi}^{\tau}(t)=\gamma_{x, \xi}^{\mathbb{C}}(t+i \tau) .
$$

Proposition 10.2. (Growth saturation) If $\left\{\varphi_{j_{k}}\right\}$ satisfies QER along any arcs of $\gamma_{x, \xi}$, then in $L_{l o c}^{1}\left(S_{\tau}\right)$, we have

$$
\lim _{k \rightarrow \infty} \frac{1}{\lambda_{j_{k}}} \log \left|\gamma_{x, \xi}^{\tau *} \varphi_{\lambda_{j_{k}}}^{\mathbb{C}}(t+i \tau)\right|^{2}=|\tau| .
$$


Proposition 10.2 immediately implies Theorem 10.1 since we can apply $\partial \bar{\partial}$ to the $L^{1}$ convergent sequence $\frac{1}{\lambda_{j_{k}}} \log \left|\gamma_{x, \xi}^{*} \varphi_{\lambda_{j_{k}}}^{\mathbb{C}}(t+i \tau)\right|^{2}$ to obtain $\partial \bar{\partial}|\tau|$.

The upper bound in Proposition 10.2 follows immediately from the known global estimate

$$
\lim _{k \rightarrow \infty} \frac{1}{\lambda_{j}} \log \mid \varphi_{j_{k}}\left(\gamma_{x, \xi}^{\mathbb{C}}(\zeta)|\leq| \tau \mid\right.
$$

on all of $\partial M_{\tau}$. Hence the difficult point is to prove that this growth rate is actually obtained upon restriction to $\gamma_{x, \xi}^{\mathbb{C}}$. This requires new kinds of arguments related to the QER theorem.

- Complexifications of restrictions of eigenfunctions to geodesics have incommensurate Fourier modes, i.e. higher modes are exponentially larger than lower modes.

- The quantum ergodic restriction theorem in the real domain shows that the Fourier coefficients of the top allowed modes are 'large' (i.e. as large as the lower modes). Consequently, the $L^{2}$ norms of the complexified eigenfunctions along arcs of $\gamma_{x, \xi}^{\mathbb{C}}$ achieve the lower bound of Proposition 10.2.

- Invariance of Wigner measures along the geodesic flow implies that the Wigner measures of restrictions of complexified eigenfunctions to complexified geodesics should tend to constant multiples of Lebesgue measures $d t$ for each $\tau>0$. Hence the eigenfunctions everywhere on $\gamma_{x, \xi}^{\mathbb{C}}$ achieve the growth rate of the $L^{2}$ norms.

These principles are most easily understood in the case of periodic geodesics. We let $\gamma_{x, \xi}: S^{1} \rightarrow M$ parametrize the geodesic with arc-length (where $S^{1}=\mathbb{R} / L \mathbb{Z}$ where $L$ is the length of $\gamma_{x, \xi}$ ).

First, we use Theorem 5.6 to prove

Lemma 10.3. Assume that $\left\{\varphi_{j}\right\}$ satsifies QER along the periodic geodesic $\gamma_{x, \xi}$. Let $\left\|\gamma_{x, \xi}^{\tau *} \varphi_{j}^{\mathbb{C}}\right\|_{L^{2}\left(S^{1}\right)}^{2}$ be the $L^{2}$-norm of the complexified restriction of $\varphi_{j}$ along $\gamma_{x, \xi}^{\tau}$. Then,

$$
\lim _{\lambda_{j} \rightarrow \infty} \frac{1}{\lambda_{j}} \log \left\|\gamma_{x, \xi}^{\tau *} \varphi_{j}^{\mathbb{C}}\right\|_{L^{2}\left(S^{1}\right)}^{2}=|\tau| .
$$

To prove Lemma 10.3, we study the orbital Fourier series of $\gamma_{x, \xi}^{\tau *} \varphi_{j}$ and of its complexification. The orbital Fourier coefficients are

$$
\nu_{\lambda_{j}}^{x, \xi}(n)=\frac{1}{L_{\gamma}} \int_{0}^{L_{\gamma}} \varphi_{\lambda_{j}}\left(\gamma_{x, \xi}(t)\right) e^{-\frac{2 \pi i n t}{L_{\gamma}}} d t
$$

and the orbital Fourier series is

$$
\varphi_{\lambda_{j}}\left(\gamma_{x, \xi}(t)\right)=\sum_{n \in \mathbb{Z}} \nu_{\lambda_{j}}^{x, \xi}(n) e^{\frac{2 \pi i n t}{L \gamma}}
$$


Hence the analytic continuation of $\gamma_{x, \xi}^{\tau *} \varphi_{j}$ is given by

$$
\varphi_{\lambda_{j}}^{\mathbb{C}}\left(\gamma_{x, \xi}(t+i \tau)\right)=\sum_{n \in \mathbb{Z}} \nu_{\lambda_{j}}^{x, \xi}(n) e^{\frac{2 \pi i n(t+i \tau)}{L \gamma}} .
$$

By the Paley-Wiener theorem for Fourier series, the series converges absolutely and uniformly for $|\tau| \leq \epsilon_{0}$. By "energy localization" only the modes with $|n| \leq \lambda_{j}$ contribute substantially to the $L^{2}$ norm. We then observe that the Fourier modes decouple, since they have different exponential growth rates. We use the QER hypothesis in the following way:

Lemma 10.4. Suppose that $\left\{\varphi_{\lambda_{j}}\right\}$ is QER along the periodic geodesic $\gamma_{x, \xi}$. Then for all $\epsilon>0$, there exists $C_{\epsilon}>0$ so that

$$
\sum_{n:|n| \geq(1-\epsilon) \lambda_{j}}\left|\nu_{\lambda_{j}}^{x, \xi}(n)\right|^{2} \geq C_{\epsilon} .
$$

Lemma 10.4 implies Lemma 10.3 since it implies that for any $\epsilon>0$,

$$
\sum_{n:|n| \geq(1-\epsilon) \lambda_{j}}\left|\nu_{\lambda_{j}}^{x, \xi}(n)\right|^{2} e^{-2 n \tau} \geq C_{\epsilon} e^{2 \tau(1-\epsilon) \lambda_{j}} .
$$

To go from asymptotics of $L^{2}$ norms of restrictions to Proposition 10.2 we then use the third principle:

Proposition 10.5. (Lebesgue limits) If $\gamma_{x, \xi}^{*} \varphi_{j} \neq 0$ (identically), then for all $\tau>0$ the sequence

$$
U_{j}^{x, \xi, \tau}=\frac{\gamma_{x, \xi}^{\tau *} \varphi_{j}^{\mathbb{C}}}{\left\|\gamma_{x, \xi}^{\tau *} \varphi_{j}^{\mathbb{C}}\right\|_{L^{2}\left(S^{1}\right)}}
$$

is QUE with limit measure given by normalized Lebesgue measure on $S^{1}$.

The proof of Proposition 10.2 is completed by combining Lemma 10.3 and Proposition 10.5. Theorem 10.1 follows easily from Proposition 6.1.

The proof for non-periodic geodesics is considerably more involved, since one cannot use Fourier analysis in quite the same way.

\section{Nodal and critical sets of Riemannian random waves}

We mentioned above that Riemannian random waves provide a probabilistic model that is conjectured to predict the behavior of eigenfunctions when the geodesic flow of $(M, g)$ is ergodic. In this section, we define the model precisely as in $[\mathbf{Z 4}]$ (see also [Nic] for a similar model) and survey some of the current results and conjectures. We should emphasize that some of the rigorous results on zeros or critical points of Riemannian random

waves, both in the real and complex domain, are much simpler than for individual eigenfuntions, and therefore do not provide much guidance on how to prove results for an orthonormal basis of eigenfunctions. But the relative 
simplicity of random waves and their value as predictors provide the motivation for studying random waves. And there are many hopelessly difficult problems on random waves as well, which we will survey in this section.

For expository simplicity we assume that the geodesic flow $G^{t}$ of $(M, g)$ is of one of the following two types:

(1) aperiodic: The Liouville measure of the closed orbits of $G^{t}$, i.e. the set of vectors lying on closed geodesics, is zero; or

(2) periodic $=$ Zoll: $G^{T}=i d$ for some $T>0$; henceforth $T$ denotes the minimal period. The common Morse index of the $T$-periodic geodesics will be denoted by $\beta$.

In the real analytic case, $(M, g)$ is automatically one of these two types, since a positive measure of closed geodesics implies that all geodesics are closed. The two-term Weyl laws counting eigenvalues of $\sqrt{\Delta}$ are very different in these two cases.

(1) In the aperiodic case, Ivrii's two term Weyl law states

$$
N(\lambda)=\#\left\{j: \lambda_{j} \leq \lambda\right\}=c_{m} \operatorname{Vol}(M, g) \lambda^{m}+o\left(\lambda^{m-1}\right)
$$

where $m=\operatorname{dim} M$ and where $c_{m}$ is a universal constant.

(2) In the periodic case, the spectrum of $\sqrt{\Delta}$ is a union of eigenvalue clusters $C_{N}$ of the form

$$
C_{N}=\left\{\left(\frac{2 \pi}{T}\right)\left(N+\frac{\beta}{4}\right)+\mu_{N i}, i=1 \ldots d_{N}\right\}
$$

with $\mu_{N i}=0\left(N^{-1}\right)$. The number $d_{N}$ of eigenvalues in $C_{N}$ is a polynomial of degree $m-1$.

We refer to [HoI-IV, Z4] for background and further discussion.

To define Riemannian random waves, we partition the spectrum of $\sqrt{\Delta_{g}}$ into certain intervals $I_{N}$ of width one and denote by $\Pi_{I_{N}}$ the spectral projections for $\sqrt{\Delta_{g}}$ corresponding to the interval $I_{N}$. The choice of the intervals $I_{N}$ is rather arbitrary for aperiodic $(M, g)$ and as mentioned above we assume $I_{N}=[N, N+1]$. In the Zoll case, we center the intervals around the center points $\frac{2 \pi}{T} N+\frac{\beta}{4}$ of the $N$ th cluster $C_{N}$. We call call such a choice of intervals a cluster decomposition. We denote by $d_{N}$ the number of eigenvalues in $I_{N}$ and put $\mathcal{H}_{N}=\operatorname{ran} \Pi_{I_{N}}$ (the range of $\Pi_{I_{N}}$ ).

We choose an orthonormal basis $\left\{\varphi_{N j}\right\}_{j=1}^{d_{N}}$ for $\mathcal{H}_{N}$. For instance, on $S^{2}$ one can choose the real and imaginary parts of the standard $Y_{m}^{N}$ 's. We endow the real vector space $\mathcal{H}_{N}$ with the Gaussian probability measure $\gamma_{N}$ defined by

$$
\gamma_{N}(f)=\left(\frac{d_{N}}{\pi}\right)^{d_{N} / 2} e^{-d_{N}|c|^{2}} d c, \quad f=\sum_{j=1}^{d_{\lambda}} c_{j} \varphi_{N j}, \quad d_{N}=\operatorname{dim} \mathcal{H}_{N}
$$

Here, $d c$ is $d_{N^{-}}$-dimensional real Lebesgue measure. The normalization is chosen so that $\mathbb{E}_{\gamma_{N}}\langle f, f\rangle=1$, where $\mathbb{E}_{\gamma_{N}}$ is the expected value with respect to $\gamma_{N}$. Equivalently, the $d_{N}$ real variables $c_{j}\left(j=1, \ldots, d_{N}\right)$ are independent 
identically distributed (i.i.d.) random variables with mean 0 and variance $\frac{1}{2 d_{N}}$; i.e.,

$$
\mathbb{E}_{\gamma_{N}} c_{j}=0, \quad \mathbb{E}_{\gamma_{N}} c_{j} c_{k}=\frac{1}{2 d_{N}} \delta_{j k}
$$

We note that the Gaussian ensemble is equivalent to picking $f_{N} \in \mathcal{H}_{N}$ at random from the unit sphere in $\mathcal{H}_{N}$ with respect to the $L^{2}$ inner product.

Depending on the choice of intervals, we obtain the following special ensembles:

- The asymptotically fixed frequency ensemble $\mathcal{H}_{I_{\lambda}}$, where $I_{\lambda}=$ $[\lambda, \lambda+1]$ and where $\mathcal{H}_{I_{\lambda}}$ is the vector space of linear combinations

$$
f_{\lambda}=\sum_{j: \lambda_{j} \in[\lambda, \lambda+1]} c_{j} \varphi_{\lambda_{j}}
$$

of eigenfunctions with $\lambda_{j}$ (the frequency) in an interval $[\lambda, \lambda+1]$ of fixed width. (Note that it is the square root of the eigenvalue of $\Delta$, not the eigenvalue, which is asymptotically fixed).

- The high frequency cut-off ensembles $\mathcal{H}_{[0, \lambda]}$ where the frequency is cut-off at $\lambda$ :

$$
f_{\lambda}=\sum_{j: \lambda_{j} \leq \lambda} c_{j} \varphi_{\lambda_{j}}
$$

- The cut-off Gaussian free field,

$$
f_{\lambda}=\sum_{j: \lambda_{j} \leq \lambda} c_{j} \frac{\varphi_{\lambda_{j}}}{\lambda_{j}} .
$$

One could use more general weights $w\left(\lambda_{j}\right)$ on a Sobolev space of functions or distributions on $M$. In the physics terminology, $w\left(\lambda_{j}\right)$ (or it square) is referred to as the power spectrum.

The key reason why we can study the limit distribution of nodal sets in this ensemble is that the covariance kernel

$$
\Pi_{I_{N}}(x, y)=\mathbb{E}_{\gamma_{N}}\left(f_{N}(x) f_{N}(y)\right)=\sum_{j: \lambda_{j} \in I_{N}} \varphi_{\lambda_{j}}(x) \varphi_{\lambda_{j}}(y)
$$

is the spectral projections kernel for $\sqrt{\Delta}$.

11.1. Equidistribution of nodal sets for almost all sequences of random waves. The real zeros are straightforward to define. For each $f_{\lambda} \in \mathcal{H}_{[0, \lambda]}$ or $\mathcal{H}_{I_{\lambda}}$ we associated to the zero set $Z_{f_{\lambda}}=\left\{x \in M: f_{\lambda}(x)=0\right\}$ the positive measure

$$
\left\langle\left|Z_{f_{\lambda}}\right|, \psi\right\rangle=\int_{Z_{f_{\lambda}}} \psi d \mathcal{H}^{n-1},
$$

where $d \mathcal{H}^{m-1}$ is the induced (Hausdorff) hypersurface measure. 
The main result we review is the limit law for random sequences of random real Riemannian waves. By a random sequence, we mean an element of the product probability space

$$
\mathcal{H}_{\infty}=\prod_{N=1}^{\infty} \mathcal{H}_{N}, \quad \gamma_{\infty}=\prod_{N=1}^{N} \gamma_{N}
$$

THEOREM 11.1. [ZZ] Let $(M, g)$ be a compact Riemannian manifold, and let $\left\{f_{N}\right\}$ be a random sequence in (103). Then

$$
\frac{1}{N} \sum_{n=1}^{N} \frac{1}{\lambda_{n}}\left|Z_{f_{n}}\right| \rightarrow d V_{g} \quad \text { almost surely w.r.t. }\left(\mathcal{H}_{\infty}, \gamma_{\infty}\right) \text {. }
$$

11.2. Mean and variance. We first show that the normalized expected limit distribution $\frac{1}{\lambda} \mathbb{E}\left|Z_{f_{\lambda}}\right|$ of zeros of random Riemannian waves tends to the volume form $d V_{g}$ as $\lambda \rightarrow \infty$. That is, we define the 'linear statistic',

$$
X_{\psi}^{N}\left(f_{N}\right)=\left\langle\psi,\left|Z_{f_{N}}\right|\right\rangle, \quad \psi \in C(M)
$$

and then define

$$
\left\langle\mathbb{E}_{\gamma_{N}}\left|Z_{f_{N}}\right|, \psi\right\rangle=\mathbb{E}_{\gamma_{N}} X_{\psi}^{N},
$$

Theorem 11.2. Let $(M, g)$ be a compact Riemannian manifold,let $\mathcal{H}_{[0, \lambda]}$ be the cutoff ensemble and let $\left(\mathcal{H}_{N}, \gamma_{N}\right)$ be the ensemble of Riemannian waves of asymptotically fixed frequency. Then in either ensemble:

(1) For any $C^{\infty}(M, g), \lim _{N \rightarrow \infty} \frac{1}{N} \mathbf{E}_{\gamma_{N}}\left\langle\left|Z_{f_{N}}\right|, \psi\right\rangle=\int_{M} \psi d V_{g}$.

(2) For a real analytic $\left.(M, g), \operatorname{Var}\left(\frac{1}{N} X_{\psi}^{N}\right)\right) \leq C$.

We restrict to real analytic metrics in (2) for the sake of brevity. In that case, the variance estimate follows easily from Theorem 2.1.

11.3. Density of real zeros. The formula for the density of zeros of random elements of $\mathcal{H}_{N}$ can be derived from the general Kac-Rice formula [BSZ1, BSZ2, Nic]:

$$
\mathbb{E}\left|Z_{f_{N}}\right|=K_{1}^{N}(z) d V_{g}, \quad K_{1}^{N}(x)=\int D(0, \xi, x)\|\xi\| d \xi .
$$

Here, $D(q, \xi, x) d q d \xi$ is the joint probability distribution of the Gaussian random variables $(\psi(x), \nabla \psi(x))$, i.e. the pushforward of the Gaussian measure on $\left.\mathcal{H}_{\lambda}\right)$ under the map $\psi \rightarrow(\psi(x), \nabla \psi(x))$. Note that the factor $\operatorname{det}\left(\xi \xi^{*}\right)$ in $[\mathbf{B S Z 1}, \mathbf{B S Z 2}]$ equals $\|\xi\|^{2}$ in the codimension one case. Indeed, let $d f_{x}^{*}$ be the adjoint map with respect to the inner product $g$ on $T_{x} M$. Let $d f_{x} \circ d f_{x}^{*}: \mathbb{R} \rightarrow \mathbb{R}$ be the composition. By $\operatorname{det} d f_{x} \circ d f_{x}^{*}$ is meant the determinant with respect to the inner product on $T_{x} M$; it clearly equals $|d f|^{2}$ in the codimension one case. 
The formulae of [BSZ1, BSZ2] (the 'Kac-Rice' formulae) give that

$$
D(0, \xi ; z)=Z_{n}(z) D_{\Lambda}(\xi ; z),
$$

where

$$
D_{\Lambda}(\xi ; z)=\frac{1}{\pi^{m} \sqrt{\operatorname{det} \Lambda}} \exp \left(-\left\langle\Lambda^{-1} \xi, \xi\right\rangle\right)
$$

is the Gaussian density with covariance matrix

$$
\Lambda=C-B^{*} A^{-1} B=\left(C_{q^{\prime}}^{q}-B_{q} A^{-1} B_{q^{\prime}}\right), \quad(q=1, \ldots, m)
$$

and

$$
Z(x)=\frac{\sqrt{\operatorname{det} \Lambda}}{\pi \sqrt{\operatorname{det} \Delta}}=\frac{1}{\pi \sqrt{A}} .
$$

Here,

$$
\begin{aligned}
& \Delta=\Delta^{N}(x)=\left(\begin{array}{cc}
A^{N} & B^{N} \\
B^{N *} & C^{N}
\end{array}\right), \\
&\left(A^{N}\right)=\mathbb{E}\left(X^{2}\right)=\frac{1}{d_{N}} \Pi_{I_{N}}(x, x), \\
&\left(B^{N}\right)_{q}= \mathbb{E}\left(X \Xi_{q}\right)=\left.\frac{1}{d_{N}} \frac{\partial}{\partial y_{q}} \Pi_{I_{N}}(x, y)\right|_{x=y}, \\
&\left(C^{\lambda}\right)_{q^{\prime}}^{q}= \mathbb{E}\left(\Xi_{q} \Xi_{q^{\prime}}\right)=\left.\frac{1}{d_{N}} \frac{\partial^{2}}{\partial x_{q} \partial y_{q^{\prime}}} \Pi_{I_{N}}(x, y)\right|_{x=y}, \\
& q, q^{\prime}=1, \ldots, m .
\end{aligned}
$$

Making a simple change of variables in the integral (106), we have

Proposition 11.3. [BSZ1] On a real Riemannian manifold of dimension $m$, the density of zeros of a random Riemannian wave is

$$
K_{1}^{N}(x)=\frac{1}{\pi^{m}\left(\sqrt{d_{N}^{-1} \Pi_{I_{N}}(x, x)}\right.} \int_{\mathbb{R}^{m}}\left\|\Lambda^{N}(x)^{1 / 2} \xi\right\| \exp (-\langle\xi, \xi\rangle) d \xi,
$$

where $\Lambda^{N}(x)$ is a symmetric form on $T_{x} M$. For the asymptotically fixed freqency ensembles, it is given by

$$
\begin{aligned}
\Lambda^{N}(x)=\frac{1}{d_{N}}( & \left.d_{x} \otimes d_{y} \Pi_{I_{N}}(x, y)\right|_{x=y} \\
& \left.\quad-\left.\left.\frac{1}{\Pi_{I_{N}}(x, y)} d_{x} \Pi_{I_{N}}(x, y)\right|_{x=y} \otimes d_{y} \Pi_{I_{N}}(x, y)\right|_{x=y}\right) .
\end{aligned}
$$

In the cutoff ensemble the formula is the same except that $\Pi_{I_{N}}$ is replaced by $\Pi_{[0, N]}$.

We then need the asymptotics of the matrix elements of $\Delta^{N}(x)$. They are simplest for the round sphere, so we state them first in that case: 
Proposition 11.4. Let $\Pi_{N}: L^{2}\left(S^{m}\right) \rightarrow \mathcal{H}_{N}$ be the orthogonal projection. Then:

- (A) $\Pi_{N}(x, x)=\frac{1}{\operatorname{Vol}\left(S^{m}\right)} d_{N}$;

- (B) $\left.d_{x} \Pi_{N}(x, y)\right|_{x=y}=\left.d_{y} \Pi_{N}(x, y)\right|_{x=y}=0$;

- $\left.(C) d_{x} \otimes d_{y} \Pi_{N}(x, y)\right|_{x=y}=\frac{1}{m V o l\left(S^{m}\right)} \lambda_{N}^{2} d_{N} g_{x}$.

We refer to $[\mathbf{Z 4}]$ for the calculation, which is quite simple because of the invariance under rotations. The expected density of random nodal hypersurfaces is given as follows

Proposition 11.5. In the case of $S^{m}$,

$$
K_{1}^{N}(x)=C_{m} \lambda_{N} \sim C_{m} N,
$$

where $C_{m}=\frac{1}{\pi^{m}} \int_{\mathbb{R}^{m}}|\xi| \exp (-\langle\xi, \xi\rangle) d \xi$.

Proof. By Propositiosn 11.3 and 11.4, we have

$$
K_{1}^{N}(x)=\frac{\sqrt{\operatorname{Vol}\left(S^{m}\right)}}{\pi^{m}} \int_{\mathbb{R}^{m}}\left\|\Lambda^{N}(x)^{1 / 2} \xi\right\| \exp (-\langle\xi, \xi\rangle) d \xi,
$$

where

$$
\Lambda^{N}(x)=\frac{1}{d_{N}}\left(\frac{1}{m \operatorname{Vol}\left(S^{m}\right)} \lambda_{N}^{2} d_{N} g_{x}\right) .
$$

11.4. Random Riemannian waves: proof of Theorem 11.2. We now generalize the result to any compact $C^{\infty}$ Riemannian manifold $(M, g)$ which is either aperiodic or Zoll. As in the case of $S^{m}$, the key issue is the asymptotic behavior of derivatives of the spectral projections

$$
\Pi_{I_{N}}(x, y)=\sum_{j: \lambda_{j} \in I_{N}} \varphi_{\lambda_{j}}(x) \varphi_{\lambda_{j}}(y) .
$$

Proposition 11.6. Assume $(M, g)$ is either aperiodic and $I_{N}=[N, N+$ 1] or Zoll and $I_{N}$ is a cluster decomposition. Let $\Pi_{I_{N}}: L^{2}(M) \rightarrow \mathcal{H}_{N}$ be the orthogonal projection. Then:

- (A) $\Pi_{I_{N}}(x, x)=\frac{1}{\operatorname{Vol}(M, g))} d_{N}(1+o(1))$;

- (B) $\left.d_{x} \Pi_{I_{N}}(x, y)\right|_{x=y}=\left.d_{y} \Pi_{N}(x, y)\right|_{x=y}=o\left(N^{m}\right)$;

- $\left.(C) d_{x} \otimes d_{y} \Pi_{I_{N}}(x, y)\right|_{x=y}=\frac{1}{\operatorname{Vol}(M, g))} \lambda_{N}^{2} d_{N} g_{x}(1+o(1))$.

In the aperiodic case,

(1) $\Pi_{[0, \lambda]}(x, x)=C_{m} \lambda^{m}+o\left(\lambda^{m-1}\right)$;

(2) $\left.d_{x} \otimes d_{y} \Pi_{[0, \lambda]}(x, y)\right|_{x=y}=C_{m} \lambda^{m+2} g_{x}+o\left(\lambda^{m+1}\right)$.

In the Zoll case, one adds the complete asymptotic expansions for $\Pi_{I_{N}}$ over the $N$ clusters to obtain expansions for $\Pi_{N}$.

We then have: 
Proposition 11.7. For the asymptotically fixed frequency ensemble, and for any $C^{\infty}(M, g)$ which is either Zoll or aperiodic (and with $I_{N}$ as in Proposition 11.6), we have

$$
\begin{aligned}
K_{1}^{N}(x) & =\frac{1}{\pi^{m}\left(\lambda_{N}\right)^{m / 2}} \int_{\mathbb{R}^{m}}\|\xi\| \exp \left(-\frac{1}{\lambda_{N}}\langle\xi, \xi\rangle\right) d \xi+o(1) \\
& \sim C_{m} N
\end{aligned}
$$

where $C_{m}=\frac{1}{\pi^{m}} \int_{\mathbb{R}^{m}}\|\xi\| \exp (-\langle\xi, \xi\rangle) d \xi$. The same formula holds for the cutoff ensemble.

Proof. Both on a sphere $S^{m}$ or on a more general $(M, g)$ which is either Zoll or aperiodic, we have by Propositions 11.4 resp. 11.6 and the general formula for $\Delta^{N}$ in $\S 11.3$ that

$$
\Delta^{N}(z)=\frac{1}{\operatorname{Vol}(M, g)}\left(\begin{array}{cc}
(1+o(1)) & o(1) \\
o(1) & N^{2} g_{x}(1+o(1))
\end{array}\right)
$$

It follows that

$$
\Lambda^{N}=C^{N}-B^{N *}\left(A^{N}\right)^{-1} B^{N}=\frac{1}{\operatorname{Vol}(M, g)} N^{2} g_{x}+o(N)
$$

Thus, we have

$$
\begin{aligned}
K_{1}^{N}(x) & \sim \frac{\sqrt{V o l(M, g)}}{\pi^{m}} \int_{\mathbb{R}^{m}}\left\|\Lambda^{N}(x)^{1 / 2} \xi\right\| \exp (-\langle\xi, \xi\rangle) d \xi \\
& =\frac{N}{\pi^{m}} \int_{\mathbb{R}^{m}}\left\|(I+o(1))(x)^{1 / 2} \xi\right\| \exp (-\langle\xi, \xi\rangle) d \xi
\end{aligned}
$$

where $o(1)$ denotes a matrix whose norm is $o(1)$, as as $N \rightarrow \infty$ we obtain the stated asymptotics.

So far, we have only determined the expected values of the nodal hypersurface measures. To complete the proof of Theorem 11.2, we need to prove:

Proposition 11.8. If $(M, g)$ is real analytic, then the variance of $\frac{1}{\lambda_{N}} X_{\psi}^{N}$ is bounded.

Proof. By Theorem 2.1, for $f_{N} \in \mathcal{H}_{I_{N}}, \frac{1}{\lambda_{N}} Z_{f_{N}}$ has bounded mass. Hence, the random variable $\frac{1}{\lambda_{N}} X_{\psi}^{N}$ is bounded, and therefore so is its variance. 
Remark: The variance of $\frac{1}{\lambda_{N}} X_{\psi}^{N}$ is given by

$$
\begin{aligned}
& \operatorname{Var}\left(\frac{1}{\lambda_{N}} X_{\psi}^{N}\right) \\
& \quad=\frac{1}{\lambda_{N}^{2}} \int_{M} \int_{M}\left(K_{2}^{N}(x, y)-K_{1}^{N}(x) K_{1}^{N}(y)\right) \psi(x) \psi(y) d V_{g}(x) d V_{g}(y),
\end{aligned}
$$

where $K_{2}^{N}(x, y)=\mathbb{E}_{\gamma_{N}}\left(Z_{f_{N}}(x) \otimes Z_{f_{N}}(y)\right)$ is the pair correlation function for zeros. Hence, boundedness would follow from

$$
\frac{1}{\lambda_{N}^{2}} \int_{M} \int_{M} K_{2}^{N}(x, y) d V_{g}(x) d V_{g}(y) \leq C .
$$

There is a formula similar to that for the density in Proposition 11.3 for $K_{2}^{N}(x, y)$ and it is likely that it could be used to prove boundedness of the variance for any $C^{\infty}$ Riemannian manifold.

11.5. Random sequences and proof of Theorem 11.1. We recall that the set of random sequences of Riemannian waves of increasing frequency is the probability space $\mathcal{H}_{\infty}=\prod_{N=1}^{\infty} \mathcal{H}_{I_{N}}$ with the measure $\gamma_{\infty}=\prod_{N=1}^{\infty} \gamma_{N}$. An element in $\mathcal{H}_{\infty}$ will be denoted $\mathbf{f}=\left\{f_{N}\right\}$. We have,

$$
\left|\left(\frac{1}{\lambda_{N}} Z_{f_{N}}, \psi\right)\right| \leq \frac{1}{\lambda_{N}} \mathcal{H}^{n-1}\left(Z_{f_{N}}\right)\|\psi\|_{C^{0}} .
$$

By a density argument it suffices to prove that the linear statistics $\frac{1}{\lambda_{N}}\left(Z_{f_{N}}, \psi\right)-\frac{1}{\operatorname{Vol}(M, g)} \int_{M} \psi d V_{g} \rightarrow 0$ almost surely in $\mathcal{H}_{\infty}$ We know that

(i) $\lim _{N \rightarrow \infty} \frac{1}{N} \sum_{k \leq N} \mathbb{E}\left(\frac{1}{\lambda_{k}} X_{\psi}^{k}\right)=\frac{1}{\operatorname{Vol}(M, g)} \int_{M} \psi d V_{g}$;

(ii) $\operatorname{Var}\left(\frac{1}{\lambda_{N}} X_{\psi}^{N}\right)$ is bounded on $\mathcal{H}_{\infty}$.

Since $\frac{1}{\lambda_{N}} X_{\psi}^{N}$ for $\{, N=1,2, \ldots\}$ is a sequence of independent random variables in $\mathcal{H}_{\infty}$ with bounded variances, the Kolmogorov strong law of large numbers implies that

$$
\lim _{N \rightarrow \infty} \frac{1}{N} \sum_{k \leq N}\left(\frac{1}{\lambda_{k}} X_{\psi}^{k}\right)=\frac{1}{\operatorname{Vol}(M, g)} \int_{M} \psi d V_{g}
$$

almost surely.

11.6. Complex zeros of random waves. We now state a complex analogue of the equidistribution of real nodal sets and show that it agrees with the the limit formula of Theorem 9.1.

We complexify Riemannian random waves as

$$
f_{N}^{\mathbb{C}}=\sum_{j=1}^{d_{N}} c_{N j} \varphi_{N j}^{\mathbb{C}}
$$

We note that the coefficients $c_{N j}$ are real and that the Gaussian measure on the coefficients remains the real Gaussian measure $\gamma_{N}$. The two point 
function is the analytic extensions to the totally real anti-diagonal in $M_{\mathbb{C}} \times$ $M_{\mathbb{C}}$ is therefore

$$
\mathbb{E}\left(\left|f_{N}(\zeta)\right|^{2}\right)=\Pi_{I_{N}}(\zeta, \bar{\zeta})=\sum_{j: \lambda_{j} \in I_{k}}\left|\varphi_{j}^{\mathbb{C}}(\zeta)\right|^{2}
$$

As in the proof of Theorem 9.1, the current of integration over the complex zero set

$$
Z_{f_{N}^{\mathbb{C}}}=\left\{\zeta \in M_{\mathbb{C}}: f_{N}^{\mathbb{C}}=0\right\}
$$

is the $(1,1)$ current defined by

$$
\left\langle\left[Z_{f_{N}^{\mathbb{C}}}\right], \psi\right\rangle=\int_{Z_{f_{N}^{\mathbb{C}}}} \psi, \quad \psi \in \mathcal{D}^{m-1, m-1}\left(M_{\mathbb{C}}\right),
$$

for smooth test forms of bi-degree $(m-1, m-1)$. In terms of scalar functions $\psi$ we may define $Z_{f_{N}^{\mathbb{C}}}$ as the measure,

$$
\left\langle\left[Z_{f_{N}^{\mathbb{C}}}\right], \psi\right\rangle=\int_{Z_{f_{N}^{\mathbb{C}}}} \psi \omega_{g}^{m-1} /(m-1) !,
$$

where $\omega_{g}=i \partial \bar{\partial} \rho$ is the Kählermetric adapted to $g$.

The proof of the next result is close to the proof of Theorem 9.1 and we therefore refer to $[\mathbf{Z 4}]$ for the details:

THEOREM 11.9. [Z4] Let $(M, g)$ be a real analytic compact Riemannian manifold. Then for either of the Riemannian random wave ensembles

$$
\mathbb{E}_{\gamma_{N}}\left(\frac{1}{N}\left[Z_{f_{N}^{\mathbb{C}}}\right]\right) \rightarrow \frac{i}{\pi} \partial \bar{\partial}|\xi|_{g}, \text { weakly in } \mathcal{D}^{\prime}(1,1)\left(B_{\epsilon}^{*} M\right) \text {. }
$$

As mentioned above, this result shows that the complex zeros of the random waves have the same expected limit distribution found in [Z3] for real analytic compact Riemannian manifolds with ergodic geodesic flow.

\section{Percolation heuristics}

In this final section, we review some of the more speculative conjectures relating nodal sets of both eigenfunctions and random waves to percolation theory. The conjectures are often quoted and it therefore seems worthwhile to try to state them precisely. The only rigorous result to date regarding eigenfunctions is the theorem of Nazarov-Sodin on the expected number of nodal domains for random spherical harmonics [NS] (see [Z5] for a brief over-view).

The percolation conjectures concern the statistics of sizes of nodal domains or nodal components. They are based on the idea that the nodal domains resemble percolation clusters. One might measure the 'size' of a nodal component $A_{\lambda_{j}}$ by its hypersurface area $\mathcal{H}^{n-1}\left(A_{\lambda, j}\right)$, and a nodal domain $D_{\lambda, j}$ by its volume $\mathcal{H}^{n}\left(D_{\lambda, j}\right)$. Let us restrict to the case of surfaces. 
For the purposes of this article, we introduce the term length spectrum of the nodal set as the set

$$
\operatorname{Lsp}\left(\varphi_{\lambda}\right)=\left\{\left(\mathcal{H}^{1}\left(C_{\lambda, j}\right): Z_{\varphi_{\lambda}}=\bigcup C_{\lambda ; j}\right\}\right.
$$

of lengths of its components, counted with multiplicity. It is encoded by the empirical measure of surface areas

$$
d \mu_{L}=\frac{1}{\mathcal{H}^{1}\left(Z_{\varphi_{\lambda}}\right)} \sum_{C_{\lambda, j}} \delta_{\mathcal{H}^{1}\left(C_{\lambda, j}\right)} \in \mathcal{P}_{1}(\mathbb{R}),
$$

(where $\mathcal{P}(\Omega)$ is the set of probability measures on $\Omega$ ), or equivalently by the length distribution function,

$$
\mathcal{L}_{\lambda}(t)=\sum_{j: \mathcal{H}^{1}\left(C_{\lambda, j}\right) \leq t} \mathcal{H}^{1}\left(C_{\lambda, j}\right) .
$$

We also consider the area spectrum,

$$
\operatorname{Asp}\left(\varphi_{\lambda}\right)=\left\{\left(\mathcal{H}^{2}\left(A_{\lambda, j}\right): M \backslash Z_{\varphi_{\lambda}}=\bigcup A_{\lambda ; j}\right\},\right.
$$

encoded by its empirical measure It is encoded by the empirical measure of surface areas

$$
d \mu_{A}=\frac{1}{\operatorname{Area}(M)} \sum_{A_{\lambda, j}} \delta_{\mathcal{H}^{2}\left(A_{\lambda, j}\right)} \in \mathcal{P}_{1}(\mathbb{R}),
$$

or by the area distribution function,

$$
\mathcal{A}_{\lambda}(t)=\sum_{j: \mathcal{H}^{2}\left(A_{\lambda, j}\right) \leq t} \mathcal{H}^{2}\left(A_{\lambda, j}\right) .
$$

Of course, there are some obvious constraints on such spectra; e.g. in the analytic case, there could only exist $O(\lambda)$ components with $\mathcal{H}^{1}$-length of order 1 , and only a bounded number of order $\lambda$.

In computer graphics of eigenfunctions on plane domains or surfaces, one sees many 'small' components $C_{\lambda, j}$ of the nodal set whose length appears to be of order $\frac{1}{\lambda}$. But one also sees long snaky nodal lines. How long are they? Do they persist as $\lambda \rightarrow \infty$ ? Roughly speaking, one may ask what proportion of the components come in sizes with different orders of magnitude. Of course, this depends on how many components there are, so it could be simpler to work with $\mathcal{L}\left(\varphi_{\lambda}\right), \mathcal{A}\left(\varphi_{\lambda}\right)$.

- How many components have $\mathcal{H}^{n-1}$-surface measure which is $\geq C \lambda^{\gamma}$ for some given $0<\gamma \leq 1$. It is possible that some individual nodal component has $\mathcal{H}^{n-1}$-surface area commensurate with that of the entire nodal set, as in the Lewy spherical harmonics with just two or three nodal components [Lew].

- How many components have $\mathcal{H}^{n-1}$-surface measure (i.e. length in dimension two) which is bounded below by a constant $C>0$ independent of $\lambda$ ? Such components are sometimes termed "percolating 
nodal lines" since their hypersurface volume is commensurate with the size of the macroscopic object (i.e. $M$ ).

- How many components have $\mathcal{H}^{n-1}$-surface measure of the minimal order $\frac{1}{\lambda}$ ?

The percolation conjectures relate the asymptotic distribution of lengths of nodal components and areas of nodal domains of eigenfunctions as defined in (123)-(126) to lengths of boundaries and areas of percolation clusters at criticality. There are different types of conjectures for the fixed frequency ensemble and the high frequency cutoff ensemble (see $\S 11$ for the definitions). According to the random wave hypothesis, the conjectures concerning the fixed frequency ensemble (e.g. random spherical harmonics of fixed degree) should also apply to nodal sets of eigenfunctions of quantum chaotic systems.

Percolation theory is concerned with connectivity and transport in a complex system. In particular, it studies connected clusters of objects in a random graph. In bond percolation the edges of the graph are independently open or closed with some probability $p$. The open edges form a subgraph whose connected components form the clusters. In site percolation the vertices are open or closed and an open path is a path through open vertices. The open cluster $C(v)$ of a vertex is the set of all open vertices which are connected to $v$ by an open path.

There also exists an analogous continuum percolation theory for level sets of random functions. We will assume the random functions are Gaussian Riemannian random waves on a surface. The main problem is to study the connectivity properties of level sets $\{f=t\}$. One imagines a random landscape of lakes and islands depending on the variable height $t$ of the water, the islands being the super-level sets $\{f>t\}$ of the random functions. For high water levels, the islands are disconnected, but as the water level is lowered the islands become more connected. At a critical level $t_{c}$ they 'percolate', i.e. it is possible to traverse the landscape while remaining on the land. A review with many illustrations is given by Isichenko [Isi] (see Section E (c), pages 980-984). As explained in [Isi] page 984, the contour lines of a random potential are associated to hulls of percolation clusters. Hence the area spectrum (125) is similar to the set of sizes of connected clusters in a percolation model.

In the physics literature, the random functions are usually functions on $\mathbb{R}^{2}$ (or possibly higher dimensional $\mathbb{R}^{n}$ ) and the Gaussian measure on the space of functions corresponds to a Hilbert space inner product. The Hilbert space is usually taken to be a Sobolev space, so that the inner product has the form $\int w(\xi)|\hat{f}(\xi)|^{2} d \xi$ (where $\hat{f}$ is the Fourier transform of $f$ ) and $w(\xi)=|\xi|^{2(1+\zeta)}$. The case $\zeta=0$ is known as the Gaussian free field (or massless scalar field) and is quite special in two dimensions since then the inner product $\int_{\mathbb{R}^{2}}|\nabla f|^{2} d x$ is conformally invariant. There are rigorous results on level sets of discretizations of the Gaussian free field and their 
continuum limits in $[\mathbf{S S}, \mathbf{M i}]$, with authoritative comments on the physics literature.

For purposes of this exposition, we assume the Riemannian random waves fall are of the types discussed in $\S 11$. In all cases, we truncate the frequency above a spectral parameter $\lambda$ and consider asymptotics as $\lambda \rightarrow \infty$. In this high frequency limit, the random waves oscillate more rapidly on the length scale $\frac{1}{\lambda}$. Since the conjectures and results depend strongly on the chosen weight $w$, we break up the discussion into two cases as in $\S 11$ : the high frequency cutoff ensemble and the fixed frequency ensemble. For each ensemble we let $\mathbb{E}_{\lambda}$ denote the expectation with respect to the Gaussian measure on the relevant space of linear combinations. Then we may ask for the asymptotic behavior of the expected distribution of lengths of nodal lines, resp. area of nodal domains

$$
\mathbb{E}_{\lambda} d \mu_{L}, \quad \mathbb{E}_{\lambda} d \mu_{A},
$$

where $d \mu_{L}$, resp. $d \mu_{A}$ are the empirical measures of lengths (123) of nodal lines, resp. areas (126) of nodal domains.

12.1. High frequency cutoff ensembles. The distribution of contour lengths of certain Gaussian random surfaces over $\mathbb{R}^{2}$ was studied at the physics level of rigor in $[\mathbf{K H}]$. They define the Gaussian measure as $e^{-f_{\zeta}(h)} d h$ where the 'free energy' is defined by

$$
f_{\zeta}(h)=\frac{K}{2} \int_{\mathbb{R}^{2}} \chi\left(\frac{|\xi|}{\lambda}\right)|\hat{h}(\xi)|^{2}|\xi|^{2(1+\zeta)} d \xi,
$$

where $\chi$ is a cutoff function to $[0,1]$ (they use the notation $a$ for $\frac{1}{\lambda}$ in our notation). When $\zeta=0$, this is a truncated Gaussian free field (truncated at frequencies $\leq \lambda)$ and its analogue on a surface $(M, g)$ is the Riemannian random wave model with spectral interval $[0, \lambda]$ and weight $w(\lambda)=\frac{1}{\lambda}$. The parameter $\zeta$ is referred to as the 'roughness exponent' in the physics literature. In the case of the Gaussian free field $\zeta=0$ the inner product is the Dirichlet inner product $\int_{\mathbb{R}^{2}}|\nabla f|^{2} d x$.

An important feature of the ensembles is scale-invariance. In the special case $\zeta=0$ (and dimension two), the Dirichlet inner product $\int_{M}|\nabla f|_{g}^{2} d A_{g}$ is conformally invariant, i.e. invariant under conformal changes $g \rightarrow e^{u} g$ of the Riemannian metric. When $\zeta \neq 0$ this is not the case, but it is assumed in $[\mathbf{K H}]$ that the fluctuations of the random Gaussian surface with height function $h$ are invariant under the rescaling $h(r) \rightarrow c^{-\zeta} h(c r)$ for any $c>1$. The authors of $[\mathbf{K H}]$ then make a number of conjectures concerning the distribution of contour lengths, which we interpret as conjectures concerning $\mathbb{E} d \mu_{L}$. First, they consider contours (i.e. level sets) through a fixed point $x_{0}$ and measure its length with the re-scaled arc-length measure $\lambda d s$, i.e. with arclength $s$ in units of $\frac{1}{\lambda}$. They define the fractal dimension of a nodal line component as the dimension $D$ so that $s \sim R^{D}$ where $R$ is the radius of the nodal component (i.e. half the diameter). They define $P(s)$ as the probability density that the contour through $x_{0}$ has length $s$. The principal claim is that 
$P(s) \sim s^{-\tau-1}$ satisfies a power law for some exponent $\tau([\mathbf{K H}](4))$. They also defines the distribution of loop lengths $P(s) \sim P(s) / s$ as the probability density that a random component has length $s$. We interpret their $\tilde{P(s)}$ as the density of $\lim _{\lambda \rightarrow \infty} \mathbb{E} d \mu_{L}$ with respect to $d s$ on $\mathbb{R}$. We thus interpret their conjecture as saying that a unique weak* limit of this family of measures exists and has a density relative to $d s$ with a power law decay as above.

The claims are based in part on scaling properties of the contour ensemble. They also are based in part on the expectation that, at 'criticality', the key percolation 'exponents' of power laws are universal and therefore should be the same for the discrete and continuum percolation theories (see e.g. $[\mathbf{I s i K}])$. In $[\mathbf{K H}]$, the authors suggest that when a certain roughness exponent $\zeta$ vanishes (the critical models), the continuum problem is related to the four-state Potts model. The $q$-state Potts model is an Ising type spin model on a lattice where each spin can take one of $q$ values. It is known to be related to connectivity and percolation problems on a graph $[\mathbf{B a x}, \mathbf{W u}]$.

They compute $D, \tau$ by relating both to another exponent $x_{1}$ defined by a "contour correlation function" $\mathcal{G}_{1}(r)$, which measures the probability that points at $x, x+r$ lie on the same contour loop. They claim that $\mathcal{G}_{1}(r) \sim|r|^{-2 x_{1}}$. They claim that $D(3-\tau)=2-2 x_{1}$ and $D(\tau-1)=2-\zeta$. As a result, $D=2-x_{1}-\zeta / 2, \tau-1=\frac{2-\zeta}{2-x_{1}-\zeta / 2}$. From the mapping to the four-state Potts model, they conclude that $x_{1}=\frac{1}{2}$.

There exist rigorous results in $[\mathbf{S S}, \mathbf{M i}]$ relating discretizations of the Gaussian free field (rather than high frequency truncations) to the percolation models. They prove that in various senses, the zero set of the discrete Gaussian free field tends to an $S L E_{4}$ curve. It does not seem to be known at present if zero sets of the high frequency truncation of the Gaussian free field also tends in the same sense to an $S L E_{4}$ curve. Note that the SLE curves are interfaces and that one must select one component of the zero set that should tend to an SLE curve. There might exist modified conjectures regarding CLE curves.

To determine the 'critical exponents' in continuum percolation, it is tempting to find a way to 'map' the continuum problem to a discrete percolation model. A geometric 'map' from a random wave to a graph is to associate to the random function its Morse-Smale decomposition, known in the physics literature as the "Morse skeleton" (see $\S 2.6$ or [Web] for an extensive exposition). As discussed in [Wei], and as illustrated in Figure 10 of [Isi], the Morse complex of the random function plays the role of the lattice in lattice percolation theory.

12.2. Fixed frequency ensembles. We now consider Riemannian random waves of asymtotically fixed frequency $\lambda$, such as random spherical harmonics of fixed degree or Euclidean random plane waves of fixed eigenvalue. In this case the weight is a delta function at the frequency. One would expect different behavior in the level sets since only one frequency is involved rather than the superposition of waves of all frequencies $\leq \lambda$. 
A recent exposition in the specific setting of random Euclidean eigenfunctions of fixed frequency is given by [EGJS]. The level sets play the role of open paths. Super-level sets are compared to clusters of sites in a critical 2D percolation model, such as bond percolation on a lattice. Each site may of the percolation model may be visualized as a disc of area $\frac{2 \pi^{2}}{\lambda^{2}}$, i.e. as a small component. The nodal domains may be thought of as connected clusters of a number $n$ such discs. Since nodal domains are connected components in which the eigenfunction is either positive + or negative - , they are analogous to clusters of 'open' or 'closed' vertices.

The main conjectures in this fixed frequency ensemble are due to E. Bogolmony and C. Schmidt $[\mathbf{B S}]$. They conjecture that the continuum percolation problem should belong to the same universality class as the Potts model at a certain critical point (where $q$ is related to a certain temperature) for a large rectangular lattice and that the nodal lines in the $\lambda \rightarrow \infty$ limit tend to $S L E_{6}$ curves. This is similar to the predictions of $[\mathbf{K H}]$ but for a very different ensemble where there is little apriori reason to expect conformal invariance in the limit. There are parallel conjectures in $[\mathbf{B B C F}]$ for zero-vorticity isolines in $2 \mathrm{D}$ turbulence, which are also conjectured to tend to $S L E_{6}$ curves. They remark (page 127) that this limit is surprising since continuous percolation models assume short-correlations in the height functions whereas the vorticity field correlations decay only like $r^{-4 / 3}$. They write, "When the pair correlation function falls off slower than $r^{-3 / 2}$, the system is not expected generally to belong to the universality class of uncorrelated percolation and to be conformally invariant". The same remarks apply to the fixed frequency ensemble, where the correlation function is the spectral projection $\Pi_{[\lambda, \lambda+1]}(x, y)$ for a fixed frequency. In this case, the correlations decay quite slowly as $r^{-\frac{1}{2}}$; we refer to [BS2] for this background and also for an argument why the nodal sets should nevertheless resemble conformally invariant $S L E_{g}$ curves.

If the nodal lines in the fixed frequency model are equivalent to the critical percolation model, then the 'probability' of finding a nodal domain of area $s$ should decay like $s^{-\tau}$ where $\tau=\frac{187}{91}>2$ (see [SA], p. 52 for the percolation theory result). Under some shape assumptions adopted in [EGJS], it is equivalent that the probability of finding clusters consisting of $n$ discs is of order $n^{-\tau}$. For random spherical harmonics, one may ask for the probability that a spherical harmonic of degree $N$ has size $n$. For a fixed $(M, g)$ with simple eigenvalues, this notion of probability from percolation theory does not make sense, but we might assume that the number of of nodal components is of order $\lambda^{2}$ and ask what proportion of the nodal components has size 1 . To obtain a percolating nodal line, one would need a cluster with $n=\lambda$ sites, and thus the proportion of such nodal components to the total number would be of order $\lambda^{-\tau}$. Thus, if there are $C \lambda^{2}$ total components, the number of such components would be around $\lambda^{2-\tau}=\lambda^{-\frac{5}{91}}<1$, so the model seems to predict that such macroscopic 
nodal lines are quite rare. It also predicts that the 'vast majority' of nodal components are close to the minimal size, which does not seem so evident from the computer graphics.

\section{References}

[AP] J. C. Alvarez Paiva and E. Fernandes, Gelfand transforms and Crofton formulas. Selecta Math. (N.S.) 13 (2007), no. 3, 369-390.

[AP2] J. C. Alvarez Paiva and G. Berck, What is wrong with the Hausdorff measure in Finsler spaces. Adv. Math. 204 (2006), no. 2, 647-663.

[Ar] S. Ariturk, Lower bounds for nodal sets of Dirichlet and Neumann eigenfunctions, to appear in Comm. Math. Phys. ( arXiv:1110.6885).

[Ba] L. Bakri, Critical set of eigenfunctions of the Laplacian, arXiv:1008.1699.

[Bae] C. Bär, On nodal sets for Dirac and Laplace operators. Comm. Math. Phys. 188 (1997), no. 3, 709-721.

[Bax] R. J. Baxter, Potts model at the critical temperature, Journal of Physics C: Solid State Physics 6 (1973), L445.

[BBCF] D. Bernard, G. Boffetta, A. Celani, and G. Falkovich, Conformal invariance in two-dimensional turbulence, nature. physics Vol. 2 (2002), p. 134.

[Ber] M. V. Berry, Regular and irregular semiclassical wavefunctions. J. Phys. A 10 (1977), no. 12, 2083-2091.

[Bers] L. Bers, Local behavior of solutions of general linear elliptic equations. Comm. Pure Appl. Math. 8 (1955), 473-496.

[BGS] G. Blum, S. Gnutzmann and U. Smilansky, Nodal domain statistics: A Criterion for quantum chaos, Phys. Rev. Lett. 88, 114101 (2002).

[BDS] E. Bogomolny, R. Dubertrand, and C. Schmit, SLE description of the nodal lines of random wavefunctions. J. Phys. A 40 (2007), no. 3, 381-395.

[BS] E. Bogomolny and C. Schmit, Percolation model for nodal domains of chaotic wave functions, Phys. Rev. Letters 88 (18) (2002), 114102-114102-4.

[BS2] E. Bogomolny and C. Schmit, Random wavefunctions and percolation. J. Phys. A 40 (2007), no. 47, 14033-14043.

[Br] J. Brüning, Über Knoten von Eigenfunktionen des Laplace-Beltrami Operators", Math. Z. 158 (1978), 15-21.

[BSZ1] P. Bleher, B Shiffman, and S. Zelditch, Universality and scaling of zeros on symplectic manifolds. Random matrix models and their applications, 31-69, Math. Sci. Res. Inst. Publ., 40, Cambridge Univ. Press, Cambridge, 2001.

[BSZ2] P. Bleher, B. Shiffman and S. Zelditch, Universality and scaling of correlations between zeros on complex manifolds, Invent. Math. 142 (2000), no. 2, 351-395. http://xxx.lanl.gov/abs/math-ph/9904020.

[Bourg] J. Bourgain, Geodesic restrictions and $L^{p}$-estimates for eigenfunctions of Riemannian surfaces, Linear and complex analysis, 27-35, Amer. Math. Soc. Tranl. Ser. 2, 226, Amer. Math. Soc., Providence, RI, 2009.

[BZ] J. Bourgain and Z. Rudnick, On the nodal sets of toral eigenfunctions. Invent. Math. 185 (2011), no. 1, 199-23.

[Bou] L. Boutet de Monvel, Convergence dans le domaine complexe des séries de fonctions propres. C. R. Acad. Sci. Paris Sér. A-B 287 (1978), no. 13, A855A856.

[BGT] N. Burq, P. Gérard, and N. Tzvetkov, Restrictions of the Laplace-Beltrami eigenfunctions to submanifolds. Duke Math. J. 138 (2007), no. 3, 445-486

[Bu] N. Burq, Quantum ergodicity of boundary values of eigenfunctions: A control theory approach, to appear in Canadian Math. Bull. (math.AP/0301349). 
[Ch1] S. Y. Cheng, Eigenfunctions and eigenvalues of Laplacian. Differential geometry (Proc. Sympos. Pure Math., Vol. XXVII, Stanford Univ., Stanford, Calif., 1973), Part 2, pp. 185-193. Amer. Math. Soc., Providence, R.I., 1975.

[Ch2] S. Y. Cheng, Eigenfunctions and nodal sets. Comment. Math. Helv. 51 (1976), no. $1,43-55$.

[CTZ] H. Christianson, J. A. Toth and S. Zelditch, Quantum ergodic restriction for Cauchy Data: Interior QUE and restricted QUE (arXiv:1205.0286).

[CM] T. H. Colding and W. P. Minicozzi II, Lower bounds for nodal sets of eigenfunctions. Comm. Math. Phys. 306 (2011), no. 3, 777 - 784.

[CV] Y.Colin de Verdière, Ergodicité et fonctions propres du Laplacien, Comm.Math.Phys. 102 (1985), 497-502.

[C] R. Cooper, The extremal values of Legendre polynomials and of certain related functions. Proc. Cambridge Philos. Soc. 46, (1950). 549-55.

[Dong] R-T. Dong, Nodal sets of eigenfunctions on Riemann surfaces. J. Differential Geom. 36 (1992), no. 2, 493-506.

[DF] H. Donnelly and C. Fefferman, Nodal sets of eigenfunctions on Riemannian manifolds, Invent. Math. 93 (1988), 161-183.

[DF2] H. Donnelly and C. Fefferman, Nodal sets of eigenfunctions: Riemannian manifolds with boundary. Analysis, et cetera, 251-262, Academic Press, Boston, MA, 1990 .

[DF3] H. Donnelly and C. Fefferman, Growth and geometry of eigenfunctions of the Laplacian. Analysis and partial differential equations, 635-655, Lecture Notes in Pure and Appl. Math., 122, Dekker, New York, 1990.

[DF4] H. Donnelly and C. Fefferman, Nodal sets for eigenfunctions of the Laplacian on surfaces. J. Amer. Math. Soc. 3 (1990), no. 2, 333-353.

[DSZ] M. R. Douglas, B. Shiffman, and S. Zelditch, Critical points and supersymmetric vacua. II. Asymptotics and extremal metrics. J. Differential Geom. 72 (2006), no. $3,381-427$.

[DZ] S. Dyatlov, and M. Zworski, Quantum ergodicity for restrictions to hypersurfaces (arXiv:1204.0284).

[EK] Y. Egorov and V. Kondratiev, On spectral theory of elliptic operators. Operator Theory: Advances and Applications, 89. Birkhäuser Verlag, Basel, 1996.

[EGJS] Y. Elon, S. Gnutzmann, C. Joas, and U. Smilansky, Geometric characterization of nodal domains: the area-to-perimeter ratio. J. Phys. A 40 (2007), no. 11, 26892707.

[EJN] A. Eremenko, D. Jakobson and N. Nadirashvili, On nodal sets and nodal domains on S2 and R2. Festival Yves Colin de Verdire. Ann. Inst. Fourier (Grenoble) 57 (2007), no. 7, 2345-2360.

[Fed] H. Federer, Geometric measure theory. Die Grundlehren der mathematischen Wissenschaften, Band 153 Springer-Verlag New York Inc., New York 1969.

[FGS] G. Foltin, S. Gnutzmann, and U. Smilansky, The morphology of nodal linesrandom waves versus percolation. J. Phys. A 37 (2004), no. 47, 11363-11371.

[GaL] N. Garofalo and F. H. Lin, Monotonicity properties of variational integrals, $A_{p}$ weights and unique continuation. Indiana Univ. Math. J. 35 (1986), no. 2, 245268.

[GaL2] — Unique continuation for elliptic operators: a geometric-variational approach. Comm. Pure Appl. Math. 40 (1987), no. 3, 347-366

[GS] I. M. Gelfand and M. Smirnov, Lagrangians satisfying Crofton formulas, Radon transforms, and nonlocal differentials. Adv. Math. 109 (1994), 188-227.

[GL] P.Gérard and E.Leichtnam, Ergodic properties of eigenfunctions for the Dirichlet problem, Duke Math J. 71 (1993), 559-607.

[Gi] V. M. Gichev, A Note on the Common Zeros of Laplace Beltrami Eigenfunctions. Ann. Global Anal. Geome. 26, 201-208 (2004). 
[GLS] F. Golse, E. Leichtnam, and M. Stenzel, Intrinsic microlocal analysis and inversion formulae for the heat equation on compact real-analytic Riemannian manifolds. Ann. Sci. École Norm. Sup. (4) 29 (1996), no. 6, 669-736.

[GSj] A. Grigis and J. Sjöstrand, Microlocal analysis for differential operators, London Math. Soc. Lecture Notes 196 (1994).

[GS1] V. Guillemin and M. Stenzel, Grauert tubes and the homogeneous Monge-Ampère equation. J. Differential Geom. 34 (1991), no. 2, 561-570.

[GS2] - Grauert tubes and the homogeneous Monge-Ampère equation. II. J. Differential Geom. 35 (1992), no. 3, 627-641.

[H2] Q. Han, Nodal sets of harmonic functions, Pure and Applied Mathematics Quarterly 3 (3) (2007), 647-688.

[HHL] Q. Han, R. Hardt, and F. H. Lin, Geometric measure of singular sets of elliptic equations. Comm. Pure Appl. Math. 51 (1998), no. 11-12, 1425-1443.

[H] Q. Han and F.H. Lin Nodal sets of solutions of elliptic differential equations, book in preparation (online at http://www.nd.edu/ qhan).

[HL] X. Han and G. Lu, A geometric covering lemma and nodal sets of eigenfunctions. (English summary) Math. Res. Lett. 18 (2011), no. 2, 337-352

[HHON] R. Hardt, M. Hoffmann-Ostenhof, T. Hoffmann-Ostenhof and N. Nadirashvili, Critical sets of solutions to elliptic equations. J. Differential Geom. 51 (1999), no. 2, 359-373.

[HaS] R. Hardt and L. Simon, Nodal sets for solutions of elliptic equations. J. Differential Geom. 30 (1989), no. 2, 505-522.

[HZ] A. Hassell and S. Zelditch, Quantum ergodicity of boundary values of eigenfunctions. Comm. Math. Phys. 248 (2004), no. 1, 119-168.

[Hel] S. Helgason, Topics in harmonic analysis on homogeneous spaces. Progress in Mathematics, 13. Birkhäuser, Boston, Mass., 1981.

[HEJ] E. J. Heller, Gallery (Quantum random waves), http://www.ericjhellergallery.com/.

[He] H. Hezari, Complex zeros of eigenfunctions of 1D Schrödinger operators. Int. Math. Res. Not. IMRN 2008, no. 3, Art. ID rnm148.

[HS] H. Hezari and C. D. Sogge, A natural lower bound for the size of nodal sets, Analysis and PDE vol. 5 (2012), 1133-1137 (arXiv:1107.3440).

[HW] H. Hezari and Z. Wang, Lower bounds for volumes of nodal sets: an improvement of a result of Sogge-Zelditch, to appear arXiv:1107.0092.

[HC] D. Hilbert and R. Courant, Methods of mathematical physics, Vol. I and Vol. II:. Interscience Publishers (John Wiley \& Sons), New York-Lon don 1962.

[HoI-IV] L. Hörmander, Theory of Linear Partial Differential Operators I-IV, SpringerVerlag, New York (1985).

[Hu] R. Hu, $L^{p}$ norm estimates of eigenfunctions restricted to submanifolds. Forum Math. 21 (2009), no. 6, 1021 - 1052.

[Isi] M. B. Isichenko, Percolation, statistical topography, and transport in random media. Rev. Modern Phys. 64 (1992), no. 4, 961-1043.

[IsiK] M. B. Isichenko and J. Kalda, Statistical topography. I. Fractal dimension of coastlines and number-area rule for islands. J. Nonlinear Sci. 1 (1991), no. 3, 255-277

[JN] D. Jakobson and N. Nadirashvili, Eigenfunctions with few critical points. J. Differential Geom. 53 (1999), no. 1, 177-182.

[JN2] - Quasi-symmetry of $L^{p}$ norms of eigenfunctions. Comm. Anal. Geom. 10 (2002), no. 2, 397-408.

[JM] D. Jakobson and D. Mangoubi, Tubular Neighborhoods of Nodal Sets and Diophantine Approximation, Amer. J. Math. 131 (2009), no. 4, 1109-1135 (arXiv:0707.4045). 
[JL] D. Jerison and G. Lebeau, Nodal sets of sums of eigenfunctions. Harmonic analysis and partial differential equations (Chicago, IL, 1996), 223-239, Chicago Lectures in Math., Univ. Chicago Press, Chicago, IL, 1999.

[JJ] J. Jung, Zeros of eigenfunctions on hyperbolic surfaces lying on a curve, to appear in JEMS (arXiv: 1108.2335).

[KH] J. Kondev and C. L. Henley, Geometrical exponents of contour loops on random Gaussian surfaces, Phys. Rev. Lett. 74 (1995), 4580 - 4583.

[KHS] J. Kondev, C. L. Henley, and D.G. Salinas, Nonlinear measures for characterizing rough surface morphologies. Phys. Rev. E, 61 (2000), 104-125.

[Kua] I. Kukavica, Nodal volumes for eigenfunctions of analytic regular elliptic problems. J. Anal. Math. 67 (1995), 269-280.

[Ku] , Quantitative uniqueness for second-order elliptic operators. Duke Math. J. 91 (1998), no. 2, 225-240.

[LS1] L. Lempert and R. Szöke, Global solutions of the homogeneous complex MongeAmpère equation and complex structures on the tangent bundle of Riemannian manifolds. Math. Ann. 290 (1991), no. 4, 689-712.

[LS2] The tangent bundle of an almost complex manifold, Canad. Math. Bull. 44 (2001), no. 1, 70-79.

[Lew] H. Lewy, On the minimum number of domains in which the nodal lines of spherical harmonics divide the sphere. Comm. Partial Differential Equations 2 (1977), no. $12,1233-1244$.

[Ley] J. Leydold, On the number of nodal domains of spherical harmonics. Topology 35 (1996), no. 2, 301-321.

[Lin] F.H. Lin, Nodal sets of solutions of elliptic and parabolic equations. Comm. Pure Appl. Math. 44 (1991), no. 3, 287-308.

[Man] D. Mangoubi, A Remark on Recent Lower Bounds for Nodal Sets, Comm. Partial Differential Equations 36 (2011), no. 12, 2208-2212 (arXiv:1010.4579.)

[Man2] D. Mangoubi, The Volume of a Local Nodal Domain, J. Topol. Anal. 2 (2010), no. 2, 259-275 ( arXiv:0806.3327).

[Man3] D. Mangoubi, On the inner radius of a nodal domain. Canad. Math. Bull. 51 (2008), no. 2, 249-260.

[Me] A. D. Melas, On the nodal line of the second eigenfunction of the Laplacian in $\mathbf{R}^{2}$ R2. J. Differential Geom. 35 (1992), no. 1, 255-263.

[Mi] J. Miller, Universality for SLE(4), arXiv:1010.1356.

[NJT] N. Nadirashvili, D. Jakobson, and J.A. Toth, Geometric properties of eigenfunctions. (Russian) Uspekhi Mat. Nauk 56 (2001), no. 6(342), 67-88; translation in Russian Math. Surveys 56 (2001), no. 6, 1085-1105

[NPS] F. Nazarov, L. Polterovich and M. Sodin, Sign and area in nodal geometry of Laplace eigenfunctions. Amer. J. Math. 127 (2005), no. 4, 879-910.

[NS] F. Nazarov and M. Sodin, On the Number of Nodal Domains of Random Spherical Harmonics. Amer. J. Math. 131 (2009), no. 5, 1337-1357 (arXiv:0706.2409).

[Neu] J. Neuheisel, "Asymptotic distribution of nodal sets on spheres," PhD Thesis, Johns Hopkins University, Baltimore, MD 2000, 1994, http://mathnt.mat.jhu.edu/mathnew/Thesis/joshuaneuheisel.pdf.

[Nic] L. I. Nicolaescu, Critical sets of random smooth functions on compact manifolds (arXiv:1008.5085).

[P] A. Pleijel, Remarks on Courant's nodal line theorem, Comm. Pure Appl. Math., 9, 543-550 (1956).

[Po] I. Polterovich, Pleijel's nodal domain theorem for free membranes, Proc. Amer. Math. Soc. 137 (2009), no. 3, 1021-1024 (arXiv:0805.1553).

[PS] L. Polterovich and M. Sodin, Nodal inequalities on surfaces. Math. Proc. Cambridge Philos. Soc. 143 (2007), no. 2, 459-467 (arXiv:math/0604493). 
[R] J. Ralston, Gaussian beams and the propagation of singularities. Studies in partial differential equations, 206-248, MAA Stud. Math., 23, Math. Assoc. America, Washington, DC, 1982.

[Reu] M. Reuter. Hierarchical Shape Segmentation and Registration via Topological Features of Laplace-Beltrami Eigenfunctions. International Journal of Computer Vision 89 (2), pp. 287-308, 2010.

[Reu2] M. Reuter, Laplace Spectra for Shape Recognition, Books on Demand (2006).

[Ri] G. Rivière, Letter to the author (2012).

[SY] R. Schoen and S. T. Yau, Lectures on differential geometry. . Conference Proceedings and Lecture Notes in Geometry and Topology, I. International Press, Cambridge, MA, 1994.

[Sh.1] A.I.Shnirelman, Ergodic properties of eigenfunctions, Usp.Math.Nauk. 29/6 (1974), 181-182.

[SS] U. Smilansky and H.-J. Stöckmann, Nodal Patterns in Physics and Mathematics, The European Physical Journal Special Topics Vol. 145 (June 2007).

[Sog] C. D. Sogge, Concerning the $L^{p}$ norm of spectral clusters for second-order elliptic operators on compact manifolds, J. Funct. Anal. 77 (1988), 123-138.

[Sog2] C. D. Sogge, Kakeya-Nikodym averages and $L^{p}$-norms of eigenfunctions, Tohoku Math. J. (2) 63 (2011), no. 4, 519-538 (arXiv:0907.4827).

[Sog3] C. D. Sogge: Fourier integrals in classical analysis, Cambridge Tracts in Mathematics, 105, Cambridge University Press, Cambridge, 1993.

[STZ] C.D. Sogge, J. A. Toth and S. Zelditch, About the blowup of quasimodes on Riemannian manifolds. J. Geom. Anal. 21 (2011), no. 1, 150-173.

[SoZ] C. D. Sogge and S. Zelditch, Lower bounds on the hypersurface measure of nodal sets, Math. Research Letters 18 (2011), 27-39 (arXiv:1009.3573).

[SoZa] C. D. Sogge and S. Zelditch, Lower bounds on the Hausdorff measure of nodal sets II, to appear in Math. Res. Lett. (arXiv:1208.2045).

[SoZ2] C.D. Sogge and S. Zelditch, On eigenfunction restriction estimates and $L^{4}$-bounds for compact surfaces with nonpositive curvature (arXiv:1108.2726).

[SoZ3] C.D. Sogge and S. Zelditch, Concerning the $L^{4}$ norms of typical eigenfunctions on compact surfaces, Recent Developments in Geometry and Analysis, 23 (2013), 407-423, International Press of Boston, Boston (arXiv:1011.0215).

[SA] D. Stauffer and A. Aharony, Introduction to Percolation theory, Taylor and Francis, London (1994).

[Sz] G. Szegö, Inequalities for the zeros of Legendre polynomials and related functions. Trans. Amer. Math. Soc. 39 (1936), no. 1, 1-17.

[Sz2] G. Szegö. On the relative extrema of Legendre polynomials. Boll. Un. Mat. Ital. (3) $5,(1950)$. 120-121.

[Taa] D. Tataru, On the regularity of boundary traces for the wave equation, Ann. Scuola Norm. Sup. Pisa Cl. Sci. (4) 26 (1998), 185 - 206.

[TZ] J. A. Toth and S. Zelditch, Counting Nodal Lines Which Touch the Boundary of an Analytic Domain, Jour. Diff. Geom. 81 (2009), 649- 686 (arXiv:0710.0101).

[TZ2] J. A. Toth and S. Zelditch, Quantum ergodic restriction theorems, I: interior hypersurfaces in analytic domains,, Ann. H. Poincaré 13, Issue 4 (2012), Page 599-670 (arXiv:1005.1636).

[TZ3] J. A. Toth and S. Zelditch, Quantum ergodic restriction theorems, II: manifolds without boundary, to appear in GAFA (arXiv:1104.4531).

[U] K. Uhlenbeck, Generic properties of eigenfunctions. Amer. J. Math. 98 (1976), no. 4, 1059-1078.

[Web] J. Weber, The Morse-Witten complex via dynamical systems. Expo. Math. 24 (2006), no. 2, 127-159.

[Wei] A. Weinrib, Percolation threshold of a two-dimensional continuum system. Phys. Rev. B (3) 26 (1982), no. 3, 1352-1361. 
[Wig] I. Wigman, On the distribution of the nodal sets of random spherical harmonics. J. Math. Phys. 50 (2009), no. 1, 013521.

[Wu $\quad$ F. Y. Wu, Percolation and the Potts model. J. Statist. Phys. 18 (1978), no. 2, $115-123$

[Y1] S.T. Yau, Survey on partial differential equations in differential geometry. Seminar on Differential Geometry, pp. 3-71, Ann. of Math. Stud., 102, Princeton Univ. Press, Princeton, N.J., 1982.

[Y2] - Open problems in geometry. Differential geometry: partial differential equations on manifolds (Los Angeles, CA, 1990), 1-28, Proc. Sympos. Pure Math., 54, Part 1, Amer. Math. Soc., Providence, RI, 1993.

[Y3] - A note on the distribution of critical points of eigenfunctions, Tsing Hua Lectures in Geometry and Analysis 315-317, Internat. Press, 1997.

[Z1] S. Zelditch, Uniform distribution of eigenfunctions on compact hyperbolic surfaces. Duke Math. J. 55 (1987), no. 4, 919-941

[Z2] S. Zelditch, Complex zeros of real ergodic eigenfunctions. Invent. Math. 167 (2007), no. 2, 419-443.

[Z3] S. Zelditch, Ergodicity and intersections of nodal sets and eigenfunctions on real analytic surfaces (arXiv:1210.0834).

[Z4] S. Zelditch, Real and complex zeros of Riemannian random waves. Spectral analysis in geometry and number theory, 321-342, Contemp. Math., 484, Amer. Math. Soc., Providence, RI, 2009.

[Z5] S. Zelditch, Local and global analysis of eigenfunctions on Riemannian manifolds. Handbook of geometric analysis. No. 1, 545-658, Adv. Lect. Math. (ALM), 7, Int. Press, Somerville, MA, 2008.

[Z6] S. Zelditch, New Results in Mathematics of Quantum Chaos, Current Developments in Mathematics 2009, p. 115- 202 (arXiv:0911.4312).

[Z7] S. Zelditch, Kuznecov sum formulae and Szego limit formulae on manifolds, Comm. PDE 17 (1\&2) (1992), 221-260.

[Z8] S. Zelditch, Pluri-potential theory on Grauert tubes of real analytic Riemannian manifolds, I. Spectral geometry, 299 - 339, Proc. Sympos. Pure Math., 84, Amer. Math. Soc., Providence, RI, 2012.

[Z9] S. Zelditch, Complex zeros of quantum integrable eigenfunctions, (in preparation).

[ZZw] S.Zelditch and M.Zworski, Ergodicity of eigenfunctions for ergodic billiards, Comm.Math. Phys. 175 (1996), 673-682.

[Zw] M. Zworski, Semiclassical analysis, Graduate Studies in Mathematics, 138. American Mathematical Society, Providence, RI, 2012.

Department of Mathematics, Northwestern University, Evanston, IL 60208, USA

E-mail address: zelditch@math.northwestern.edu 